CONFORMAL GEOMETRY AND DYNAMICS

An Electronic Journal of the American Mathematical Society

Volume 15, Pages 72-112 (August 1, 2011)

S 1088-4173(2011)00228-1

\title{
ITÉRATION D'APPLICATIONS RATIONNELLES DANS LES ESPACES DE MATRICES
}

\author{
DOMINIQUE CERVEAU AND JULIE DÉSERTI
}

\begin{abstract}
The iteration of rational maps is well understood in dimension 1 but less so in higher dimensions. We study some maps on spaces of matrices which present a weak complexity with respect to the ring structure. First, we give some properties of certain rational maps; the simplest example is the rational map which sends the matrix $\mathrm{M}$ onto $\mathrm{M}^{2}$ for which we exhibit some dynamical properties. Finally, we deal with some small perturbations of this map.
\end{abstract}

\section{INTRODUCTION}

L'itération des applications rationnelles est très bien comprise en dimension un, un peu moins en dimension deux et encore moins en dimension plus grande. Nous nous proposons d'étudier ici des applications spéciales sur les espaces de matrices qui présentent une « faible complexité » par rapport à la structure d'anneau. Par souci de simplicité nous travaillons sur les matrices $2 \times 2$, bien que la plupart du discours se laisse généraliser sans problème.

Dans un premier temps nous nous intéressons aux transformations compatibles à la conjugaison, i.e. aux applications rationnelles $\Phi: \mathcal{M}(2 ; \mathbb{C}) \rightarrow \mathcal{M}(2 ; \mathbb{C})$ telles que $\mathrm{A} \Phi(\mathrm{M}) \mathrm{A}^{-1}=\Phi\left(\mathrm{AMA}^{-1}\right)$ pour tout $\mathrm{A}$ dans $\mathrm{GL}(2 ; \mathbb{C})$ et tout $\mathrm{M}$ là où cela a un sens. Un exemple de ce type d'applications est donné par les polynômes de matrices. Nous commençons par présenter des propriétés satisfaites par ces transformations comme par exemple : l'invariance du groupe diagonal $\mathcal{D}$ ou encore le fait qu'une telle transformation est birationnelle si et seulement si sa restriction à $\mathcal{D}$ l'est. Au centre $\mathcal{C}=\{\lambda \mathrm{Id} \mid \lambda \in \mathbb{C}\}$ on peut associer la fibration $\mathcal{P}$ en 2-plans définie comme suit : si $\mathrm{M}$ désigne un élément de $\mathcal{M}(2 ; \mathbb{C}) \backslash \mathcal{C}$, on définit $\mathcal{P}(\mathrm{M})$ comme l'unique plan contenant $\mathrm{M}$ et $\mathcal{C}$ : le 2 -plan $\mathcal{P}(\mathrm{M})$ n'est rien d'autre que l'ensemble des matrices qui commutent à $\mathrm{M}$. Cette fibration est invariante fibre à fibre par toute application rationnelle compatible à la conjugaison. Bien sûr on peut considérer l'application Inv de $\mathcal{M}(2 ; \mathbb{C})$ dans $\mathbb{C}^{2}$ qui à une matrice $\mathrm{M}$ associe ses invariants de similitude (tr M, det M). Par définition une application rationnelle $\Phi$ compatible à la conjugaison laisse invariant le feuilletage associé à la fibration Inv ; plus précisément il existe une application rationnelle Sq $\Phi: \mathbb{C}^{2} \rightarrow \mathbb{C}^{2}$ telle que Inv $\odot \Phi=\operatorname{Sq} \Phi \circ$ Inv. Réciproquement on peut se demander à quelle condition une application rationnelle de $\mathbb{C}^{2}$ se relève à $\mathcal{M}(2 ; \mathbb{C})$, question à laquelle nous répondrons. Évidemment la

Received by the editors March 7, 2011.

2010 Mathematics Subject Classification. Primary 14E05, 32H50, 37 B05.

(C)2011 American Mathematical Society 
transformation $\mathrm{Sq} \Phi$ contient une grande partie de la dynamique de l'application initiale $\Phi$.

Nous nous intéressons ensuite tout particulièrement à l'application $\Phi_{\mathrm{Id}}: \mathcal{M}(2 ; \mathbb{C})$ $\rightarrow \mathcal{M}(2 ; \mathbb{C}), \mathrm{M} \mapsto \mathrm{M}^{2}$, exemple typique d'application compatible à la conjugaison. Après avoir donné quelques propriétés satisfaites par cette application, nous décrivons l'adhérence de ses points périodiques ainsi que le bord du bassin d'attraction de la matrice nulle qui donne naissance à une intéressante hypersurface LEVI-plate. Une façon de mesurer la complexité d'une transformation est d'examiner son centralisateur. C'est dans cette optique que nous déterminons le groupe $\operatorname{Aut}\left(\mathcal{M}(2 ; \mathbb{C}) ; \Phi_{\text {Id }}\right)$ des automorphismes holomorphes de $\mathcal{M}(2 ; \mathbb{C})$ qui commutent à $\Phi_{\text {Id }}$ ainsi que $\operatorname{Bir}\left(\mathcal{M}(2 ; \mathbb{C}) ; \Phi_{\text {Id }}\right)$ le groupe des transformations birationnelles de $\mathcal{M}(2 ; \mathbb{C})$ qui commutent à $\Phi_{\text {Id }} ;$ le premier est engendré par les applications de conjugaison $\sigma_{\mathrm{P}}: \mathrm{M} \mapsto \mathrm{PMP}^{-1}$ où $\mathrm{P}$ appartient à $\mathrm{GL}(2 ; \mathbb{C})$, et la transposition $\mathrm{M} \mapsto{ }^{\mathrm{t}} \mathrm{M}$. Pour obtenir le second il faut ajouter l'application «inverse $\gg: \mathrm{M} \mapsto \mathrm{M}^{-1}$.

Proposition 1. Le groupe $\operatorname{Aut}\left(\mathcal{M}(2 ; \mathbb{C}) ; \Phi_{\text {Id }}\right)$ est isomorphe à $\operatorname{PGL}(2 ; \mathbb{C}) \rtimes \mathbb{Z} / 2 \mathbb{Z}$.

Le groupe $\operatorname{Bir}\left(\mathcal{M}(2 ; \mathbb{C}) ; \Phi_{\text {Id }}\right)$ est engendré par $\operatorname{Aut}\left(\mathcal{M}(2 ; \mathbb{C}) ; \Phi_{\text {Id }}\right)$ et par l'involution $\iota: \mathrm{M} \mapsto \mathrm{M}^{-1}$.

Enfin nous considérons des déformations spéciales de $\Phi_{\mathrm{Id}}, \Phi_{\mathrm{A}}: \mathcal{M}(2 ; \mathbb{C}) \rightarrow$ $\mathcal{M}(2 ; \mathbb{C}), \mathrm{M} \mapsto \mathrm{AM}^{2}$ avec $\mathrm{A}$ dans $\mathrm{GL}(2 ; \mathbb{C})$. Ce sont les applications $\ll$ monomiales $\gg$ les plus simples et elles sont en général non compatibles à la conjugaison. Alors qu'à une variable les transformations $z \mapsto z^{2}$ et $z \mapsto a z^{2}$ sont linéairement conjuguées, la situation ici est plus complexe. Nous précisons tout du moins pour A générique, i.e. pour A de la forme $\left[\begin{array}{cc}\lambda & 0 \\ 0 & \frac{1}{\lambda}\end{array}\right]$, l'ensemble des orbites périodiques et l'adhérence de cet ensemble :

Proposition 2. Pour $\lambda$ générique l'adhérence des points périodiques de $\Phi_{\mathrm{A}}$ est constituée

- d'un tore $\mathbb{S}^{1} \times \mathbb{S}^{1}$ contenu dans $y=z=0$;

- de deux $\mathbb{S}^{1} \times \mathbb{C}$, précisément $\frac{1}{\lambda} \mathbb{S}^{1} \times \mathbb{C} \times\{0\} \times\{0\}$ et $\{0\} \times\{0\} \times \mathbb{C} \times \lambda \mathbb{S}^{1}($ en identifiant $\left.\mathcal{M}(2 ; \mathbb{C})=\left\{\left[\begin{array}{cc}x & y \\ z & t\end{array}\right]\right\} \grave{a} \mathbb{C}^{4}=\{(x, y, z, t) \mid x, y, z, t \in \mathbb{C}\}\right) ;$

- de la matrice nulle.

Alors que pour $z \mapsto z^{2}$ l'adhérence des points périodiques (différent de 0 ) est le bord du bassin d'attraction de l'origine, nous déduisons de la Proposition 2 que ce n'est pas le cas ici. Nous donnons aussi des exemples d'orbites bornées, non périodiques et non contenues dans le bassin d'attraction de l'origine. Le cas où $\mathrm{A}$ est une matrice quaternionique est particulièrement riche. Nous effectuons quelques expériences numériques afin de mieux comprendre le bassin d'attraction de la matrice nulle, son bord ainsi que la dynamique de ce type de transformations. En particulier nous nous intéressons à la restriction de $\Phi_{\mathrm{A}}$ à l'ensemble $\mathbb{H}_{1}$ des quaternions de module 1 qui est décrite par $f_{\vartheta}:(x, y) \mapsto\left(\mathrm{e}^{\mathrm{i} \vartheta}\left(x^{2}+|x|^{2}-1\right), \mathrm{e}^{\mathrm{i} \vartheta} y(x+\bar{x})\right)$; remarquons qu'elle laisse la famille de cercles paramétrée par $\eta \mapsto\left(x, y \mathrm{e}^{\mathrm{i} \eta}\right)$ globalement invariante. La première composante $f_{\vartheta}^{1}$ de $f_{\vartheta}$ indique comment passer d'un cercle à l'autre; elle s'identifie à une application de $\mathbb{R}^{2}$ dans lui-même ce qui nous permet de déterminer quelques-unes de ses propriétés. Les applications $f_{\vartheta}^{1}$ préservent le disque unité de $\mathbb{R}^{2}$ et c'est la dynamique dans ce disque qui nous 
intéresse. Nous avons par exemple réalisé des expériences numériques visant à mesurer la façon dont les orbites s'approchent du bord du disque unité et à quelle vitesse :

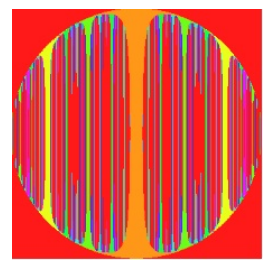

$\vartheta=0$

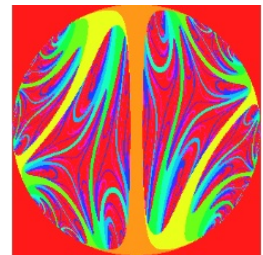

$\vartheta=1$

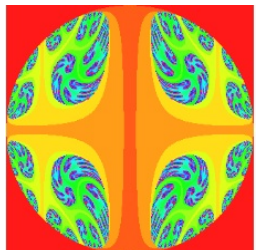

$\vartheta=\frac{\pi}{2}$

Comme dans le cas spécial où $\mathrm{A}=\mathrm{Id}$ nous déterminons le sous-groupe des transformations biholomorphes de $\mathcal{M}(2 ; \mathbb{C})$ qui commutent à $\Phi_{\mathrm{A}}$.

Proposition 3. Si A est un multiple de l'identité alors $\Phi_{\mathrm{A}}$ est conjugué à $\Phi_{\mathrm{Id}}$, sinon $\operatorname{Aut}\left(\mathcal{M}(2 ; \mathbb{C}) ; \Phi_{\mathrm{A}}\right)$ est formé des $\sigma_{\mathrm{P}}$ où $\sigma_{\mathrm{P}}$ désigne l'application $\mathrm{M} \mapsto \mathrm{PMP}^{-1}$ et $\mathrm{P}$ une matrice qui commute à $\mathrm{A}$. En fait $\operatorname{Aut}\left(\mathcal{M}(2 ; \mathbb{C}) ; \Phi_{\mathrm{A}}\right)$ s'identifie à $\mathbb{C}^{*}$ agissant sur $\mathcal{M}(2 ; \mathbb{C})$ de la façon suivante $:(x, y, z, t, \alpha) \mapsto\left(x, \alpha y, \frac{z}{\alpha}, t\right)$. Les orbites de cette action sont aussi celles du champ de vecteurs invariant $y \frac{\partial}{\partial y}-z \frac{\partial}{\partial z}$.

Remarquons que le bord du bassin d'attraction de l'origine suivant $\Phi_{\mathrm{A}}$ est invariant par l'action de ce groupe. Beaucoup de questions concernant ces transformations $\Phi_{\mathrm{A}}$ restent ouvertes; nous listons celles qui nous paraissent les plus pertinentes, tant sur le plan théorique que d'un point de vue numérique.

\section{GÉNÉRALitÉS}

Rappelons quelques définitions.

Définitions. Une application méromorphe $f: X \rightarrow Z$ entre deux variétés complexes compactes est définie par son graphe $\Gamma(f) \subset X \times Z$; ce graphe est une sous-variété irréductible pour laquelle la projection $\pi_{1}: \Gamma(f) \rightarrow X$ sur le premier facteur est une application holomorphe surjective propre dont la fibre générique est un point ([5]). Le lieu d'indétermination de $f$ est l'ensemble des points où $\pi_{1}$ n'admet pas d'inverse local, on le note Ind $f$. L'application $f$ est dominante si la seconde projection $\pi_{2}: \Gamma(f) \rightarrow Z$ est surjective. Notons Exc $\pi_{2}$ l'ensemble des points où $\pi_{2}$ n'est pas une application finie; on définit l'ensemble exceptionnel de $f$ par $\operatorname{Exc} f=\pi_{1}\left(\operatorname{Exc} \pi_{2}\right)$.

Dans le cas particulier où $f: \mathbb{C}^{n+1} \rightarrow \mathbb{C}^{n+1}$ est une application polynomiale homogène ad-hoc représentant la transformation rationnelle

$$
\mathbb{P}(f): \mathbb{P}^{n}(\mathbb{C}) \rightarrow \mathbb{P}^{n}(\mathbb{C}),
$$

un point $[m]=\left(m_{0}: \ldots: m_{n}\right)$ est un point d'indétermination de $\mathbb{P}(f)$ si $f(m)=0$. Une sous-variété irréductible $V \subset \mathbb{P}^{n}(\mathbb{C})$ est contractée par $\mathbb{P}(f)$ si la dimension de $\overline{\mathbb{P}(f)(V \backslash \text { Ind } \mathbb{P}(f))}$ est strictement inférieure à celle de $V$. Une telle sous-variété est contenue dans l'ensemble $\operatorname{Exc} \mathbb{P}(f)$.

Une application homogène $f: \mathbb{C}^{n+1} \rightarrow \mathbb{C}^{n+1}$ respecte la fibration de Hopf par les droites passant par l'origine : une droite $d$ est envoyée par $f$ sur une autre droite $f(d)$ à moins que $f$ s'annule sur $d$. L'application $\mathbb{P}(f)$ explique comment sont échangées ces droites par $f$. 
Nous travaillerons aussi avec des applications rationnelles $f: \mathbb{C}^{n} \rightarrow \mathbb{C}^{n}$ qui sont simplement les applications dont les composantes $f_{i}$ sont rationnelles, i.e. des quotients de polynômes. Pour ces applications on a aussi la notion de sous-variétés contractées et d'ensemble d'indétermination qui cö̈ncide avec l'union des pôles des composantes.

Soit $\mathcal{M}(2 ; \mathbb{C})=\left\{\left[\begin{array}{ll}a & b \\ c & d\end{array}\right] \mid a, b, c, d \in \mathbb{C}\right\} \simeq \mathbb{C}^{4}$ l'ensemble des matrices $2 \times 2$ à coefficients complexes. Notons Inv l'application de $\mathcal{M}(2 ; \mathbb{C})$ à valeurs dans $\mathbb{C}^{2}$ qui à une matrice associe ses invariants de similitude

$$
\text { Inv : } \mathcal{M}(2 ; \mathbb{C}) \rightarrow \mathbb{C}^{2}, \quad \mathrm{M} \mapsto(\operatorname{tr} \mathrm{M}, \operatorname{det} \mathrm{M})
$$

avec les notations habituelles. La sous-algèbre de $\mathcal{M}(2 ; \mathbb{C})$ des matrices diagonales sera notée $\mathcal{D}$

$$
\mathcal{D}=\left\{\left[\begin{array}{cc}
\lambda_{1} & 0 \\
0 & \lambda_{2}
\end{array}\right] \mid \lambda_{i} \in \mathbb{C}\right\}
$$

Désignons par $\operatorname{diag}\left(\lambda_{1}, \lambda_{2}\right)$ l'élément $\left[\begin{array}{cc}\lambda_{1} & 0 \\ 0 & \lambda_{2}\end{array}\right]$ de $\mathcal{D}$ et par $\mathbf{0}$ la matrice nulle $\operatorname{diag}(0,0)$.

Soit $\Phi: \mathcal{M}(2 ; \mathbb{C}) \rightarrow \mathcal{M}(2 ; \mathbb{C})$ une application rationnelle dominante. Si l'on identifie $\mathcal{M}(2 ; \mathbb{C})$ à $\mathbb{C}^{4}$ que l'on voit comme carte affine de $\mathbb{P}^{4}(\mathbb{C})$, alors $\Phi$ induit une application rationnelle notée $\widetilde{\Phi}$ de $\mathbb{P}^{4}(\mathbb{C})$ dans lui-même. Dans le cas spécial où $\Phi$ est homogène $\left(\Phi(s \mathrm{M})=s^{d} \Phi(\mathrm{M})\right), \Phi$ induit une application rationnelle notée $\mathbb{P}(\Phi): \mathbb{P}^{3}(\mathbb{C}) \rightarrow \mathbb{P}^{3}(\mathbb{C})$.

Définition. Une application $\Phi: \mathcal{M}(2 ; \mathbb{C}) \rightarrow \mathcal{M}(2 ; \mathbb{C})$ sera dite monomiale (relativement à la multiplication) si elle est du type

$$
\Phi(\mathrm{M})=\mathrm{A}_{1} \mathrm{MA}_{2} \mathrm{M} \ldots \mathrm{A}_{p} \mathrm{MA}_{p+1}
$$

où les $\mathrm{A}_{i}$ sont des éléments de $\mathrm{GL}(2 ; \mathbb{C})$ fixés.

Exemple 1.1. L'application $\Phi_{\mathrm{Id}}: \mathcal{M}(2 ; \mathbb{C}) \rightarrow \mathcal{M}(2 ; \mathbb{C})$ définie par $\Phi_{\mathrm{Id}}(\mathrm{M})=\mathrm{M}^{2}$ est monomiale. Un calcul élémentaire montre que si $\mathrm{M}=\left[\begin{array}{cc}x & y \\ z & t\end{array}\right]$, alors $\Phi_{\mathrm{Id}}(\mathrm{M})=$ $\left[\begin{array}{cc}x^{2}+y z & y(x+t) \\ z(x+t) & t^{2}+y z\end{array}\right]$. L'application $\Phi_{\mathrm{Id}}$ est génériquement finie au sens suivant : pour $\mathrm{M}$ générique, $\# \Phi_{\mathrm{Id}}^{-1}(\mathrm{M})=4$. Si M est une matrice nilpotente, i.e. $\operatorname{Inv}(\mathrm{M})=$ $(0,0)$, alors $\Phi_{\text {Id }}(M)=\mathbf{0}$ de sorte que $[\mathrm{M}]$ est un point d'indétermination de $\mathbb{P}\left(\Phi_{\mathrm{Id}}\right)$. En fait l'ensemble $\operatorname{Ind}\left(\mathbb{P}\left(\Phi_{\mathrm{Id}}\right)\right)$ des points d'indétermination de $\mathbb{P}\left(\Phi_{\mathrm{Id}}\right)$ est exactement le projectivisé de l'ensemble $\mathcal{N}(2 ; \mathbb{C})=\{\mathrm{M} \in \mathcal{M}(2 ; \mathbb{C}) \mid \operatorname{Inv}(\mathrm{M})=(0,0)\}$ des matrices nilpotentes; c'est donc une conique plane lisse. Remarquons que l'image de $\Phi_{\text {Id }}$ est précisément

$$
W=\{\mathbf{0}\} \cup(\mathcal{M}(2 ; \mathbb{C}) \backslash \mathcal{N}(2 ; \mathbb{C})) .
$$

La sous-algèbre $\mathfrak{s l}(2 ; \mathbb{C})=\{\mathrm{M} \in \mathcal{M}(2 ; \mathbb{C}) \mid \operatorname{tr} \mathrm{M}=0\}$ est contractée par $\Phi_{\text {Id }}$ sur le centre $\mathcal{C}=\{\lambda \operatorname{Id} \mid \lambda \in \mathbb{C}\}$.

Remarquons que les matrices triangulaires supérieures forment un sous-espace invariant par $\Phi_{\mathrm{Id}}$, de même que les matrices triangulaires inférieures, et bien sûr $\mathcal{D}$, qui est leur intersection. Leurs projectivisés sont invariants par $\mathbb{P}\left(\Phi_{\text {Id }}\right)$. De la même 
façon la quadrique $\mathcal{Q}=\{\mathrm{M} \in \mathcal{M}(2 ; \mathbb{C}) \mid$ det $\mathrm{M}=0\}$ est complètement invariante par $\Phi_{\text {Id }}$. On peut aisément vérifier que le déterminant jacobien de $\Phi_{\text {Id }}$ est

$$
\operatorname{det} j a c \Phi_{\operatorname{Id}_{(\mathrm{M})}}=4(\operatorname{tr} \mathrm{M})^{2} \operatorname{det} \mathrm{M} \text {; }
$$

de plus, lorsque det $\mathrm{M}=0$ nous avons $\Phi_{\mathrm{Id}}(\mathrm{M})=(\operatorname{tr} \mathrm{M}) \mathrm{M}$. En résulte que le projectivisé des matrices de trace nulle $\mathbb{P}(\{\mathrm{M} \in \mathcal{M}(2 ; \mathbb{C}) \mid \operatorname{tr} \mathrm{M}=0\})$ est la seule surface de $\mathbb{P}^{3}(\mathbb{C})$ contractée par $\mathbb{P}\left(\Phi_{\mathrm{Id}}\right)$. Nous reviendrons plus tard (§3) sur la dynamique de l'application $\Phi_{\text {Id }}$ qui se réduit peu ou prou à celle de $z \mapsto z^{2}$ dans le plan complexe (ne serait-ce que via la formule $\operatorname{det} \mathrm{M}^{2}=(\operatorname{det} \mathrm{M})^{2}$ qui induit une «semi-conjugaison $\gg)$ mais produit des objets intéressants.

Définition. Soit $\Phi: \mathcal{M}(2 ; \mathbb{C}) \rightarrow \mathcal{M}(2 ; \mathbb{C})$ une application rationnelle. On dit que $\Phi$ est compatible à la conjugaison si

$$
\mathrm{A} \Phi(\mathrm{M}) \mathrm{A}^{-1}=\Phi\left(\mathrm{AMA}^{-1}\right)
$$

chaque fois que $\mathrm{M}$ et $\mathrm{AMA}^{-1}$ sont dans $\mathcal{M}(2 ; \mathbb{C}) \backslash \operatorname{Ind} \Phi$.

Notons que si $\Phi$ est compatible à la conjugaison, alors Ind $\Phi$ est invariant sous l'action adjointe $\mathrm{AMA}^{-1}$.

Ainsi l'application $\Phi_{\mathrm{Id}}$ définie précédemment est compatible à la conjugaison ; il en est de même des polynômes d'endomorphismes. Plus généralement si $r(s)=$ $P(s) / Q(s)$ est une fonction rationnelle en une variable $s$, alors $r(\mathrm{M})=P(\mathrm{M}) Q(\mathrm{M})^{-1}$ définit une application rationnelle compatible à la conjugaison. Une application compatible à la conjugaison induit une application de l'espace quotient $\mathcal{M}(2 ; \mathbb{C})$ /conjugaison. Tout ceci se généralise évidemment en dimension quelconque.

Remarque 1.2. Une application monomiale compatible à la conjugaison est du type $\Phi_{\alpha, d}(\mathrm{M})=\alpha \mathrm{M}^{d}, \alpha$ dans $\mathbb{C}^{*}$. L'algèbre $\mathfrak{s l}(2 ; \mathbb{C})$ n'est pas toujours contractée par $\Phi_{\alpha, d}$; par exemple pour $\mathrm{M}=\left[\begin{array}{cc}x & y \\ z & -x\end{array}\right]$ et $d=3$ nous avons

$$
\mathrm{M}^{3}=\left(x^{2}+y z\right)\left[\begin{array}{cc}
x & y \\
z & -x
\end{array}\right] .
$$

Dans ce cas les matrices de trace nulle sont fixes pour l'application $\mathbb{P}\left(\Phi_{\alpha, 3}\right)$ induite sur $\mathbb{P}^{3}(\mathbb{C})=\mathbb{P}(\mathcal{M}(2 ; \mathbb{C}))$. On constate que l'ensemble d'indétermination de $\mathbb{P}\left(\Phi_{\alpha, d}\right)$ est encore le projectivisé de $\mathcal{N}(2 ; \mathbb{C})$. Plus généralement nous avons en restriction à $\mathfrak{s l}(2 ; \mathbb{C})$,

$$
\mathrm{M}^{2 d}=\left(x^{2}+y z\right)^{d} \mathrm{Id}, \quad \quad \mathrm{M}^{2 d+1}=\left(x^{2}+y z\right)^{d} \mathrm{M} .
$$

Remarque 1.3. Soit $\Phi$ l'application monomiale définie par $\Phi(\mathrm{M})=\mathrm{A}_{1} \mathrm{M} \ldots$ $\mathrm{A}_{n} \mathrm{MA}_{n+1}, n \geq 2$. Quitte à conjuguer $\Phi$ par un automorphisme de $\mathcal{M}(2 ; \mathbb{C}) \mathrm{du}$ type $\mathrm{M} \mapsto \mathrm{PMQ}$ on peut supposer que $\mathrm{A}_{n}=\mathrm{Id}$. On en déduit après cette conjugaison que l'ensemble $\mathcal{N}(2 ; \mathbb{C})$ des matrices nilpotentes est aussi contracté sur $\mathbf{0}$.

À conjugaison près par $\mathrm{M} \mapsto \rho \mathrm{M}$ on peut se ramener à $\operatorname{det} \Phi(\mathrm{M})=(\operatorname{det} \mathrm{M})^{n}$. En particulier les hypersurfaces quadratiques $\{M \in \mathcal{M}(2 ; \mathbb{C}) \mid \operatorname{det} M=\varepsilon\}$ sont invariantes par l'itéré $\Phi^{p}$ pour chaque racine $\left(n^{p}-1\right)$-ième de l'unité. Évidemment les quadriques $\{\mathrm{M} \in \mathcal{M}(2 ; \mathbb{C}) \mid \operatorname{det} \mathrm{M}=0\}$ et $\mathrm{SL}(2 ; \mathbb{C})=\{\mathrm{M} \in \mathcal{M}(2 ; \mathbb{C}) \mid \operatorname{det} \mathrm{M}=$ $1\}$ sont invariantes par $\Phi$. 


\section{Applications COMpatibles À la CONJUGaison, PropriétéS Et EXEMPles}

2.1. Premières propriétés. Il est à peu près clair qu'une application compatible à la conjugaison est déterminée par sa restriction aux matrices diagonales. Précisons celà.

Lemme 2.1. Soit $\Phi: \mathcal{M}(2 ; \mathbb{C}) \rightarrow \mathcal{M}(2 ; \mathbb{C})$ une application rationnelle compatible à la conjugaison. Alors $\mathcal{D}$ est invariant par $\Phi$, i.e. $\Phi(\mathcal{D} \backslash \operatorname{Ind} \Phi) \subset \mathcal{D}$.

Démonstration. Remarquons que, par densité des matrices diagonalisables, $\mathcal{D}$ n'est pas contenu dans Ind $\Phi$. Soit $\mathrm{M}$ un élément de $\mathcal{D} \backslash \operatorname{Ind} \Phi$; en particulier M commute à $\operatorname{diag}(1,2)$ d'où

$$
\Phi(\mathrm{M})=\Phi(\operatorname{diag}(1,2) \mathrm{M} \operatorname{diag}(1,1 / 2)) .
$$

Puisque $\Phi$ est compatible avec la conjugaison nous avons

$$
\Phi(\mathrm{M})=\operatorname{diag}(1,2) \Phi(\mathrm{M}) \operatorname{diag}(1,1 / 2) ;
$$

cette égalité assure que $\Phi(\mathrm{M})$ appartient à $\mathcal{D}$.

Remarque 2.2. Si $\Phi$ est dominante, nous avons l'égalité $\overline{\Phi(\mathcal{D} \backslash \operatorname{Ind} \Phi)}=\mathcal{D}$ où l'adhérence est prise au sens ordinaire.

Si $\Phi$ est compatible à la conjugaison nous avons

$$
\Phi\left(\operatorname{diag}\left(\lambda_{1}, \lambda_{2}\right)\right)=\operatorname{diag}\left(\varphi_{1}\left(\lambda_{1}, \lambda_{2}\right), \varphi_{2}\left(\lambda_{1}, \lambda_{2}\right)\right)
$$

où les $\varphi_{i}$ sont rationnels (Lemme 2.1). Mais comme les matrices $\operatorname{diag}\left(\lambda_{1}, \lambda_{2}\right)$ et $\operatorname{diag}\left(\lambda_{2}, \lambda_{1}\right)$ sont conjuguées par $\left[\begin{array}{ll}0 & 1 \\ 1 & 0\end{array}\right]$ nous avons

$$
\Phi\left(\operatorname{diag}\left(\lambda_{2}, \lambda_{1}\right)\right)=\operatorname{diag}\left(\varphi_{1}\left(\lambda_{2}, \lambda_{1}\right), \varphi_{2}\left(\lambda_{2}, \lambda_{1}\right)\right)=\operatorname{diag}\left(\varphi_{2}\left(\lambda_{1}, \lambda_{2}\right), \varphi_{1}\left(\lambda_{1}, \lambda_{2}\right)\right) .
$$

Par suite

$$
\Phi\left(\operatorname{diag}\left(\lambda_{1}, \lambda_{2}\right)\right)=\operatorname{diag}\left(\varphi_{1}\left(\lambda_{1}, \lambda_{2}\right), \varphi_{1}\left(\lambda_{2}, \lambda_{1}\right)\right) .
$$

Inversement soit $\Psi: \mathbb{C}^{2} \rightarrow \mathbb{C}$ une fonction rationnelle; si $\mathrm{M}$ est une matrice $2 \times 2$ générique, $\mathrm{M}$ s'écrit $\mathrm{P} \operatorname{diag}\left(\lambda_{1}, \lambda_{2}\right) \mathrm{P}^{-1}$ et on définit

$$
\Phi(\mathrm{M})=\mathrm{P} \operatorname{diag}\left(\Psi\left(\lambda_{1}, \lambda_{2}\right), \Psi\left(\lambda_{2}, \lambda_{1}\right)\right) \mathrm{P}^{-1} .
$$

Cette définition ne dépend ni du choix de $\mathrm{P}$, ni de l'ordre choisi pour énumérer les valeurs propres. Par suite $\Phi$ s'étend en une application rationnelle de $\mathcal{M}(2 ; \mathbb{C})$ dans $\mathcal{M}(2 ; \mathbb{C})$.

Les propriétés et la dynamique de $\Phi$ sont essentiellement codées par celles de l'application correspondante $\left(\Psi\left(\lambda_{1}, \lambda_{2}\right), \Psi\left(\lambda_{2}, \lambda_{1}\right)\right)$. En particulier nous avons la :

Proposition 2.3. Soit $\Phi: \mathcal{M}(2 ; \mathbb{C}) \rightarrow \mathcal{M}(2 ; \mathbb{C})$ une application rationnelle compatible à la conjugaison; $\Phi$ est birationnelle si et seulement si sa restriction $\Psi=$ $\Phi_{\mid \mathcal{D}}: \mathcal{D} \rightarrow \mathcal{D}$ l'est.

Démonstration. Supposons que $\Psi=\Phi_{\mid \mathcal{D}}$ soit birationnelle, ou ce qui revient au même génériquement injective. Soient $\mathrm{A}, \mathrm{B}$ dans $\mathcal{M}(2 ; \mathbb{C})$ diagonalisables, i.e. $\mathrm{A}=$ $\operatorname{Pdiag}\left(\lambda_{1}, \lambda_{2}\right) \mathrm{P}^{-1}$ et $\mathrm{B}=\mathrm{Qdiag}\left(\mu_{1}, \mu_{2}\right) \mathrm{Q}^{-1}$. Si $\Phi(\mathrm{A})=\Phi(\mathrm{B})$, nous avons

$$
\mathrm{P} \operatorname{diag}\left(\Psi\left(\lambda_{1}, \lambda_{2}\right), \Psi\left(\lambda_{2}, \lambda_{1}\right)\right) \mathrm{P}^{-1}=\mathrm{Q} \operatorname{diag}\left(\Psi\left(\mu_{1}, \mu_{2}\right), \Psi\left(\mu_{2}, \mu_{1}\right)\right) \mathrm{Q}^{-1} .
$$


Par suite $\Psi\left(\lambda_{1}, \lambda_{2}\right)$ vaut $\Psi\left(\mu_{1}, \mu_{2}\right)$ ou $\Psi\left(\mu_{2}, \mu_{1}\right)$. Quitte à modifier $Q$ nous pouvons supposer que $\Psi\left(\lambda_{1}, \lambda_{2}\right)=\Psi\left(\mu_{1}, \mu_{2}\right)$ et $\Psi\left(\lambda_{2}, \lambda_{1}\right)=\Psi\left(\mu_{2}, \mu_{1}\right)$. Puisque $\Psi=\Phi_{\mid \mathcal{D}}$ est injective nous avons $\left(\lambda_{1}, \lambda_{2}\right)=\left(\mu_{1}, \mu_{2}\right)$. Ainsi

$$
\mathrm{A}=\mathrm{P} \operatorname{diag}\left(\lambda_{1}, \lambda_{2}\right) \mathrm{P}^{-1} \quad \text { et } \quad \mathrm{B}=\mathrm{Q} \operatorname{diag}\left(\mu_{1}, \mu_{2}\right) \mathrm{Q}^{-1} .
$$

L'égalité $\Phi(\mathrm{A})=\Phi(\mathrm{B})$ implique $\mathrm{Q}^{-1} \mathrm{P} \operatorname{diag}\left(\Psi\left(\lambda_{1}, \lambda_{2}\right), \Psi\left(\lambda_{2}, \lambda_{1}\right)\right) \mathrm{P}^{-1} \mathrm{Q}=$ $\operatorname{diag}\left(\Psi\left(\lambda_{1}, \lambda_{2}\right), \Psi\left(\lambda_{2}, \lambda_{1}\right)\right)$; il en résulte que $\mathrm{Q}^{-1} \mathrm{P}$ est une matrice diagonale $\mathrm{D}$. Par suite

$$
\mathrm{A}=\mathrm{P} \operatorname{diag}\left(\lambda_{1}, \lambda_{2}\right) \mathrm{P}^{-1}=\mathrm{QD} \operatorname{diag}\left(\lambda_{1}, \lambda_{2}\right) \mathrm{D}^{-1} \mathrm{Q}^{-1}=\mathrm{Q} \operatorname{diag}\left(\lambda_{1}, \lambda_{2}\right) \mathrm{Q}^{-1}=\mathrm{B} .
$$

Ainsi si $\Psi$ est birationnelle, alors $\Phi$ l'est.

Dans le même ordre d'idée on peut se demander si une transformation polynomiale (resp. un automorphisme polynomial) de $\mathcal{D}$ dans lui-même du type $\left(\Psi\left(\lambda_{1}, \lambda_{2}\right), \Psi\left(\lambda_{2}, \lambda_{1}\right)\right)$ induit une transformation polynomiale (resp. un automorphisme polynomial) équivariante de $\mathcal{M}(2 ; \mathbb{C})$ dans lui-même.

Désignons par $\tau$ l'involution de $\mathbb{C}^{2}$ définie par $\tau\left(\lambda_{1}, \lambda_{2}\right)=\left(\lambda_{2}, \lambda_{1}\right)$.

Proposition 2.4. Soit $\eta: \mathcal{D} \rightarrow \mathcal{D}$ une transformation polynomiale $\tau$-équivariante, i.e. du type

$$
\eta\left(\lambda_{1}, \lambda_{2}\right)=\left(\Psi\left(\lambda_{1}, \lambda_{2}\right), \Psi\left(\lambda_{2}, \lambda_{1}\right)\right) .
$$

Alors $\eta$ s'étend en une application $\Phi: \mathcal{M}(2 ; \mathbb{C}) \rightarrow \mathcal{M}(2 ; \mathbb{C})$ polynomiale et compatible à la conjugaison.

Démonstration. Soit $\Delta \subset \mathcal{M}(2 ; \mathbb{C})$ l'hypersurface discriminante : $\Delta=\{\mathrm{M} \in$ $\left.\mathcal{M}(2 ; \mathbb{C}) \mid(\operatorname{tr} M)^{2}-4 \operatorname{det} \mathrm{M}=0\right\}$. Sa trace sur $\mathcal{D}$ est constituée des multiples de l'identité : $\mathcal{D} \cap \Delta=\left\{\lambda \mathrm{Id} \mid \lambda \in \mathbb{C}^{*}\right\}$. Comme nous l'avons vu on peut étendre $\eta$ en une application rationnelle $\Phi: \mathcal{M}(2 ; \mathbb{C}) \rightarrow \mathcal{M}(2 ; \mathbb{C})$. Par construction $\Phi$ est holomorphe en restriction à $\mathcal{M}(2 ; \mathbb{C}) \backslash \Delta$. Soit $\mathrm{M}_{0}=\lambda_{0}$ Id un point de $\mathcal{D} \cap \Delta$; cette même construction assure que $\Phi$ reste bornée sur un petit voisinage $\mathcal{V}\left(\mathrm{M}_{0}\right)$ de $\mathrm{M}_{0}$ privé de $\Delta$. Il résulte du théorème d'HaRTOGs que $\Phi$ s'étend holomorphiquement à $\mathcal{V}\left(\mathrm{M}_{0}\right)$. Ce raisonnement montre qu'en fait $\Phi$ est holomorphe sur un voisinage de $\mathcal{D} \cap \Delta$. Mais si $\mathrm{M}$ appartient à $\Delta$, l'orbite adjointe de $\mathrm{M}$ coupe ce voisinage. Par conséquent $\Phi$ s'étend holomorphiquement à $\mathcal{M}(2 ; \mathbb{C})$. Comme $\Phi$ est rationnelle nous obtenons le résultat annoncé.

Les Propositions 2.3 et 2.4 impliquent l'énoncé suivant.

Corollaire 2.5. Un automorphisme polynomial $\tau$-équivariant de $\mathcal{D}$ dans lui-même s'étend de façon unique en un automorphisme polynomial de $\mathcal{M}(2 ; \mathbb{C})$ dans $\mathcal{M}(2 ; \mathbb{C})$ compatible à la conjugaison.

Définitions. Soient $f: X \rightarrow X$ une application rationnelle dominante et $\mathcal{F}$ un feuilletage sur $X$; on dit que $\mathcal{F}$ est invariant par $f$ si $f^{*} \mathcal{F}=\mathcal{F}$. Plus précisément si $m$ est un point générique de $X$, alors $\mathcal{F}$ est régulier en $m$, i.e. donné par les niveaux d'une submersion locale $g: \mathcal{V}(m) \rightarrow \mathbb{C}^{k}$ d'un voisinage de $m$ dans $\mathbb{C}^{k}$; de même $m$ est générique pour $f$, i.e. $m$ est une valeur régulière de $f$. Par suite $g \circ f$ est une submersion en chaque point de $f^{-1}(m)$ ce qui permet de définir les feuilles locales de $f^{*} \mathcal{F}$ comme les niveaux de $g \circ f$. Supposons que $\mathcal{F}$ soit défini par une fibration, i.e. la feuille générique de $\mathcal{F}$ est la fibre générique d'une application rationnelle $g: X \rightarrow Y$; on dit que $f$ préserve la fibration $\mathcal{F}$ si $f^{*} \mathcal{F}=\mathcal{F}$. On dit que $f$ préserve la fibration $\mathcal{F}$ fibre à fibre si $g \circ f=g$. 
Définitions. Un automorphisme élémentaire de $\mathbb{C}^{2}$ est, à conjugaison près (dans le groupe des automorphismes), de la forme suivante

$$
(\alpha x+P(y), \beta y+\gamma), \quad \alpha, \beta \in \mathbb{C}^{*}, \gamma \in \mathbb{C}, P \in \mathbb{C}[y] .
$$

Une transformation de HÉNON est par définition une transformation de la forme $(y, P(y)-\delta x)$ où $\delta$ désigne un élément de $\mathbb{C}^{*}$ et $P$ un élément de $\mathbb{C}[y]$ de degré supérieur ou égal à 2 .

Remarque 2.6. Les automorphismes élémentaires correspondent précisément aux automorphismes polynomiaux qui préservent une fibration rationnelle.

Étant donné un automorphisme polynomial $f$ de $\mathbb{C}^{2}$, nous avons l'alternative suivante $(\underline{6})$

- $f$ est conjugué à un automorphisme élémentaire;

- $f$ est conjugué à un produit de transformations de HÉNON; dans ce cas on dit que $f$ est de type HÉNON généralisé.

Soit $\eta$ un automorphisme polynomial équivariant de $\mathcal{D}$ dans lui-même, i.e. $\eta$ commute à la permutation $\tau=\left(\lambda_{2}, \lambda_{1}\right)$. En particulier sa ligne de points fixes $y=x$ est préservée par $\eta$ qui du coup possède une courbe algébrique invariante. Il s'en suit que $\eta$ ne peut être de type HÉNON généralisé (un tel automorphisme ne préserve pas de courbe algébrique, 1]). Il est nécessairement élémentaire; nous en déduisons que $\eta$ laisse invariante une fibration, d'où le résultat suivant.

Théorème 2.7. Soit $\Phi: \mathcal{M}(2 ; \mathbb{C}) \rightarrow \mathcal{M}(2 ; \mathbb{C})$ un automorphisme polynomial compatible à la conjugaison. Alors $\Phi_{\mid \mathcal{D}}$ est un automorphisme élémentaire; en particulier $\Phi$ préserve une fibration $\mathcal{L}: \mathcal{M}(2 ; \mathbb{C}) \rightarrow \mathbb{C}$ polynomiale transverse à $\mathcal{D}$ au sens où $\mathcal{L}_{\mid \mathcal{D}}: \mathcal{D} \rightarrow \mathbb{C}$ est non constante.

Exemple 2.8. L'automorphisme polynomial $\left(\lambda_{1}, \lambda_{2}\right) \mapsto\left(\lambda_{1}+\left(\lambda_{2}-\lambda_{1}\right)^{2}, \lambda_{2}+\right.$ $\left.\left(\lambda_{1}-\lambda_{2}\right)^{2}\right)$ de $\mathcal{D}$ dans $\mathcal{D}$ se relève en

$$
\left[\begin{array}{ll}
x & y \\
z & t
\end{array}\right] \mapsto\left[\begin{array}{cc}
x+(t-x)^{2}+4 y z & y \\
z & t+(t-x)^{2}+4 y z
\end{array}\right] .
$$

Ici la fibration invariante $\mathcal{L}$ est $(x-t)=$ cte. Notons que l'hypersurface discriminante $\left\{\mathrm{M} \in \mathcal{M}(2 ; \mathbb{C}) \mid(\operatorname{trM})^{2}-4 \operatorname{det} \mathrm{M}=0\right\}$ est fixée par $F$; on peut vérifier que c'est exactement l'ensemble des points fixes Fix $F$ de $F$.

Remarquons que la fibration $y / z=$ cte est invariante; nous verrons plus loin que ceci est un fait général.

La proposition qui suit se vérifie par un simple calcul formel. Elle donne en particulier une version effective des énoncés 2.32 .4 et 2.5.

Proposition 2.9. L'application rationnelle $\tau$-équivariante $\eta:\left(\lambda_{1}, \lambda_{2}\right) \rightarrow$ $\left(\Psi\left(\lambda_{1}, \lambda_{2}\right), \Psi\left(\lambda_{2}, \lambda_{1}\right)\right)$ se relève en l'application $\Phi: \mathcal{M}(2 ; \mathbb{C}) \rightarrow \mathcal{M}(2 ; \mathbb{C})$ compatible à la conjugaison définie par

$$
\left[\begin{array}{ll}
x & y \\
z & t
\end{array}\right] \mapsto\left[\begin{array}{ll}
\phi_{1} & \phi_{2} \\
\phi_{3} & \phi_{4}
\end{array}\right]
$$


où

$$
\begin{gathered}
\phi_{1}=\frac{1}{2}\left(\Psi\left(\xi_{1}, \xi_{2}\right)+\Psi\left(\xi_{2}, \xi_{1}\right)+\frac{(t-x)}{\Delta}\left(\Psi\left(\xi_{2}, \xi_{1}\right)-\Psi\left(\xi_{1}, \xi_{2}\right)\right)\right), \\
\phi_{2}=\frac{y}{\Delta}\left(\Psi\left(\xi_{1}, \xi_{2}\right)-\Psi\left(\xi_{2}, \xi_{1}\right)\right), \\
\phi_{3}=\frac{z}{\Delta}\left(\Psi\left(\xi_{1}, \xi_{2}\right)-\Psi\left(\xi_{2}, \xi_{1}\right)\right), \\
\phi_{4}=\frac{1}{2}\left(\Psi\left(\xi_{1}, \xi_{2}\right)+\Psi\left(\xi_{2}, \xi_{1}\right)+\frac{(t-x)}{\Delta}\left(\Psi\left(\xi_{1}, \xi_{2}\right)-\Psi\left(\xi_{2}, \xi_{1}\right)\right)\right), \\
\Delta=\sqrt{(t-x)^{2}+4 y z} \\
\xi_{1}=\frac{1}{2}(t+x+\Delta) \quad \text { et } \quad \xi_{2}=\frac{1}{2}(t+x-\Delta) .
\end{gathered}
$$

Dans les formules ci-dessus nous choisissons une détermination de la racine de $(t-x)^{2}+4 y z$. Un calcul de monodromie élémentaire montre que les composantes de $\Phi$ sont uniformes. Elles sont rationnelles (resp. polynomiales) si $\eta$ l'est.

\subsection{Exemples.}

Applications de la Proposition 2.9, - Les applications $\eta: \mathcal{D} \rightarrow \mathcal{D}$ linéaires

$\tau$-équivariantes sont du type $\eta\left(\lambda_{1}, \lambda_{2}\right)=\left(a \lambda_{1}+b \lambda_{2}, b \lambda_{1}+a \lambda_{2}\right)$. Une telle application se relève en

$$
\left[\begin{array}{ll}
x & y \\
z & t
\end{array}\right] \mapsto\left[\begin{array}{cc}
a x+b t & y(a-b) \\
z(a-b) & a t+b x
\end{array}\right]
$$

Ces transformations forment une algèbre commutative de dimension 2. Remarquons que $\eta$ est inversible si et seulement si $a^{2}-b^{2} \neq 0$; les inversibles constituent un groupe abélien.

- Une transformation homographique $\eta$ commute à l'involution $\tau$ si et seulement si elle est de la forme

$$
\eta\left(\lambda_{1}, \lambda_{2}\right)=\left(\frac{a \lambda_{1}+b \lambda_{2}+c}{A\left(\lambda_{1}+\lambda_{2}\right)+C}, \frac{a \lambda_{2}+b \lambda_{1}+c}{A\left(\lambda_{1}+\lambda_{2}\right)+C}\right) .
$$

Elle se relève en

$$
\left[\begin{array}{ll}
x & y \\
z & t
\end{array}\right] \mapsto\left[\begin{array}{cc}
\frac{a x+b t+c}{A(x+t)+C} & \frac{y(a-b)}{A(x+t)+C} \\
\frac{z(a-b)}{A(x+t)+C} & \frac{a t+b x+c}{A(x+t)+C}
\end{array}\right]
$$

L'ensemble de ces relevés (inversibles) forme un groupe qui est le projectivisé du groupe $\mathrm{G}$ engendré par les matrices inversibles

$$
\left[\begin{array}{ccccc}
a & b & c & 0 & 0 \\
b & a & c & 0 & 0 \\
A & A & C & 0 & 0 \\
0 & 0 & 0 & a-b & 0 \\
0 & 0 & 0 & 0 & a-b
\end{array}\right]
$$


son algèbre $\mathfrak{g}$ est engendrée par les matrices $\mathrm{X}, \mathrm{Y}, \mathrm{Z}, \mathrm{U}$ et $\mathrm{V}$ données par

$$
\begin{aligned}
\mathrm{X} & =\left[\begin{array}{ccccc}
1 & 1 & 0 & 0 & 0 \\
1 & 1 & 0 & 0 & 0 \\
0 & 0 & -2 & 0 & 0 \\
0 & 0 & 0 & 0 & 0 \\
0 & 0 & 0 & 0 & 0
\end{array}\right], \quad \mathrm{Y}=\left[\begin{array}{lllll}
0 & 0 & 1 & 0 & 0 \\
0 & 0 & 1 & 0 & 0 \\
0 & 0 & 0 & 0 & 0 \\
0 & 0 & 0 & 0 & 0 \\
0 & 0 & 0 & 0 & 0
\end{array}\right], \quad \mathrm{Z}=\left[\begin{array}{llllll}
0 & 0 & 0 & 0 & 0 \\
0 & 0 & 0 & 0 & 0 \\
1 & 1 & 0 & 0 & 0 \\
0 & 0 & 0 & 0 & 0 \\
0 & 0 & 0 & 0 & 0
\end{array}\right], \\
\mathrm{U} & =\left[\begin{array}{lllll}
1 & 0 & 0 & 0 & 0 \\
0 & 1 & 0 & 0 & 0 \\
0 & 0 & 0 & 0 & 0 \\
0 & 0 & 0 & 1 & 0 \\
0 & 0 & 0 & 0 & 1
\end{array}\right], \quad \mathrm{V}=\left[\begin{array}{ccccc}
0 & 1 & 0 & 0 & 0 \\
1 & 0 & 0 & 0 & 0 \\
0 & 0 & 0 & 0 & 0 \\
0 & 0 & 0 & -1 & 0 \\
0 & 0 & 0 & 0 & -1
\end{array}\right] .
\end{aligned}
$$

L'algèbre engendrée par X, Y et Z est isomorphe à $\mathfrak{s l}(2 ; \mathbb{C})$. On peut vérifier que $\mathfrak{g}$ est un produit semi-direct de l'algèbre commutative engendrée par $U$, $\mathrm{V}$ et de celle engendrée par $\mathrm{X}, \mathrm{Y}$ et $\mathrm{Z}$.

Le Théorème 2.7. ou le fait qu'il n'y ait pas d'automorphisme de type HÉnON généralisé commutant à $(y, x)$, ne permet pas de construire un automorphisme de $\mathcal{M}(2 ; \mathbb{C})$ compatible à la conjugaison dont la restriction à $\mathcal{D}$ soit de type HÉnON généralisé. Par contre on peut construire des transformations birationnelles compatibles dont la restriction à $\mathcal{D}$ est birationnellement conjuguée à un automorphisme de HÉnon. Dans l'exemple qui suit nous «tordons » un automorphisme de HÉnON afin d'obtenir une transformation birationnelle qui commute à $\tau$, transformation qui s'étend à $\mathcal{M}(2 ; \mathbb{C})$.

Exemple 2.10. Soit $\eta$ la transformation birationnelle définie par

$$
\begin{aligned}
\eta(x, y)= & \left(\frac{3 x^{3}-x^{2} y+5 x y^{2}+y^{3}-10 x^{2}-4 x y-10 y^{2}+12 x+12 y-8}{(x+y-2)(x-y)^{2}},\right. \\
& \left.\frac{x^{3}+5 x^{2} y-x y^{2}+3 y^{3}-10 x^{2}-4 x y-10 y^{2}+12 x+12 y-8}{(x+y-2)(x-y)^{2}}\right)
\end{aligned}
$$

notons que $\eta$ et $\tau$ commutent. Pour obtenir $\eta$ nous avons conjugué l'automorphisme de HÉNON $h=\left(y, y^{3}-x\right)$, automorphisme qui commute à $(-x,-y)$, par la transformation $\left(\frac{x+y+1}{x}, \frac{x+y-1}{x}\right)$ qui conjugue $(-x,-y)$ à $\tau$. Ce $\eta$ se relève en l'application $\Phi$ de $\mathcal{M}(2 ; \mathbb{C})$ dans lui-même donnée par

$$
\left[\begin{array}{ll}
x & y \\
z & t
\end{array}\right] \mapsto\left[\begin{array}{ll}
\phi_{1} & \phi_{2} \\
\phi_{3} & \phi_{4}
\end{array}\right]
$$

où

$$
\begin{gathered}
\phi_{1}=\frac{3 x^{3}+t^{3}+5 x t^{2}+8 x y z-x^{2} t-4 x t-10\left(t^{2}+x^{2}\right)-16 y z+12(t+x)-8}{\left((t-x)^{2}+4 y z\right)(t+x-2)}, \\
\phi_{2}=\frac{2 y}{t+x-2}, \quad \phi_{3}=\frac{2 z}{t+x-2}, \\
\phi_{4}=\frac{x^{3}+3 t^{3}-x t^{2}+5 x^{2} t+8 y z t-10\left(x^{2}+t^{2}\right)-4 x t-16 y z+12(t+x)-8}{\left((t-x)^{2}+4 y z\right)(t+x-2)} .
\end{gathered}
$$

La transformation birationnelle $\Phi$ hérite des propriétés de l'automorphisme de HÉnON : en particulier l'ensemble de Julia de $h$ induit un ensemble de « type JuLIA $\gg$ invariant par $\Phi$ et l'action adjointe de $\operatorname{GL}(2 ; \mathbb{C}) \operatorname{sur} \mathcal{M}(2 ; \mathbb{C})$. 
2.3. Fibrations, feuilletages, tissus, coniques invariants et application squelette. Revenons encore à $\Phi_{\mathrm{Id}}(\mathrm{M})=\mathrm{M}^{2}$ pour laquelle nous allons dégager un ensemble de fibrations invariantes pour certaines « universelles $\gg$. Comme on l'a vu, le pinceau d'hyperplans associé à la fibration $y / z$ est invariant par $\Phi_{\text {Id }}$ fibre à fibre, ce fait se généralise à toute application compatible à la conjugaison (Proposition 2.9) :

Proposition 2.11. Soit $\Phi: \mathcal{M}(2 ; \mathbb{C}) \rightarrow \mathcal{M}(2 ; \mathbb{C})$ une application rationnelle compatible à la conjugaison. La fibration donnée par les niveaux de $y / z$ et le feuilletage associé au champ y $\frac{\partial}{\partial y}-z \frac{\partial}{\partial z}$ sont invariants par $\Phi$.

Remarque 2.12. Sous les hypothèses de la proposition précédente le pinceau d'hyperplans associé à la fibration $y / z$ est invariant par $\Phi$ fibre à fibre, i.e. les orbites de $\Phi$ sont contenues dans les hyperplans $y / z=$ cte.

Le flot du champ de vecteurs $y \frac{\partial}{\partial y}-z \frac{\partial}{\partial z}$ est donné par $\left(x, y \mathrm{e}^{s}, z \mathrm{e}^{-s}, t\right)$. Ses trajectoires sont les fibres de la fibration en coniques $\mathcal{M}(2 ; \mathbb{C}) \rightarrow \mathbb{C}^{3},(x, y, z, t) \mapsto$ $(x, y z, t)$. La fibration précédente est conservée globalement par $\Phi$ mais pas fibre à fibre.

Notons que $\Phi_{\text {Id }}$ laisse aussi la fibration $\frac{x-t}{y}=$ cte invariante fibre à fibre.

Considérons dans $\mathcal{M}(2 ; \mathbb{C})$ la droite $\mathcal{C}=\{\lambda \mathrm{Id} \mid \lambda \in \mathbb{C}\}$, i.e. le centre de $\mathcal{M}(2 ; \mathbb{C})$ pour sa loi d'algèbre. À $\mathcal{C}$ on peut associer la fibration $\mathcal{P}$ en 2-plans définie comme suit : si $\mathrm{M}$ appartient à $\mathcal{M}(2 ; \mathbb{C}) \backslash \mathcal{C}$, on définit $\mathcal{P}(\mathrm{M})$ comme l'unique plan contenant $\mathrm{M}$ et $\mathcal{C}$. Si $\mathrm{M}$ est un élément de $\mathcal{M}(2 ; \mathbb{C})$, alors $\mathcal{P}(\mathrm{M})$ est exactement l'ensemble des matrices qui commutent à $\mathrm{M}$. On peut aussi remarquer que le plan générique $\mathcal{P}(\mathrm{M})$ est un conjugué de $\mathcal{D}$ : si $\mathrm{P}$ diagonalise $\mathrm{M}$, i.e. $\mathrm{PMP}^{-1}$ appartient à $\mathcal{D}$, alors $\mathcal{P}(\mathrm{M})$ est inclu dans $\mathrm{PDP}^{-1}$ et donc égal. Ceci induit un feuilletage singulier en 2-plans qui est invariant par $\Phi_{\text {Id }}$ feuille à feuille. En effet soit $s \mathrm{M}+t \mathrm{Id}$ dans $\mathcal{P}(\mathrm{M})$; on constate que

$$
\Phi_{\text {Id }}(s \mathrm{M}+t \mathrm{Id})=s^{2} \mathrm{M}^{2}+2 s t \mathrm{M}+t^{2} \mathrm{Id}
$$

commute visiblement à M. Par suite les orbites de $\Phi_{\text {Id }}$ sont dans les 2-plans du pinceau $\mathcal{P}$. Ainsi l'application $\mathbb{P}\left(\Phi_{\mathrm{Id}}\right): \mathbb{P}^{3}(\mathbb{C}) \rightarrow \mathbb{P}^{3}(\mathbb{C})$ laisse invariante la fibration en droites $\mathbb{P}(\mathcal{P})$ : ce sont les droites de $\mathbb{P}^{3}(\mathbb{C})$ passant par $[\mathrm{Id}]=(1: 0: 0$ : $1)$. Nous avons des propriétés analogues pour les transformations $\Phi_{\alpha, d}$ homogènes compatibles à la conjugaison.

Si M est un point générique de $\mathcal{M}(2 ; \mathbb{C})$ l'adhérence de ZARISKI de son orbite par $\Phi_{\text {Id }}$ est un 2-plan (car c'est le cas pour un élément générique de $\mathcal{D}$ ). Par conséquent il n'y a qu'une seule fibration en surfaces invariante fibre à fibre par $\Phi_{\mathrm{Id}}$, la fibration $\mathcal{P}$. Notamment la fibration $\mathcal{P}$ est donnée par les niveaux de l'application rationnelle $\left(\frac{y}{z}, \frac{x-t}{y}\right)$ ce que l'on peut voir de façon directe.

En fait l'invariance de $\mathcal{P}$ se généralise à toutes les applications compatibles à la conjugaison.

Proposition 2.13. Soit $\Phi: \mathcal{M}(2 ; \mathbb{C}) \rightarrow \mathcal{M}(2 ; \mathbb{C})$ une application rationnelle compatible à la conjugaison; $\Phi$ préserve la fibration en 2-plans $\mathcal{P}$ fibre à fibre.

Démonstration. D'après le Lemme 2.1 nous avons $\Phi(\mathcal{D} \backslash$ Ind $\Phi) \subset \mathcal{D}$. Comme un plan générique $\mathcal{P}(\mathrm{M})$ de $\mathcal{P}$ est un conjugué de $\mathcal{D}, \mathcal{P}(\mathrm{M})$ est invariant par $\Phi$, d'où le résultat. 
Remarque 2.14. Comme on l'a vu, par définition de la fibration $\mathcal{P}, \Phi$ préserve $\mathcal{P}$ fibre à fibre si et seulement si $\Phi(\mathrm{M})$ commute à $\mathrm{M}$ pour tout $\mathrm{M}$ dans $\mathcal{M}(2 ; \mathbb{C})$. Ceci permet de vérifier qu'il existe des applications préservant $\mathcal{P}$ qui ne sont pas compatibles à la conjugaison.

La proposition qui suit est conséquence directe de la définition de la compatibilité.

Proposition 2.15. Soit $\Phi: \mathcal{M}(2 ; \mathbb{C}) \rightarrow \mathcal{M}(2 ; \mathbb{C})$ une application rationnelle compatible à la conjugaison. Alors $\Phi$ laisse invariant le feuilletage associé à la fibration Inv. Plus précisément il existe une application rationnelle $\mathrm{Sq} \Phi: \mathbb{C}^{2} \rightarrow \mathbb{C}^{2}$ telle que l'on ait le diagramme commutatif suivant

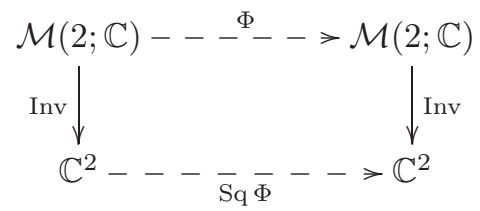

Remarque 2.16. Les fibres de Inv sont des surfaces quadratiques : $x+t=$ cte, $x t-y z=$ cte.

Remarque 2.17. Il y a des applications qui préservent la fibration Inv sans être compatible à la conjugaison : par exemple soit $\mathrm{M} \mapsto P(\mathrm{M})$ une application polynomiale de $\mathcal{M}(2 ; \mathbb{C})$ dans lui-même telle que $\operatorname{det} P \not \equiv 0$. Alors l'application $\mathrm{M} \mapsto$ $(P(\mathrm{M}))^{-1} \mathrm{M} P(\mathrm{M})$ respecte la fibration Inv mais n'est pas, en général, compatible à la conjugaison.

Inversement on peut se demander à quelle condition une application rationnelle de $\mathbb{C}^{2}$ se relève à $\mathcal{M}(2 ; \mathbb{C})$ via Inv. La réponse est donnée par l'énoncé suivant :

Proposition 2.18. Soit $W: \mathbb{C}^{2} \rightarrow \mathbb{C}^{2},(u, v) \rightarrow(S(u, v), T(u, v))$ une application rationnelle. Alors $W$ s'écrit $\mathrm{Sq} \Phi$ pour une certaine application rationnelle $\Phi: \mathcal{M}(2 ; \mathbb{C}) \rightarrow \mathcal{M}(2 ; \mathbb{C})$ compatible à la conjugaison si et seulement si $\left(S^{2}-4 T\right) \circ$ Inv est un carré dans le corps des fonctions rationnelles $\mathbb{C}(u, v)$.

Démonstration. Par restriction aux matrices diagonales il suffit de résoudre en $\Psi$ :

$$
\begin{gathered}
S\left(\lambda_{1}+\lambda_{2}, \lambda_{1} \lambda_{2}\right)=\Psi\left(\lambda_{1}, \lambda_{2}\right)+\Psi\left(\lambda_{2}, \lambda_{1}\right), \\
T\left(\lambda_{1}+\lambda_{2}, \lambda_{1} \lambda_{2}\right)=\Psi\left(\lambda_{1}, \lambda_{2}\right) \Psi\left(\lambda_{2}, \lambda_{1}\right) .
\end{gathered}
$$

Par élimination de $\Psi\left(\lambda_{2}, \lambda_{1}\right)$ nous obtenons

$$
\Psi^{2}\left(\lambda_{1}, \lambda_{2}\right)-S\left(\lambda_{1}+\lambda_{2}, \lambda_{1} \lambda_{2}\right) \Psi\left(\lambda_{1}, \lambda_{2}\right)+T\left(\lambda_{1}+\lambda_{2}, \lambda_{1} \lambda_{2}\right)=0
$$

d'où le résultat.

Exemple 2.19. L'application $\operatorname{Sq} \Phi_{\text {Id }}$ est donnée par $\operatorname{Sq} \Phi_{\text {Id }}(u, v)=\left(u^{2}-2 v, v^{2}\right)$. Alors que l'extension $\widetilde{\Phi_{\text {Id }}}$ de $\Phi_{\text {Id }}$ à $\mathbb{P}^{4}(\mathbb{C})$ n'est pas un endomorphisme (puisque la quadrique $\{\mathrm{M} \in \mathcal{M}(2 ; \mathbb{C}) \mid \operatorname{Inv}(\mathrm{M})=(0,0)\}$ est d'indétermination), celle de $\mathrm{Sq} \Phi_{\mathrm{Id}}$ à $\mathbb{P}^{2}(\mathbb{C})$ l'est.

Notons que la conique $v=\frac{u^{2}}{4}$ est invariante par Sq $\Phi_{\text {Id }}$ : c'est l'image par Inv de l'hypersurface discriminante

$$
\left\{\mathrm{M} \in \mathcal{M}(2 ; \mathbb{C}) \mid 4 \operatorname{det} \mathrm{M}-(\operatorname{tr} \mathrm{M})^{2}=0\right\}
$$


c'est donc aussi l'image de l'orbite sous l'action adjointe du 2-plan :

$$
\left\{\lambda \operatorname{Id}+\mu\left[\begin{array}{ll}
0 & 1 \\
0 & 0
\end{array}\right] \mid \lambda, \mu \in \mathbb{C}\right\}=\mathcal{P}\left(\left[\begin{array}{ll}
0 & 1 \\
0 & 0
\end{array}\right]\right) .
$$

Ses tangentes définissent un «2-tissu $\gg$ complètement invariant par Sq $\Phi_{\text {Id }}$ (i.e. invariant par images directe et réciproque) :

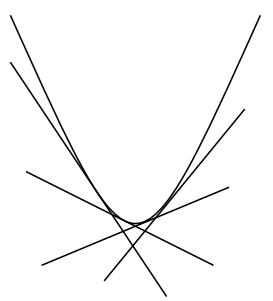

Exemple 2.20. Considérons cette fois l'application $\Phi$ compatible à la conjugaison définie par $\Phi(\mathrm{M})=\mathrm{M}+\mathrm{M}^{-1}$. Son extension $\mathbb{P}(\Phi): \mathbb{P}^{4}(\mathbb{C}) \rightarrow \mathbb{P}^{4}(\mathbb{C})$ est la transformation de degré trois

$$
\begin{array}{r}
\mathbb{P}(\Phi):(x: y: z: t: w) \mapsto\left(x \delta+t w^{2}: y\left(\delta-w^{2}\right): z\left(\delta-w^{2}\right): t \delta+x w^{2}: w \delta\right), \\
\delta=x t-y z ;
\end{array}
$$

elle préserve la fibration $y / z=$ cte. Il y a un seul point d'indétermination à distance finie; à l'infini l'ensemble d'indétermination est donné par $\delta=w=0$. Une matrice de déterminant nul va à l'infini sur sa matrice de cofacteurs.

La restriction de $\Phi$ à $\mathcal{D}$ induit l'application $\left(\lambda_{1}, \lambda_{2}\right) \mapsto\left(\lambda_{1}+\lambda_{1}^{-1}, \lambda_{2}+\lambda_{2}^{-1}\right)$. Nous en déduisons l'application Sq $\Phi: \mathbb{C}^{2} \rightarrow \mathbb{C}^{2}$ donnée par

$$
(u, v) \mapsto\left(\frac{u}{v}+u, v+\frac{u^{2}}{v}-2+\frac{1}{v}\right) .
$$

Elle se prolonge en l'endomorphisme $(u, v, w) \mapsto\left(u(v+w):(v-w)^{2}+u^{2}: v w\right)$ de $\mathbb{P}^{2}(\mathbb{C})$ qui préserve uniquement deux droites (celle d'équation $u=0$, resp. $w=$ $0)$. Ceci donne un autre exemple qui montre que l'application Sq peut avoir une extension holomorphe à $\mathbb{P}^{2}(\mathbb{C})$ alors que l'extension $\widetilde{\Phi}$ de $\Phi$ à $\mathbb{P}^{4}(\mathbb{C})$ est seulement rationnelle. La courbe $\mathcal{C}$ d'équation $v=\frac{u^{2}}{4}$ est encore invariante par $\mathrm{Sq} \Phi$. Comme dans l'Exemple 2.19 la famille des tangentes à $\mathcal{C}$ est un 2-tissu invariant par Sq $\Phi$. La famille des fonctions rationnelles $\lambda\left(z+\frac{1}{z}\right)$ sur $\mathbb{P}^{1}(\mathbb{C})$ a été étudiée en détail dans [9. Pour deux valeurs de $\lambda$ spéciales $\left(\lambda= \pm \frac{\mathrm{i}}{2}\right)$ il s'agit d'un exemple de LATTÈs. L'application induite correspondante $\zeta_{\lambda}: \mathcal{M}(2 ; \mathbb{C}) \rightarrow \mathcal{M}(2 ; \mathbb{C})$ définie par $\zeta_{\lambda}(\mathrm{M})=\lambda\left(\mathrm{M}+\mathrm{M}^{-1}\right)$ hérite de ses propriétés; par exemple pour $\lambda= \pm \frac{\mathrm{i}}{2}$ l'ensemble $\operatorname{Per} \zeta_{\lambda}$ des points périodiques de $\zeta_{\lambda}$ est dense dans $\mathcal{M}(2 ; \mathbb{C})$.

Exemple 2.21. Soit $\varsigma_{c}: \mathcal{M}(2 ; \mathbb{C}) \rightarrow \mathcal{M}(2 ; \mathbb{C})$ définie par $\varsigma_{c}(\mathrm{M})=\mathrm{M}^{2}+c \mathrm{Id}$. La restriction de $\varsigma_{c}$ à $\mathcal{D}$ induit l'application $\left(\lambda_{1}, \lambda_{2}\right) \mapsto\left(\lambda_{1}^{2}+c, \lambda_{2}^{2}+c\right)$ d'où l'application rationnelle

$$
\mathrm{Sq} \varsigma_{c}: \mathbb{C}^{2}-\rightarrow \mathbb{C}^{2}, \quad(u, v) \mapsto\left(u^{2}-2 v+2 c, v^{2}+c\left(u^{2}-2 v\right)+c^{2}\right) ;
$$

elle se prolonge en un endomorphisme de $\mathbb{P}^{2}(\mathbb{C})$. La conique $\mathcal{C}$ d'équation $v=\frac{u^{2}}{4}$ est invariante par $\mathrm{Sq} \varsigma_{c}$ et la dynamique de $\mathrm{Sq} \varsigma_{c \mid \mathcal{C}}$ est conjuguée à $z \mapsto \frac{z^{2}}{2}+2 c$ qui est conjuguée à $z \mapsto z^{2}+c\left(\right.$ via $\left.z \mapsto \frac{z}{2}\right)$. 
De plus, comme toute application compatible, $\varsigma_{c}$ laisse $y / z=$ cte et $\mathcal{P}$ invariantes fibre à fibre.

Ici encore l'hyperplan $\mathfrak{s l}(2 ; \mathbb{C})$ est contracté sur la droite $\mathbb{C} \cdot I d ;$ plus précisément $\varsigma_{c}(x, y, z,-x)=\left(x^{2}+y z\right)$ Id et la restriction de $\varsigma_{c}$ à $\mathbb{C} \cdot$ Id se traite évidemment comme $z \mapsto z^{2}+c$.

$\mathrm{Au}$ vu des exemples précédents on peut se demander si le fait que la courbe $\mathcal{C}$ d'équation $v=\frac{u^{2}}{4}$ soit invariante par $\mathrm{Sq} \Phi$ est un fait général. La proposition qui suit donne une réponse partielle.

Proposition 2.22. Soit $\Phi: \mathcal{M}(2 ; \mathbb{C}) \rightarrow \mathcal{M}(2 ; \mathbb{C})$ un automorphisme polynomial compatible à la conjugaison; l'hypersurface discriminante $\Delta=\{\mathrm{M} \in \mathcal{M}(2 ; \mathbb{C}) \mid 4 \operatorname{det} \mathrm{M}$ $\left.-(\operatorname{tr} \mathrm{M})^{2}=0\right\}$ est invariante par $\Phi$ d'où l'invariance de la conique $v=\frac{u^{2}}{4}$ par $\mathrm{Sq} \Phi$.

Démonstration. Raisonnons par l'absurde : supposons que l'hypersurface $\Delta$ ne soit pas invariante. Il existe donc $\mathrm{M}_{0}$ non diagonalisable dans $\Delta$ tel que $\Phi\left(\mathrm{M}_{0}\right)$ soit diagonalisable d'où l'existence de $\mathrm{M}_{0}^{\prime}$ dans $\Delta$ tel que $\Phi\left(\mathrm{M}_{0}^{\prime}\right)$ soit diagonale. L'ensemble $\mathcal{D} \mathrm{M}_{0}^{\prime} \mathcal{D}^{-1}$ est de dimension supérieure ou égale à 1 et, pour tout $\mathrm{D}$ dans $\mathcal{D}$, nous avons $\Phi\left(\mathrm{DM}_{0}^{\prime} \mathrm{D}^{-1}\right)=\mathrm{D} \Phi\left(\mathrm{M}_{0}^{\prime}\right) \mathrm{D}^{-1}=\Phi\left(\mathrm{M}_{0}^{\prime}\right)$ : contradiction.

Remarques 2.23. Cette démonstration s'étend aux applications polynomiales $\Phi$ de $\mathcal{M}(2 ; \mathbb{C})$ dans lui-même qui ne contractent pas de courbe sur un point. Remarquons aussi qu'une application de la forme $\frac{P(\mathrm{M})}{4 \operatorname{det} \mathrm{M}-(\operatorname{tr} \mathrm{M})^{2}}$, où $P$ désigne une application polynomiale compatible à la conjugaison, n'est pas bien définie sur $\Delta$.

L'Exemple 2.20 montre que cette conique peut aussi être invariante dans le cas non injectif.

Proposition 2.24. Soit $\Phi: \mathcal{M}(2 ; \mathbb{C}) \rightarrow \mathcal{M}(2 ; \mathbb{C})$ une transformation compatible à la conjugaison. Alors $\Phi$ est birationnelle si et seulement si $\mathrm{Sq} \Phi$ l'est.

Démonstration. Supposons Sq $\Phi$ non (génériquement) injective. Ceci signifie que $\Phi$ envoie (au moins) deux orbites de l'action adjointe sur une seule et ceci génériquement; en particulier $\Phi$ est non injective.

Réciproquement on sait que $\Phi$ est birationnelle si et seulement si $\Phi_{\mid \mathcal{D}}$ l'est. Si on identifie $\Phi_{\mid \mathcal{D}}$ à une application rationnelle $\varphi:(x, t) \mapsto(a(x, t), b(x, t))$ de $\mathbb{C}^{2}$ dans lui-même, l'injectivité de Sq $\Phi$ se traduit par celle de $(x, t) \mapsto(a(x, t)+$ $b(x, t), a(x, t) b(x, t))$ et donc celle de $\varphi$; la transformation $\Phi$ est donc birationnelle.

Remarque 2.25. Soit $\Phi: \mathcal{M}(2 ; \mathbb{C}) \rightarrow \mathcal{M}(2 ; \mathbb{C})$ une transformation compatible à la conjugaison. Si l'application $\mathrm{Sq} \Phi$ est triviale, alors $\Phi$ est triviale ou $\Phi: \mathrm{M} \mapsto$ $(\operatorname{det} \mathrm{M}) \mathrm{M}^{-1}$. En effet si Sq $\Phi=\mathrm{id}$, alors $\Phi_{\mid \mathcal{D}}$ coïncide avec $(x, t) \mapsto(x, t)$ ou $(x, t) \mapsto$ $(t, x)$; la Proposition 2.9 permet de conclure.

\section{Dynamique De $\Phi_{\text {Id }}$}

Soit $\Phi: \mathcal{M}(2 ; \mathbb{C}) \rightarrow \mathcal{M}(2 ; \mathbb{C})$ une application rationnelle; notons $K(\Phi)$ le corps des fonctions rationnelles invariantes par $\Phi$. Un élément de $K(\Phi)$ est une fonction rationnelle $f: \mathcal{M}(2 ; \mathbb{C}) \rightarrow \mathbb{P}^{1}(\mathbb{C})$ telle que $f \circ \Phi=f$.

Revenons à l'application $\Phi_{\mathrm{Id}}: \mathcal{M}(2 ; \mathbb{C}) \rightarrow \mathcal{M}(2 ; \mathbb{C}), \mathrm{M} \mapsto \mathrm{M}^{2}:$

$$
\left[\begin{array}{ll}
x & y \\
z & t
\end{array}\right] \rightarrow\left[\begin{array}{cc}
x^{2}+y z & y(x+t) \\
z(x+t) & t^{2}+y z
\end{array}\right]
$$


Comme $\Phi_{\text {Id }}$ laisse le feuilletage en 2-plans $\mathcal{P}$ invariant, chacune de ses orbites est contenue dans un certain 2-plan de $\mathcal{P}$. Au niveau algébrique ceci se formalise de la façon suivante :

Théorème 3.1. Les fonctions invariantes par $\Phi_{\mathrm{Id}}$ sont engendrées par $\frac{y}{z}$ et $\frac{x-t}{z}$, i.e. $\mathrm{K}\left(\Phi_{\mathrm{Id}}\right)=\mathbb{C}\left(\frac{y}{z}, \frac{x-t}{z}\right)$.

Démonstration. Soit $f$ une fonction rationnelle invariante par $\Phi_{\text {Id }}$. Il suffit de montrer que $f$ est constante sur chaque 2-plan de $\mathcal{P}$. Plus précisément il suffit de l'établir pour un ensemble dense de tels 2-plans, par exemple sur les 2-plans du type suivant

$$
\Pi(\mathrm{P})=\left\{\mathrm{P} \operatorname{diag}(x, t) \mathrm{P}^{-1} \mid(x, t) \in \mathbb{C}^{2}\right\}
$$

où $\mathrm{P}$ est une matrice inversible fixée. La condition d'invariance implique les égalités suivantes

$$
f\left(\mathrm{P} \operatorname{diag}\left(x^{2^{n}}, t^{2^{n}}\right) \mathrm{P}^{-1}\right)=f\left(\mathrm{P} \operatorname{diag}(x, t) \mathrm{P}^{-1}\right), \quad \text { pour tout } n \text { dans } \mathbb{Z} .
$$

Ainsi $f$ est constante sur l'orbite $\mathcal{O}\left(\mathrm{P} \operatorname{diag}\left(x_{0}, t_{0}\right) \mathrm{P}^{-1} ; \Phi_{\text {Id }}\right)$ de $\mathrm{P} \operatorname{diag}\left(x_{0}, t_{0}\right) \mathrm{P}^{-1}$ par $\Phi_{\text {Id }}$ qui, pour un choix générique de $\left(x_{0}, t_{0}\right)$, est ZARISKI dense dans $\Pi(\mathrm{P})$. Par suite $f$ est constante sur chaque $\Pi(\mathrm{P})$ et donc sur chaque 2-plan de $\mathcal{P}$.

Si $\Phi: \mathcal{M}(2 ; \mathbb{C}) \rightarrow \mathcal{M}(2 ; \mathbb{C})$ est compatible à la conjugaison, sa dynamique hérite de cette compatibilité. Par exemple si $\mathrm{M}$ est périodique pour $\Phi$, i.e. $\Phi^{k}(\mathrm{M})=\mathrm{M}$ pour un certain $k$, alors toute l'orbite

$$
\left\{\mathrm{AMA}^{-1} \mid \mathrm{A} \in \mathrm{GL}(2 ; \mathbb{C})\right\}
$$

de $\mathrm{M}$ est bien sûr périodique. De même tout ensemble $S$ invariant par $\Phi$ a son saturé $\left\{\mathrm{A} S \mathrm{~A}^{-1} \mid \mathrm{A} \in \mathrm{GL}(2 ; \mathbb{C})\right\}$ invariant par $\Phi$.

3.1. Points fixes, points périodiques. Nous nous intéressons aux points périodiques de l'application $\Phi_{\mathrm{Id}}$. Comme $\Phi_{\mathrm{Id}}$ est compatible avec la conjugaison il suffit de tester les matrices de JoRDAN du type $\left[\begin{array}{cc}\lambda_{1} & 0 \\ 0 & \lambda_{2}\end{array}\right]$ (c'est-à-dire étudier les points périodiques de $\left.\Phi_{\operatorname{Id}_{\mid \mathcal{D}}}\right)$ et $\left[\begin{array}{ll}\lambda & 1 \\ 0 & \lambda\end{array}\right]$. On vérifie que les points fixes de $\Phi_{\operatorname{Id}}$ sont $\mathbf{0}$, Id et les matrices conjuguées à $\left[\begin{array}{ll}1 & 0 \\ 0 & 0\end{array}\right]$; autrement dit les points fixes sont

$$
\{\mathbf{0}, \operatorname{Id}\} \cup\{\mathrm{M} \in \mathcal{M}(2 ; \mathbb{C}) \mid \operatorname{Inv}(\mathrm{M})=(1,0)\} .
$$

L'étude de $\Phi_{\operatorname{Id}_{\mid \mathcal{D}}}$ repose sur celle de $\chi_{2}: z \mapsto z^{2}$ dans le plan complexe. Rappelons que l'ensemble de Julia de $\chi_{2}$ est le cercle unité et que le point 0 est superattractant. Si $0<|z|<1$ les itérés de $z$ convergent vers 0 ; si $|z|>1$ les itérés de $z$ tendent vers l'infini (si on compactifie $\mathbb{C}$, l'infini est aussi super attracteur). Les points périodiques autres que 0 et $\infty$ sont donc sur le cercle unité. Pour décrire l'orbite de $\mathrm{e}^{2 \mathrm{i} \pi \vartheta}$ sous l'action de $\chi_{2}$ on choisit d'écrire $\vartheta$ sous forme 2-adique : $\vartheta=\sum_{n>1} \frac{\varepsilon_{n}}{2^{n}}$ avec $\varepsilon_{n} \in\{0,1\}$. On peut ainsi coder un point de $\mathbb{S}^{1}$ par la suite $\varepsilon=\left(\varepsilon_{1}, \varepsilon_{2}, \ldots\right)$. L'action de $\chi_{2}$ dans l'écriture 2-adique des angles est le shift $\left(\varepsilon_{1}, \varepsilon_{2}, \varepsilon_{3}, \ldots\right) \rightarrow\left(\varepsilon_{2}, \varepsilon_{3}, \varepsilon_{4}, \ldots\right)$. Un point de $\mathbb{S}^{1}$ associé à une suite $\varepsilon$ périodique est lui-même périodique pour $\chi_{2}$; tous les points périodiques sont de cette forme. Ce sont aussi les racines $\left(2^{n}-1\right)$-ième de l'unité qui sont bien sûr denses dans le cercle $\mathbb{S}^{1}$. Si $z$ est un point générique de $\mathbb{S}^{1}$, alors l'orbite de $z$ par $\chi_{2}$ est dense dans le cercle. 
En identifiant $\mathcal{D}$ à $\mathbb{C}^{2}$ et $\Phi_{\operatorname{Id}_{\mid \mathcal{D}}}$ à $\left(x^{2}, t^{2}\right)$ les points périodiques de $\Phi_{\operatorname{Id}_{\mid \mathcal{D}}}$ sont, outre $(0,0)$, de l'un des types suivants

$$
\left(x_{k_{n}}, t_{k_{n}}\right), \quad\left(x_{k_{n}}, 0\right), \quad\left(0, t_{k_{n}}\right)
$$

où les $x_{k_{n}}$ et $t_{k_{n}}$ parcourent les racines $\left(2^{n}-1\right)$-ième de l'unité. Notons que l'ensemble

$$
\left\{(x, t) \in \mathbb{S}^{1} \times \mathbb{S}^{1} \mid x^{2^{n}-1}=t^{2^{n}-1}=1, n \in \mathbb{N}\right\}
$$

est dense dans le tore $\mathbb{S}^{1} \times \mathbb{S}^{1}$. Les ensembles $\mathbb{S}^{1} \times\{0\},\{0\} \times \mathbb{S}^{1}$ et $\mathbb{S}^{1} \times \mathbb{S}^{1}$ sont $\Phi_{\mathrm{Id}_{\mid \mathcal{D}}}$-invariants, de même que les ensembles $\{|x| \leq 1,|t| \leq 1\},\{|x| \leq 1,|t|=1\}$, $\{|x| \leq 1,|t| \geq 1\}$ etc. Si $M$ est une matrice diagonalisable, alors l'orbite $\mathcal{O}\left(\mathrm{M} ; \Phi_{\text {Id }}\right)$ de $\mathrm{M}$ coïncide avec celle $\operatorname{de} \operatorname{diag}\left(\lambda_{1}, \lambda_{2}\right)$ où les $\lambda_{i}$ sont les valeurs propres de $\mathrm{M}$ de sorte que la description de l'orbite de $\mathrm{M}$ ne dépend que de ses valeurs propres. En particulier tous les éléments conjugués aux $\operatorname{diag}\left(x_{k_{n}}, t_{k_{n}}\right)$ sont périodiques de période $2^{n}-1$. Dans le même ordre d'idée si les valeurs propres $\lambda_{1}, \lambda_{2}$ de $\mathrm{M}$ sont en module strictement inférieures à 1 , alors $\lim _{k \rightarrow+\infty} \Phi_{\mathrm{Id}}^{k}(\mathrm{M})=0$.

Reste à décrire l'orbite des matrices de type $\left[\begin{array}{cc}\lambda & \lambda \\ 0 & \lambda\end{array}\right]=\lambda\left[\begin{array}{ll}1 & 1 \\ 0 & 1\end{array}\right]$ dont l'itération nième par $\Phi_{\text {Id }}$ donne $\mathrm{M}_{n}(\lambda)=\lambda^{2^{n}}\left[\begin{array}{cc}1 & 2^{n} \\ 0 & 1\end{array}\right]$.

Aucune valeur de $\lambda$ non triviale ne produit ici de point périodique :

- si $|\lambda|<1$, alors $\mathrm{M}_{n}(\lambda) \rightarrow \mathbf{0}$,

- si $|\lambda| \geq 1$, alors $\mathrm{M}_{n}(\lambda) \ll$ s'échappe $\gg$ dans un sens que nous précisons maintenant. Si on compactifie $\mathcal{M}(2 ; \mathbb{C}) \simeq \mathbb{C}^{4}$ par $\mathbb{P}^{4}(\mathbb{C})=\{(x: y: z: t: w)\}$, alors $\mathrm{M}_{n}(\lambda)$ correspond à

$$
\left(\lambda^{2^{n}}: \lambda^{2^{n}} 2^{n}: 0: \lambda^{2^{n}}: 1\right)=\left(\frac{1}{2^{n}}: 1: 0: \frac{1}{2^{n}}: \frac{1}{\lambda^{2^{n}} 2^{n}}\right)
$$

qui pour $|\lambda| \geq 1$ tend vers (0:1:0:0:0), point d'indétermination de l'extension de $\Phi_{\text {Id }}$ à $\mathbb{P}^{4}(\mathbb{C})$.

Nous déduisons de ce qui précède que $\mathbf{0}$ est un point super attractant. En effet si $\mathrm{M}$ est proche de $\mathbf{0}$, alors M est conjuguée à une matrice de JoRDAN $\left[\begin{array}{cc}\lambda_{1} & 0 \\ 0 & \lambda_{2}\end{array}\right]$ avec $\left|\lambda_{i}\right|<1$ ou à $\left[\begin{array}{ll}\lambda & \lambda \\ 0 & \lambda\end{array}\right]$ avec $|\lambda|<1$. En particulier les matrices nilpotentes sont « pré-super attractives ». Le bassin d'attraction $\mathrm{W}_{\mathrm{Id}}^{s}(\mathbf{0})$ de $\mathbf{0}$ est constitué des matrices ayant leurs deux valeurs propres de module strictement plus petit que 1. Son bord est l'ensemble des matrices $M$ ayant une valeur propre de module 1 et l'autre de module plus petit ou égal à 1. C'est une hypersurface LEvi-plate1 1 que nous allons décrire. Nous utiliserons la proposition suivante dont la preuve est élémentaire.

Proposition 3.2. Soit $P(z)=z^{2}-b z+c$ un trinôme $d u$ second degré, $(b, c)$ désignant un élément de $\mathbb{C}^{2}$. Alors $P$ a une racine de module 1 et l'autre de module

1. Rappelons qu'une sous-variété réelle $V$ de codimension 1 dans $\mathbb{C}^{n}=\mathbb{R}^{2 n}$ est dite Levi-plate si son champ d'hyperplans tangents complexes $\mathrm{T}_{m}^{\mathbb{C}} V=\mathrm{T}_{m} V \cap \mathrm{iT}_{m} V$ est intégrable. Ce champ induit alors un feuilletage de $V$ en sous-variétés complexes de dimension $n-1$. 
plus petit que 1 si et seulement si on a

$$
\begin{cases}|c|^{2}-\frac{1}{2}|b|^{2}-\frac{1}{2}\left|b^{2}-4 c\right|+1=0 & (i) \\ |c| \leq 1 & (i i)\end{cases}
$$

Démonstration. Désignons par $\lambda_{1}$ et $\lambda_{2}$ les deux racines de $P$. La première condition se traduit par

$$
\left(\left|\lambda_{1}\right|^{2}-1\right)\left(\left|\lambda_{2}\right|^{2}-1\right)=0
$$

en exprimant les $\lambda_{i}$ à l'aide de $b$ et $c$ nous obtenons l'égalité $(i)$; quant à $(i i)$ elle est évidente.

L'ensemble $\Sigma \subset \mathbb{C}^{2}$ décrit par (i) et (ii) est un ensemble semi-algébrique connexe dont voici une autre présentation

$$
\begin{aligned}
& \left(|c|^{2}-\frac{1}{2}|b|^{2}+1\right)^{2}-\frac{1}{4}\left|b^{2}-4 c\right|^{2}=0 \\
& |c|^{2}-\frac{1}{2}|b|^{2}+1 \geq 0 \\
& |c|^{2} \leq 1
\end{aligned}
$$

Cette présentation est polynomiale en les parties réelles et imaginaires de $b$ et c. L'ensemble $\Sigma$ est invariant sous l'action de $\mathbb{S}^{1}$ sur $\mathbb{C}^{2}$ donnée par $\left(\mathrm{e}^{\mathrm{i} \vartheta}, b, c\right) \mapsto$ $\left(b \mathrm{e}^{\mathrm{i} \vartheta}, c \mathrm{e}^{2 \mathrm{i} \vartheta}\right)$. Vérifions que $\Sigma$ est LEVI-plat; considérons l'application

$$
\xi: \mathbb{S}^{1} \times \mathbb{C} \rightarrow \mathbb{C}^{2}, \quad\left(\mathrm{e}^{\mathrm{i} \vartheta}+u, \mathrm{e}^{\mathrm{i} \vartheta} u\right) .
$$

L'ensemble décrit par $\left(i_{1}\right)$ et $\left(i_{2}\right)$ est précisément l'image de $\xi$; il est LEvi-plat puisque les droites $u \rightarrow\left(\mathrm{e}^{\mathrm{i} \vartheta}+u, \mathrm{e}^{\mathrm{i} \vartheta} u\right)$ sont contenues dedans. Ces droites font partie d'un 2-tissu linéaire de $\mathbb{C}^{2}$ que nous avons rencontré dans l'Exemple 2.19] : le tissu des tangentes à la parabole $v=u^{2} / 4$. Quant à l'ensemble $\Sigma$ c'est l'image de l'application $\widetilde{\xi}$, restriction de $\xi$ à $\mathbb{S}^{1} \times \overline{\mathbb{D}(0,1)}$ où $\mathbb{D}(0,1)$ est le disque unité de $\mathbb{C}$. Remarquons que $\widetilde{\xi}$ est injective sur $\mathbb{S}^{1} \times \mathbb{D}(0,1)$ et $(2: 1)$ sur le bord $\mathbb{S}^{1} \times \mathbb{S}^{1}$. Par suite $\Sigma$ s'identifie topologiquement à l'espace compact $\mathbb{S}^{1} \times \overline{\mathbb{D}(0,1)}$ où l'on a identifié les points $\left(\mathrm{e}^{\mathrm{i} \vartheta}, \mathrm{e}^{\mathrm{i} \varphi}\right)$ et $\left(\mathrm{e}^{\mathrm{i} \varphi}, \mathrm{e}^{\mathrm{i} \vartheta}\right)$. Son intérieur est un tore plein $\mathbb{S}^{1} \times \mathbb{D}(0,1)$ et son bord $\partial \Sigma$ le quotient du tore $\mathbb{T}^{2}=\mathbb{S}^{1} \times \mathbb{S}^{1}$ par l'involution $(s, t) \mapsto(t, s)$; c'est une bande de MöBius. Nous en déduisons que le bord du bassin d'attraction $\partial \mathrm{W}_{\text {Id }}^{s}(\mathbf{0})$ de $\mathbf{0}$ est l'ensemble semi-algébrique $\operatorname{Inv}^{-1}(\Sigma)$. Il est décrit cette fois par les inéquations $\mathbb{R}$-polynomiales sur $\mathcal{M}(2 ; \mathbb{C})$ :

$$
\left\{\begin{array}{l}
\left(|\operatorname{det}|^{2}-\frac{1}{2}\left|\operatorname{tr}^{2}\right|+1\right)^{2}-\frac{1}{4}\left|\operatorname{tr}^{2}-4 \operatorname{det}\right|^{2}=0 \\
|\operatorname{det}|^{2}-\frac{1}{2}\left|\operatorname{tr}^{2}\right|+1 \geq 0 \\
|\operatorname{det}| \leq 1
\end{array}\right.
$$

Il est LEVI-plat au sens où il contient un ouvert dense (remplacer la dernière inégalité par $\mid$ det $\mid<1)$ LEVI-plat : les variétés complexes contenues dans $\partial \mathrm{W}_{\mathrm{Id}}^{s}(\mathbf{0})$ sont les images réciproques par l'application algébrique $\operatorname{Inv}$ des disques $\mathbb{D}(0,1) \ni$ $u \mapsto\left(\mathrm{e}^{\mathrm{i} \vartheta}+u, \mathrm{e}^{\mathrm{i} \vartheta} u\right), \vartheta$ étant fixé. C'est un ensemble évidemment non borné puisque $\mathrm{W}_{\mathrm{Id}}^{s}(\mathbf{0})$ ne l'est pas (il contient un voisinage des nilpotentes). Ceci va à l'encontre du cas des applications homogènes génériques. Rappelons à cet effet que lorsque $f: \mathbb{C}^{N} \rightarrow \mathbb{C}^{N}$ est une application polynomiale homogène, le bassin d'attraction de 0 est borné dès que $f^{-1}(0)$ se réduit à 0 ce qui est le cas génériquement ([2]). En dimension 1 pour une transformation homogène $z \mapsto z^{k}$ le bord du bassin d'attraction de 0 est évidemment le cercle unité $\mathbb{S}^{1}$ et tous les points périodiques 
sont contenus dans ce cercle (hormis le point 0); de plus ils y sont denses. D'autre part notons que les orbites bornées de $z^{k}$ sont contenues dans la fermeture de ce bassin d'attraction. Dans le contexte de $\Phi_{\text {Id }}$ nous avons la :

Proposition 3.3. Les points périodiques de $\Phi_{\mathrm{Id}}$, excepté $\mathbf{0}$, sont contenus dans le bord $\partial \mathrm{W}_{\mathrm{Id}}^{s}(\mathbf{0})$ du bassin d'attraction de $\mathbf{0}$.

Démonstration. C'est une application directe de la description des points périodiques de $\Phi_{\operatorname{Id} \mid \mathcal{D}}$.

Nous nous intéressons aussi à l'adhérence des points périodiques de $\Phi_{\text {Id }}$ (excepté 0). Cet ensemble a plusieurs composantes. Rappelons les faits élémentaires suivants :

- M a une valeur propre de module 1 et l'autre nulle si et seulement si M est dans l'ensemble

$$
\Lambda^{0}=\{\mathrm{M} \in \mathcal{M}(2 ; \mathbb{C})|\operatorname{det} \mathrm{M}=0,| \operatorname{tr} \mathrm{M} \mid=1\} ;
$$

- M a une valeur propre double de module 1 si et seulement si $\mathrm{M}$ appartient à

$$
\Lambda^{1}=\left\{\mathrm{M} \in \mathcal{M}(2 ; \mathbb{C})\left|\operatorname{trM}^{2}-4 \operatorname{det} \mathrm{M}=0,\right| \operatorname{det} \mathrm{M} \mid=1\right\} ;
$$

- enfin $\mathrm{M}$ a deux valeurs propres de module 1 distinctes si et seulement si $\mathrm{M}$ est dans

$$
\Lambda^{2}=\left\{\mathrm{M} \in \mathcal{M}(2 ; \mathbb{C}) \mid \frac{(\operatorname{trM})^{2}}{\operatorname{det} \mathrm{M}} \in[0,4[,|\operatorname{det} \mathrm{M}|=1\} .\right.
$$

Pour l'application $z \mapsto z^{2}$ l'adhérence des points périodiques coïncide avec le bord du bassin d'attraction de l'origine auquel on ajoute l'origine. L'énoncé qui suit décrit l'adhérence des points périodiques de $\Phi_{\mathrm{Id}}$ et montre via des arguments de dimension que cette adhérence est différente du bord du bassin d'attraction.

Proposition 3.4. L'adhérence des points périodiques de $\Phi_{\mathrm{Id}}$ est l'union de $\{\mathbf{0}\} \cup$ $\Lambda^{0} \cup \Lambda^{1} \cup \Lambda^{2}$.

Démonstration. Le seul point à noter est qu'un élément de $\Lambda^{1}$ est conjugué à une matrice $\left[\begin{array}{cc}\mathrm{e}^{\mathrm{i} \vartheta} & \varepsilon \\ 0 & \mathrm{e}^{\mathrm{i} \vartheta}\end{array}\right]$, avec $\varepsilon \in\{0,1\}$; une telle matrice est limite de matrices diagonalisables $\left[\begin{array}{cc}x_{k_{n}} & \varepsilon \\ 0 & t_{k_{n}}\end{array}\right]$ qui sont périodiques pour $\Phi_{\mathrm{Id}}$.

Cette adhérence est encore un semi-algébrique décrit par l'union disjointe

$$
\{\mathbf{0}\} \sqcup\{|\operatorname{tr}|=1,|\operatorname{det} \mathrm{M}|=0\} \sqcup\left\{\frac{(\operatorname{trM})^{2}}{\operatorname{det} \mathrm{M}} \in[0,4],|\operatorname{det} \mathrm{M}|=1\right\} .
$$

Proposition 3.5. Les orbites bornées de $\Phi_{\mathrm{Id}}$ sont contenues dans $\overline{\mathrm{W}_{\mathrm{Id}}^{s}(\mathbf{0})}$; tout élément $\mathrm{M}$ de $\overline{\mathrm{W}_{\mathrm{Id}}^{s}(\mathbf{0})}$ a son orbite bornée à l'exception des matrices $\mathrm{M}$ ayant pour type de JoRDAN $\left[\begin{array}{ll}\lambda & 1 \\ 0 & \lambda\end{array}\right]$ avec $|\lambda|=1$.

Remarque 3.6. L'image réciproque par Inv du ruban de MöBIUs $\partial \Sigma$ est exactement l'union

$$
\Lambda^{1} \cup \Lambda^{2}=\left\{\frac{(\operatorname{trM})^{2}}{\operatorname{det} M} \in[0,4],|\operatorname{det} M|=1\right\},
$$

l'image réciproque du bord $\partial(\partial \Sigma)$ de cette bande de MöBIUs étant précisément l'ensemble $\Lambda^{1}$. 
Remarque 3.7. Notons que $\Sigma$ et $\partial \Sigma$ sont invariants par Sq $\Phi_{\text {Id }}$ et sont bornés (ceci résulte des présentations (i) et (ii) de la Proposition [3.2).

Problème 1. Il serait intéressant de décrire les automorphismes holomorphes de l'ouvert $\mathrm{W}_{\mathrm{Id}}^{s}(\mathbf{0})$. Parmi ceux-ci on trouve les transformations $\sigma_{\mathrm{P}}: \mathrm{M} \mapsto \mathrm{PMP}^{-1}$ et les transformations linéaires $\Theta: \mathrm{M} \mapsto \mathrm{e}^{\mathrm{i} \vartheta} \mathrm{M}$; dit autrement nous avons une action de $\mathbb{S}^{1} \times \operatorname{PGL}(2 ; \mathbb{C})$. Peut-être serait-il plus facile de décrire les automorphismes holomorphes de $\mathcal{M}(2 ; \mathbb{C}) \simeq \mathbb{C}^{4}$ qui préservent $\mathrm{W}_{\mathrm{Id}}^{s}(\mathbf{0})$ ? De tels automorphismes vont en effet préserver $\partial \mathrm{W}_{\mathrm{Id}}^{s}(\mathbf{0})$ et sa structure LEVI-plate et donc le tissu image réciproque par Inv du tissu des tangentes à la parabole $v=u^{2} / 4$.

3.2. Quelques compléments. Les dynamiques de $\widetilde{\Phi_{\text {Id }}}$ et $\mathbb{P}\left(\Phi_{\text {Id }}\right)$ se déduisent plus ou moins facilement de celle de $\Phi_{\text {Id }}$. La description de $\widetilde{\Phi_{\text {Id }}}$ se fait dès que l'on connait celle de $\Phi_{\text {Id }}: \mathcal{M}(2 ; \mathbb{C}) \rightarrow \mathcal{M}(2 ; \mathbb{C})$ plus celle sur l'hyperplan à l'infini qui est un $\mathbb{P}^{3}(\mathbb{C})$. Mais sur l'hyperplan à l'infini qui est complètement invariant la dynamique est exactement celle de

$$
\mathbb{P}\left(\Phi_{\text {Id }}\right): \mathbb{P}(\mathcal{M}(2 ; \mathbb{C})) \simeq \mathbb{P}^{3}(\mathbb{C}) \rightarrow \mathbb{P}(\mathcal{M}(2 ; \mathbb{C})) \simeq \mathbb{P}^{3}(\mathbb{C}) .
$$

L'application $\mathbb{P}\left(\Phi_{\mathrm{Id}}\right)$ est définie en coordonnées homogènes par

$$
\mathbb{P}\left(\Phi_{\mathrm{Id}}\right):(x: y: z: t) \mapsto\left(x^{2}+y z: y(x+t): z(x+t): y z+t^{2}\right) .
$$

Récapitulons ses propriétés

- le lieu d'indétermination de $\mathbb{P}\left(\Phi_{\text {Id }}\right)$ est le projectivisé de l'ensemble $\mathcal{N}(2 ; \mathbb{C})$ des matrices nilpotentes;

- le projectivisé des matrices de trace nulle (privé de $\mathbb{P}(\mathcal{N}(2 ; \mathbb{C}))$ ) est contracté sur le point $(1: 0: 0: 1)$ correspondant à la matrice identité et ce point est fixe;

- les autres points fixes sont le projectivisé de la quadrique det $=0$, privé de $\mathbb{P}(\mathcal{N}(2 ; \mathbb{C})) ;$

- la fibre générique de $\mathbb{P}\left(\Phi_{\mathrm{Id}}\right)$ a deux éléments ;

- la fibration en 2-plans $\mathcal{P}$ qui est invariante fibre à fibre est maintenant la fibration en $\mathbb{P}^{1}(\mathbb{C})$ radiale en $(1: 0: 0: 1)$ dans $\mathbb{P}^{3}(\mathbb{C})$;

- la droite spéciale $(\lambda, \lambda, 0, \lambda), \lambda \neq 0$, représente à conjugaison près les matrices non diagonalisables (inversibles) et donne un seul point bien sûr dans $\mathbb{P}^{3}(\mathbb{C})$, la «matrice unipotente $\gg(1: 1: 0: 1)$. Nous obtenons l'orbite spéciale $\left(1 / 2^{n}: 1: 0: 1 / 2^{n}\right)$. C'est une orbite discrète qui converge vers $(0: 1: 0: 0)$ qui représente la matrice nilpotente standard. Autrement dit les itérés des matrices unipotentes convergent vers les matrices nilpotentes qui sont d'indétermination;

- la dynamique hormis les problèmes de type points d'indétermination et ensembles contractés se comprend aussi essentiellement via la dynamique de $\chi_{2}: \mathbb{P}^{1}(\mathbb{C}) \rightarrow \mathbb{P}^{1}(\mathbb{C}), z \mapsto z^{2}$. Par exemple dans le $\mathbb{P}^{1}(\mathbb{C})$ des matrices diagonales $\left(\lambda_{1}: 0: 0: \lambda_{2}\right)$ l'application s'écrit $\left(\lambda_{1}^{2}: 0: 0: \lambda_{2}^{2}\right)$ c'est-à-dire $u \mapsto u^{2}$.

Comme pour $\Phi_{\text {Id }}$ on sait décrire la dynamique des polynômes de matrices; par exemple celle de $\varsigma_{c}$ (points périodiques, ensembles invariants) s'obtient essentiellement à partir de $\varsigma_{c_{\mid \mathcal{D}}}$ en faisant agir l'action adjointe; celle de $\varsigma_{c_{\mid \mathcal{D}}}$ se déduit quant à elle directement de celle de $z^{2}+c$. Si on note $\mathcal{J}_{c} \subset \mathbb{C}$ l'ensemble de Julia de $z^{2}+c$, l'ensemble $\mathcal{J}_{c} \times \mathcal{J}_{c} \subset \mathcal{D}$ est invariant par $\varsigma_{c}$. La fermeture de son saturé $\overline{\mathcal{O}\left(\mathcal{J}_{c} \times \mathcal{J}_{c} ; \varsigma_{c}\right)}$ par l'action adjointe est un fermé invariant par $\varsigma_{c}$ dans lequel on a densité des points périodiques. Il y a d'autres fermés invariants, comme par exemple 
le saturé fermé de $\mathcal{J}_{c} \times \mathbb{C} \subset \mathcal{D}$, toujours par l'action adjointe. De même que si $q$ est un point fixe de $z^{2}+c$, alors le saturé fermé de $\mathcal{J}_{c} \times\{q\}$ est encore invariant. La description des orbites bornées est relativement raisonnable (mais zoologique), en liaison avec celles de $z^{2}+c$.

3.3. Centralisateurs. Une façon de mesurer la complexité d'une transformation est d'examiner son centralisateur, i.e. son groupe des commutateurs. La transformation $\chi_{2}$, de même que les $z \mapsto z^{k}$, a un rôle spécial dans la dynamique à une variable; comme les polynômes de TCHEBYCHEV elle a son ensemble de JuLiA lisse et son centralisateur n'est pas réduit à ses propres itérés. Il est donc naturel d'examiner $\ll$ certains centralisateurs $\gg$ de $\Phi_{\text {Id }}$.

Soit $f: X \rightarrow X$ une transformation rationnelle; introduisons les groupes suivants

$$
\begin{aligned}
& \operatorname{Bir}(X ; f)=\{g: X \rightarrow X \text { transformation birationnelle } \mid g \circ f=f \circ g\}, \\
& \operatorname{Aut}(X ; f)=\{g: X \rightarrow X \text { automorphisme holomorphe } \mid g \circ f=f \circ g\} .
\end{aligned}
$$

3.3.1. Caractérisation de $\operatorname{Aut}\left(\mathcal{M}(2 ; \mathbb{C}) ; \Phi_{\mathrm{Id}}\right)$. Les transformations $\sigma_{\mathrm{P}}$ définies par $\sigma_{\mathrm{P}}(\mathrm{M})=\mathrm{PMP}^{-1}$ sont dans $\operatorname{Aut}\left(\mathcal{M}(2 ; \mathbb{C}) ; \Phi_{\mathrm{Id}}\right)$ et sont compatibles à la conjugaison. Notons que si $\Phi$ est compatible à la conjugaison, alors par définition les $\sigma_{\mathrm{P}}$ sont dans $\operatorname{Aut}(\mathcal{M}(2 ; \mathbb{C}) ; \Phi)$. L'application de transposition

$$
\mathcal{T}: \mathcal{M}(2 ; \mathbb{C}) \rightarrow \mathcal{M}(2 ; \mathbb{C}), \quad\left[\begin{array}{cc}
x & y \\
z & t
\end{array}\right] \mapsto\left[\begin{array}{cc}
x & z \\
y & t
\end{array}\right]
$$

commute à $\Phi_{\text {Id }} ;$ ainsi $\operatorname{Aut}\left(\mathcal{M}(2 ; \mathbb{C}) ; \Phi_{\text {Id }}\right)$ contient des éléments non compatibles à la conjugaison bien que $\Phi_{\text {Id }}$ le soit. De même la transformation

$$
\mathcal{I}: \mathcal{M}(2 ; \mathbb{C}) \rightarrow \mathcal{M}(2 ; \mathbb{C}), \quad\left[\begin{array}{cc}
x & y \\
z & t
\end{array}\right] \mapsto\left[\begin{array}{cc}
t & y \\
z & x
\end{array}\right]
$$

commute à $\Phi_{\text {Id }}$ et n'est pas compatible à la conjugaison; notons que $\mathcal{I}(\mathrm{M})=$ ${ }^{\mathrm{t}}\left(\sigma_{\mathrm{P}}(\mathrm{M})\right)$ où $\mathrm{P}=\left[\begin{array}{ll}0 & 1 \\ 1 & 0\end{array}\right]$.

Proposition 3.8. Le groupe $\operatorname{Aut}\left(\mathcal{M}(2 ; \mathbb{C}) ; \Phi_{\mathrm{Id}}\right)$ est engendré par la transposition $\mathcal{T}$ et les $\sigma_{\mathrm{P}} ;$ plus précisément

$$
\operatorname{Aut}\left(\mathcal{M}(2 ; \mathbb{C}) ; \Phi_{\text {Id }}\right) \simeq \operatorname{PGL}(2 ; \mathbb{C}) \rtimes \mathbb{Z} / 2 \mathbb{Z} .
$$

Démonstration. Soit $\varphi$ un automorphisme de $\mathcal{M}(2 ; \mathbb{C})$ qui commute à $\Phi_{\text {Id }}$. Puisque 0 est le seul point fixe de $\Phi_{\text {Id }}$ où la différentielle de $\Phi_{\text {Id }}$ est identiquement nulle, 0 est fixe $\operatorname{par} \varphi$. On écrit $\varphi=\varphi_{1}+\varphi_{2}+\ldots$ le développement de TAYLOR de $\varphi$, chaque $\varphi_{k}$ étant holomorphe de degré $k$. Notons que la partie linéaire $\varphi_{1}$ de $\varphi$ est dans $\operatorname{Aut}\left(\mathcal{M}(2 ; \mathbb{C}) ; \Phi_{\text {Id }}\right)$ de sorte que $\Psi=\varphi \circ \varphi_{1}^{-1}$ aussi. Supposons que $\Psi$ ne soit pas trivial ; écrivons $\Psi$ sous la forme $\Psi=\mathrm{Id}+\Psi_{k}+\Psi_{k+1}+\ldots$ avec $\Psi_{k}$ homogène de degré $k>1$ et $\Psi_{k}$ non nul. La commutation de $\Psi$ et $\Phi_{\text {Id }}$ implique l'égalité suivante

$$
\begin{aligned}
\mathrm{M}^{2}+\Psi_{k}\left(\mathrm{M}^{2}\right)+\Psi_{k+1}\left(\mathrm{M}^{2}\right)+\ldots & =\left(\mathrm{M}+\Psi_{k}(\mathrm{M})+\Psi_{k+1}(\mathrm{M})+\ldots\right)^{2} \\
& =\mathrm{M}^{2}+\mathrm{M} \Psi_{k}(\mathrm{M})+\Psi_{k}(\mathrm{M}) \mathrm{M}+\ldots
\end{aligned}
$$

Pour des raisons de degré nous avons pour tout M l'égalité $\mathrm{M} \Psi_{k}(\mathrm{M})+\Psi_{k}(\mathrm{M}) \mathrm{M}=0$. Écrivons M (resp. $\left.\Psi_{k}(\mathrm{M})\right)$ sous la forme $\left[\begin{array}{ll}x & y \\ z & t\end{array}\right]\left(\operatorname{resp} .\left[\begin{array}{cc}X(\mathrm{M}) & Y(\mathrm{M}) \\ Z(\mathrm{M}) & T(\mathrm{M})\end{array}\right]\right)$; alors 
$\mathrm{M} \Psi_{k}(\mathrm{M})+\Psi_{k}(\mathrm{M}) \mathrm{M}=0$ se réécrit

$$
\left\{\begin{array}{l}
2 x X+y Z+z Y=0 \\
x Y+y T+y X+t Y=0, \\
z X+t Z+x Z+z T=0, \\
z Y+y Z+2 t T=0
\end{array}\right.
$$

On peut voir (3.1) comme un système linéaire, les inconnues étant les $X, Y, Z$, $T$, dont on notera $\Delta=\Delta(\mathrm{M})$ le déterminant. Nous constatons que $\Delta(\mathrm{Id})=16$; par suite $\Delta$ est non identiquement nul. En résulte que $X, Y, Z, T$, et donc $\Psi_{k}$, sont identiquement nuls. Nous en déduisons que $\varphi=\varphi_{1}$ est nécessairement un automorphisme linéaire.

Remarquons que Id est l'unique point fixe de $\Phi_{\text {Id }}$ en lequel la différentielle de $\Phi_{\text {Id }}$ est 2 Id. Il s'en suit que Id est fixé par $\varphi_{1}$ et la droite $\mathcal{C}=\{\lambda \operatorname{Id} \mid \lambda \in \mathbb{C}\}$ est invariante point par point $\operatorname{par} \varphi_{1}$. En particulier $\varphi_{1}$ envoie le plan $\langle\lambda \mathrm{Id}+\mu \mathrm{M}\rangle$ dans le plan $\langle\lambda \mathrm{Id}+\mu \varphi(\mathrm{M})\rangle$. Ceci implique que la fibration $\mathcal{P}$ est invariante par $\varphi_{1}$. Pour $\mathrm{M}$ générique, $\mathrm{M}$ et $\varphi_{1}(\mathrm{M})$ sont diagonalisables : $\mathrm{M}=\mathrm{QDQ}^{-1}, \varphi_{1}(\mathrm{M})=\mathrm{Q}^{\prime} \mathrm{D}^{\prime} \mathrm{Q}^{\prime-1}$. En composant $\varphi_{1}$ à gauche et à droite par des $\sigma_{\mathrm{P}}$ ad-hoc nous nous ramenons donc au cas où $\varphi_{1}$ respecte la fibration $\mathcal{P}$ et le groupe $\mathcal{D}$. Si on écrit $\varphi(\mathrm{M})$ sous la forme $\left[\begin{array}{cc}X(\mathrm{M}) & Y(\mathrm{M}) \\ Z(\mathrm{M}) & T(\mathrm{M})\end{array}\right]$ où cette fois les transformations $X, Y, Z$ et $T$ sont linéaires

$$
\begin{gathered}
Y(x, 0,0, t)=Z(x, 0,0, t)=0, \quad X\left(x^{2}, 0,0, t^{2}\right)=X^{2}(x, 0,0, t), \\
T\left(x^{2}, 0,0, t^{2}\right)=T^{2}(x, 0,0, t) .
\end{gathered}
$$

Puisque $\varphi_{1}$ est un automorphisme, ceci entraîne par un calcul direct l'alternative suivante :

$$
\begin{aligned}
& \text { ou bien } X(x, 0,0, t)=x \text { et } T(x, 0,0, t)=t ; \\
& \text { ou bien } X(x, 0,0, t)=t \text { et } T(x, 0,0, t)=x .
\end{aligned}
$$

Quitte à composer $\varphi_{1}$ par l'involution $\mathcal{I}$, nous pouvons supposer que nous sommes dans la première situation. Finalement

$$
\varphi_{1}=\left(x+\ell_{1}(y, z), \ell_{2}(y, z), \ell_{3}(y, z), t+\ell_{4}(y, z)\right)
$$

les $\ell_{i}$ étant linéaires en $y, z$. En réécrivant que $\Phi_{\text {Id }}$ et $\varphi_{1}$ commutent on constate aisément que $\ell_{1}=\ell_{4}=0$ et $\ell_{2} \ell_{3}=y z$. Ainsi après ces modifications $\varphi_{1}$ est de l'un des deux types suivants

$$
g=\left(x, \rho y, \frac{z}{\rho}, t\right), \quad h=\left(x, \rho z, \frac{y}{\rho}, t\right) .
$$

Remarquons que si $\mathrm{P}=\left[\begin{array}{cc}\sqrt{\rho} & 0 \\ 0 & \rho\end{array}\right]$, alors $g=\sigma_{\mathrm{P}}$ et que, modulo $\sigma_{\mathrm{P}}$, l'application $h$ est la transposition.

3.3.2. Description de $\operatorname{Bir}\left(\mathbb{P} \mathcal{M}(2 ; \mathbb{C}) ; \mathbb{P}\left(\Phi_{\text {Id }}\right)\right)$. Dans la carte $t=1$ on remarque que $\mathbb{P}\left(\Phi_{\text {Id }}\right)$ s'écrit de la façon suivante

$$
\left(\frac{x^{2}+y z}{1+y z}, \frac{y(x+1)}{1+y z}, \frac{z(x+1)}{1+y z}\right) .
$$

La fibration en 2-plans $\mathcal{P}$, invariante par $\Phi_{\text {Id }}$, initialement donnée par les niveaux de $\left(\frac{y}{z}, \frac{x-t}{z}\right)$, produit maintenant la fibration en droites donnée cette fois par les 
fibres de $\left(\frac{y}{z}, \frac{x-1}{z}\right)$. Nous allons trivialiser cette fibration en conjuguant $\mathbb{P}\left(\Phi_{\text {Id }}\right)$ par la transformation birationnelle $f:(x, y, z) \mapsto(x z+1, y z, z)$. On vérifie que

$$
G=f^{-1} \mathbb{P}\left(\Phi_{\text {Id }}\right) f=\left(x, y, \frac{z(2+x z)}{1+y z^{2}}\right) .
$$

Le fait que $\mathcal{P}$ soit l'unique fibration en surfaces invariante fibre à fibre par $\Phi_{\text {Id }}$ implique que la fibration en droites $(x, y)=$ cte est l'unique fibration en courbes préservée par $G$. Il en résulte que si $F$ est une application birationnelle commutant à $G$, alors $F$ est nécessairement du type

$$
\left(X(x, y), Y(x, y), \frac{a(x, y) z+b(x, y)}{c(x, y) z+d(x, y)}\right), \quad a, b, c, d \in \mathbb{C}\{x, y\}, a d-b c \neq \equiv 0 .
$$

Visiblement la transformation $(x, y) \rightarrow(X(x, y), Y(x, y))$ doit être birationnelle. Si l'on considère $F$ et $G$ comme des applications rationnelles sur $\mathbb{C}^{2} \times \mathbb{P}^{1}$ on constate qu'elles sont fibrées et holomorphes en restriction aux fibres génériques. Les points fixes de $G$ sont les trois surfaces irréductibles $z=0, z=\infty$ et $y z^{2}-x z-1=0$. Si $F$ commute à $G$, alors $F$ préserve dans leur ensemble les points fixes de $G$. Plus précisément $F$ laisse invariante la surface $y z^{2}-x z-1=0$ et fixe ou permute les plans $z=0$ et $z=\infty$.

(i) Supposons, dans un premier temps, que $y z^{2}-x z-1=0, z=0$ et $z=\infty$ soient fixés par $F$. Alors $F$ est du type suivant

$$
F(x, y, z)=(X(x, y), Y(x, y), a(x, y) z) .
$$

Maintenant $\left(y z^{2}-x z-1\right) \circ F=Y a^{2} z^{2}-X a z-1$ est donc un multiple (comme élément de $\mathbb{C}(x, y)[z])$ de $y z^{2}-x z-1$, de sorte que nous pouvons préciser $F$ :

$$
F(x, y, z)=\left(\frac{x}{a(x, y)}, \frac{y}{a^{2}(x, y)}, a(x, y) z\right) .
$$

On peut d'ailleurs remarquer que toute transformation rationnelle comme ci dessus, i.e. pour tout choix de $a$, commute à $G$. Le fait que $F$ soit birationnelle impose des restrictions sur $a$. En effet si $F$ est birationnelle alors $H:(x, y) \mapsto\left(\frac{x}{a(x, y)}, \frac{y}{a^{2}(x, y)}\right)$ l'est aussi. En conjuguant $H$ par $\left(x, x^{2} y\right)$ on constate que $\left(\frac{x}{a\left(x, x^{2} y\right)}, y\right)$ est birationnelle ce qui force $\frac{x}{a\left(x, x^{2} y\right)}$ à être une transformation de MöBIus en $x$ à paramètre $y$. Nous en déduisons que

$$
a(x, y)=x \frac{\alpha\left(\frac{y}{x^{2}}\right) x+\beta\left(\frac{y}{x^{2}}\right)}{\gamma\left(\frac{y}{x^{2}}\right) x+\delta\left(\frac{y}{x^{2}}\right)}
$$

où les $\alpha, \beta, \gamma, \delta$ sont dans $\mathbb{C}(t)$ et $\alpha \delta-\beta \gamma \neq 0$. Le groupe des transformations birationnelles

$$
\left\{\left(\frac{x}{a(x, y)}, \frac{y}{a^{2}(x, y)}, a(x, y) z\right) \mid \text { avec } a \text { du type (3.2) }\right\}
$$

commute à $G$ et est isomorphe à $\operatorname{PGL}(2 ; \mathbb{C}(t))$.

(ii) Maintenant considérons l'éventualité où $F$ fixe $y z^{2}-x z-1$ et permute les plans $z=0$ et $z=\infty$. Alors $F$ est du type $\left(X(x, y), Y(x, y), \frac{a(x, y)}{z}\right)$. Comme $\left(y z^{2}-\right.$ $x z-1) \circ F=\frac{Y a^{2}-X a z-z^{2}}{z^{2}}$ nous obtenons par le même argument que précédemment

$$
F(x, y, z)=\left(-\frac{x}{a(x, y) y}, \frac{1}{a^{2}(x, y) y}, \frac{a(x, y)}{z}\right) ;
$$


un tel $F$ ne commute jamais à $G$. Nous pouvons donc énoncer la :

Proposition 3.9. On $a$ :

$$
\begin{aligned}
\operatorname{Bir}\left(\mathbb{P} \mathcal{M}(2 ; \mathbb{C}) ; \mathbb{P}\left(\Phi_{\mathrm{Id}}\right)\right)=\{ & f\left(\frac{x}{a(x, y)}, \frac{y}{a^{2}(x, y)}, a(x, y) z\right) f^{-1} \mid \\
& \text { avec a du type (3.2) }, f=(x z+1, y z, z)\} ;
\end{aligned}
$$

en particulier $\operatorname{Bir}\left(\mathbb{P} \mathcal{M}(2 ; \mathbb{C}) ; \mathbb{P}\left(\Phi_{\mathrm{Id}}\right)\right)$ est isomorphe à $\mathrm{PGL}(2 ; \mathbb{C}(t))$.

3.3.3. Le groupe $\operatorname{Bir}\left(\mathcal{M}(2 ; \mathbb{C}) ; \Phi_{\mathrm{Id}}\right)$. Donnons un exemple de transformation appartenant à $\operatorname{Bir}\left(\mathcal{M}(2 ; \mathbb{C}) ; \Phi_{\mathrm{Id}}\right)$. L'application

$$
\iota: \mathcal{M}(2 ; \mathbb{C}) \rightarrow \mathcal{M}(2 ; \mathbb{C}), \quad \mathrm{M} \mapsto \mathrm{M}^{-1}
$$

est dans $\operatorname{Bir}\left(\mathcal{M}(2 ; \mathbb{C}) ; \Phi_{\text {Id }}\right)$; elle est compatible à la conjugaison. Remarquons qu'elle commute aux applications $\sigma_{\mathrm{P}}$ ainsi qu'à $\mathcal{T}$. On constate que la restriction de $\iota$ aux matrices diagonales s'identifie à l'involution de CREMONA standard en dimension $\operatorname{deux}(x, t) \rightarrow\left(\frac{1}{x}, \frac{1}{t}\right)$.

Soit $f$ un élément de $\operatorname{Bir}\left(\mathcal{M}(2 ; \mathbb{C}) ; \Phi_{\mathrm{Id}}\right)$. Comme $f\left(\mathrm{M}^{2}\right)=f(\mathrm{M}) f(\mathrm{M})$ nous avons $\operatorname{det} f\left(\mathrm{M}^{2}\right)=(\operatorname{det} f(\mathrm{M}))^{2}$. Ce type de propriété est décrit dans le lemme qui suit.

Lemme 3.10. Soit $\psi: \mathcal{M}(2 ; \mathbb{C}) \rightarrow \mathbb{P}^{1}(\mathbb{C})$ une fonction rationnelle satisfaisant l'équation fonctionnelle $\psi\left(\mathrm{M}^{2}\right)=\psi(\mathrm{M})^{2}$ pour tout $\mathrm{M}$ dans $\mathcal{M}(2 ; \mathbb{C})$.

Alors $\psi$ est de la forme $\mathrm{M} \rightarrow \varepsilon(\operatorname{det} \mathrm{M})^{k}$ où $k$ désigne un entier relatif et $\varepsilon$ un élément de $\{0,1\}$.

Démonstration. Quitte à changer $\psi$ en $1 / \psi$ nous pouvons supposer que l'hyperplan $\mathfrak{s l}(2 ; \mathbb{C})$ n'est pas contenu dans les pôles de $\psi$. Ainsi $\psi$ est rationnelle en restriction à $\mathfrak{s l}(2 ; \mathbb{C})$ et pour $x, y, z$ génériques $\psi(x, y, z,-x)$ est bien défini. Par suite $\psi\left(\left(x^{2}+y z\right) \mathrm{Id}\right)$ est rationnel, bien défini et donc $\delta: t \mapsto \psi(t \mathrm{Id})$ aussi. L'hypothèse $\psi\left(\mathrm{M}^{2}\right)=\psi(\mathrm{M})^{2}$ implique que $\delta\left(t^{2}\right)=(\delta(t))^{2}$, i.e. l'application $\delta$ commute à $s \mapsto s^{2}$; par conséquent $\delta(t)=\varepsilon^{\prime} t^{k^{\prime}}$ pour un certain $k^{\prime}$ dans $\mathbb{Z}$ et pour $\varepsilon^{\prime}$ dans $\{0,1\}$. Autrement dit pour tout $\mathrm{M}$ dans $\mathfrak{s l}(2 ; \mathbb{C})$ nous avons $(\psi(\mathrm{M}))^{2}=\psi\left(\mathrm{M}^{2}\right)=$ $\psi(\operatorname{det}(\mathrm{M}) \mathrm{Id})=\varepsilon^{\prime}(\operatorname{det} \mathrm{M})^{k^{\prime}}$. La restriction de $\Psi$ à $\mathfrak{s l}(2 ; \mathbb{C})$ est donc donnée par $\psi(\mathrm{M})=\varepsilon(\operatorname{det} \mathrm{M})^{k}$ pour un certain $k$ dans $\mathbb{Z}$ et pour $\varepsilon$ dans $\{0,1\}$.

Soit $\mathrm{M}$ dans $\mathcal{M}(2 ; \mathbb{C})$ tel que $\mathrm{M}^{2}$ appartienne à $\mathfrak{s l}(2 ; \mathbb{C})$. D'une part $\psi\left(\mathrm{M}^{2}\right)=$ $\varepsilon(\operatorname{det} \mathrm{M})^{2 k}$ et d'autre part $\psi\left(\mathrm{M}^{2}\right)=(\psi(\mathrm{M}))^{2}$; nous en déduisons que $\psi(\mathrm{M})$ s'écrit aussi $\varepsilon(\operatorname{det} M)^{k}$. On peut répéter cet argument avec tous les éléments $\mathrm{M}$ de $\mathcal{M}(2 ; \mathbb{C})$ pour lesquels $\mathrm{M}^{2^{n}}$ appartient à $\mathfrak{s l}(2 ; \mathbb{C})$ pour un certain $n$. L'ensemble de ces matrices est ZARISKI dense d'où l'énoncé.

Soit $f$ dans $\operatorname{Bir}\left(\mathcal{M}(2 ; \mathbb{C}) ; \Phi_{\text {Id }}\right)$; on peut appliquer le Lemme 3.10 à $\psi=\operatorname{det} f$ : il existe $k$ tel que $\operatorname{det} f(\mathrm{M})=\varepsilon(\operatorname{det} \mathrm{M})^{k}, \varepsilon \in\{0,1\}$. Comme $f$ est birationnelle $\varepsilon$ ne peut être nul et la fibration det $=$ cte est invariante sous l'action de $f$. Nécessairement ceci implique que $k= \pm 1$ et, quitte à composer $f$ par $\iota: \mathrm{M} \rightarrow \mathrm{M}^{-1}$, nous supposerons dans la suite que $k=1$, i.e. $\operatorname{det} f(\mathrm{M})=\operatorname{det} \mathrm{M}$.

Supposons que $f \in \operatorname{Bir}\left(\mathcal{M}(2 ; \mathbb{C}) ; \Phi_{\mathrm{Id}}\right)$ ait un pôle, i.e. $f$ s'écrit $\frac{g}{h}$ avec $g, h$ polynomiales et $h$ non constante. Nous pouvons bien évidemment nous ramener au cas où $g$ et $h$ n'ont pas de facteur commun. 
Lemme 3.11. L'hypersurface $h=0$ est complètement invariante.

Démonstration. Plaçons-nous dans $\mathbb{P}^{4}(\mathbb{C})$; notons $\widetilde{\Phi_{\text {Id }}}($ resp. $\widetilde{f})$ le prolongement de $\Phi_{\text {Id }}$ (resp. $f$ ) à $\mathbb{P}^{4}(\mathbb{C})$. Désignons l'hyperplan à l'infini par $\mathcal{H}_{\infty}$. Puisque $\widehat{\Phi_{\mathrm{Id}}}(x: y: z: t: s)=\left(x^{2}+y z: y(x+t): z(x+t): t^{2}+y z: s^{2}\right)$ l'hyperplan $\mathcal{H}_{\infty}$ est complètement invariant par $\widetilde{\Phi_{\text {Id }}}$.

Soit M un point générique de $h=0$. D'une part M n'est pas d'indétermination pour $\widetilde{f}$, d'autre part $\widetilde{f}(\mathrm{M})$ appartient à $\mathcal{H}_{\infty}$; la complète invariance de $\mathcal{H}_{\infty}$ assure que $\widetilde{\Phi_{\mathrm{Id}}}(\widetilde{f}(\mathrm{M}))$ aussi. Comme $\widetilde{f}$ et $\widetilde{\Phi_{\mathrm{Id}}}$ commutent, $\widetilde{f}\left(\widetilde{\Phi_{\mathrm{Id}}}(\mathrm{M})\right)$ est sur $\mathcal{H}_{\infty}$; il s'en suit que $\widetilde{\Phi_{\text {Id }}}(\mathrm{M})$ appartient à $h=0$.

Par ailleurs soit $\mathrm{M}$ tel que $\widetilde{\Phi_{\mathrm{Id}}}(\mathrm{M})$ soit contenu dans $h=0$, M générique pour cette propriété; alors $\widetilde{f}\left(\widetilde{\Phi_{\mathrm{Id}}}(\mathrm{M})\right)$ appartient à $\mathcal{H}_{\infty}$ et, par commutation de $\widetilde{f}$ et $\widetilde{\Phi_{\mathrm{Id}}}$, $\widetilde{\Phi_{\text {Id }}}(\widetilde{f}(\mathrm{M}))$ aussi. La complète invariance de $\mathcal{H}_{\infty}$ par $\widetilde{\Phi_{\text {Id }}}$ assure que $\widetilde{f}(\mathrm{M})$ appartient à $\mathcal{H}_{\infty}$.

Le Lemme 3.11 assure que l'hypersurface $h=0$ est complètement invariante par $\Phi_{\text {Id }}$. D'après [3] il existe une constante non nulle $c$ telle que $h\left(\mathrm{M}^{2}\right)=c(h(\mathrm{M}))^{2}$. Mais quitte à changer $h$ en $h / c$ nous pouvons supposer dans la suite que $h\left(\mathrm{M}^{2}\right)=$ $h(\mathrm{M})^{2}$. En particulier il existe un certain entier $s \in \mathbb{N}$ tel que, pour tout $\mathrm{M}$ dans $\mathcal{M}(2 ; \mathbb{C})$, nous ayons $h(\mathrm{M})=(\operatorname{det} \mathrm{M})^{s}$.

En considérant l'adhérence de ZARISKI de l'orbite d'une matrice générique $\mathrm{M}$ on constate que $f$ laisse invariante la fibration en 2-plans $\mathcal{P}$, i.e. $\mathcal{P}(f(\mathrm{M}))=f(\mathcal{P}(\mathrm{M}))$. Quitte à changer $f$ en $\sigma_{\mathrm{P}} f \sigma_{\mathrm{Q}}$ avec $\mathrm{P}, \mathrm{Q}$ bien choisis nous pouvons supposer que $f$ est rationnelle et bien définie sur $\mathcal{D}$ et que $\mathcal{D}$ est invariant par $f$. Ceci signifie que la restriction de $f$ à $\mathcal{D}$ induit une application birationnelle $f_{\mid \mathcal{D}}: \mathcal{D} \rightarrow \mathcal{D}$. Évidemment $f_{\mid \mathcal{D}}$ commute à la restriction de $\Phi_{\text {Id }}$ à $\mathcal{D}$. Le lemme qui suit se démontre facilement.

Lemme 3.12. Soit $\eta: \mathbb{C}^{2} \rightarrow \mathbb{C}^{2}$ une transformation birationnelle commutant $\grave{a}$ $\xi: \mathbb{C}^{2} \rightarrow \mathbb{C}^{2},(x, t) \mapsto\left(x^{2}, t^{2}\right)$. Alors $\eta$ est de l'un des types suivants

$$
(x, t), \quad(t, x), \quad\left(\frac{1}{x}, \frac{1}{t}\right), \quad\left(\frac{1}{t}, \frac{1}{x}\right), \quad\left(x, \frac{1}{t}\right), \quad\left(\frac{1}{x}, t\right), \quad\left(\frac{1}{t}, x\right), \quad\left(t, \frac{1}{x}\right) .
$$

Autrement dit $\operatorname{Bir}\left(\mathbb{C}^{2} ; \xi\right)$ est engendré par les involutions $\left(\frac{1}{x}, t\right)$ et $(t, x)$.

L'énoncé 3.12 s'applique évidemment à $f_{\mid \mathcal{D}}$ puisque $\Phi_{\operatorname{Id}_{\mid \mathcal{D}}}$ s'identifie à $(x, t) \mapsto$ $\left(x^{2}, t^{2}\right)$. Mais nous avons supposé que $\operatorname{det} f(\mathrm{M})=\operatorname{det} \mathrm{M}$, ce qui n'autorise que les deux premiers modèles du Lemme 3.12; nous en déduisons, après les adaptations précédentes, que l'application $f_{\mid \mathcal{D}}$ est soit l'identité, soit $\operatorname{diag}(x, t) \mapsto \operatorname{diag}(t, x)$. Mais quitte à modifier encore $f$ en composant par $\mathcal{I}:\left[\begin{array}{ll}x & y \\ z & t\end{array}\right] \rightarrow\left[\begin{array}{cc}t & y \\ z & x\end{array}\right]$ nous nous ramenons à la première éventualité, i.e. à $f_{\mid \mathcal{D}}=\mathrm{id}_{\mid \mathcal{D}}$. Récapitulons, nous pouvons supposer que

$-\operatorname{det} f(\mathrm{M})=\operatorname{det} \mathrm{M}$;

$-f_{\mid \mathcal{D}}=\mathrm{id}_{\mid \mathcal{D}}$

- si $f$ a un pôle alors $f$ s'écrit $f(\mathrm{M})=\frac{g(\mathrm{M})}{(\operatorname{det} \mathrm{M})^{s}}$.

On constate que $f$ est alors définie holomorphe au voisinage de l'identité qui est un point fixe de $\Phi_{\text {Id }}$ et de $f$. La partie linéaire de $\Phi_{\text {Id }}$ au point fixe Id est la multiplication par 2. En fait l'application exponentielle exp: $\mathcal{M}(2 ; \mathbb{C}) \rightarrow \mathcal{M}(2 ; \mathbb{C})$ 
linéarise l'application $\Phi_{\mathrm{Id}}$,

$$
\Phi_{\text {Id }}(\exp M)=(\exp M)^{2}=\exp (2 \mathrm{M}) .
$$

Maintenant les seules transformations holomorphes qui commutent à $\mathrm{M} \mapsto 2 \mathrm{M}$ sont les transformations linéaires. Ceci implique que si $f$ satisfait les propriétés qui précèdent, exp linéarise $f$ au voisinage de l'identité

$$
f(\exp \mathrm{M})=\exp f_{1}(\mathrm{M})
$$

où $f_{1}=D f_{(\mathrm{Id})}$ est la partie linéaire de $f$ en Id. Notons que l'application $\mathrm{M} \mapsto$ $f(\exp \mathrm{M})$ est partout définie holomorphe puisque

$$
f(\exp \mathrm{M})=\frac{g(\exp \mathrm{M})}{(\operatorname{det} \exp \mathrm{M})^{s}}=\frac{g(\exp \mathrm{M})}{(\exp \operatorname{tr} \mathrm{M})^{s}} .
$$

Il s'en suit que $f_{1}$ détermine $f$ et que l'identité (3.3) est en fait globale.

Nous allons maintenant décrire $f_{1}$ en utilisant les propriétés de $f$. Puisque $f$ est l'identité sur $\mathcal{D}$, nous avons

$$
f_{1}(x, y, z, t)=\left(x+a_{1} y+b_{1} z, a_{2} y+b_{2} z, a_{3} y+b_{3} z, t+a_{4} y+b_{4} z\right) .
$$

Comme det est invariant par $f$ et que infinitésimalement le déterminant en Id s'identifie à la trace (via exp), l'application $f_{1}$ préserve la trace c'est-à-dire $a_{1}+a_{4}=$ $b_{1}+b_{4}=0$. Soient $\mathrm{P}$ une matrice inversible et $p, q$ dans $\mathbb{Z}$; on a

$$
\mathrm{Id}=\mathrm{P} \cdot \exp (\operatorname{diag}(2 \mathrm{i} \pi p, 2 \mathrm{i} \pi q)) \cdot \mathrm{P}^{-1}=\exp \left(\mathrm{P} \cdot \operatorname{diag}(2 \mathrm{i} \pi p, 2 \mathrm{i} \pi q) \cdot \mathrm{P}^{-1}\right)
$$

et en utilisant (3.3),

$$
\begin{aligned}
\mathrm{Id}=f(\mathrm{Id}) & =f\left(\exp \left(\mathrm{P} \cdot \operatorname{diag}(2 \mathrm{i} \pi p, 2 \mathrm{i} \pi q) \cdot \mathrm{P}^{-1}\right)\right) \\
& =\exp \left(f_{1}\left(\mathrm{P} \cdot \operatorname{diag}(2 \mathrm{i} \pi p, 2 \mathrm{i} \pi q) \cdot \mathrm{P}^{-1}\right)\right) .
\end{aligned}
$$

En particulier $f_{1}\left(\mathrm{P} \cdot \operatorname{diag}(2 \mathrm{i} \pi p, 2 \mathrm{i} \pi q) \cdot \mathrm{P}^{-1}\right)$ est un conjugué d'un certain $\operatorname{diag}\left(2 \mathrm{i} \pi p^{\prime}, 2 \mathrm{i} \pi q^{\prime}\right)$. Ceci signifie qu'il y a un ensemble dénombrable d'orbites de l'action adjointe qui sont envoyées par $f_{1}$ sur d'autres orbites de cette même action. Par passage à l'adhérence de ZARISKI nous en déduisons que $f_{1}$ envoie orbites (de l'action adjointe) dans orbites. En particulier la fibration par Inv $=($ tr, det) est invariante $\operatorname{par} f_{1}$. Soit $Q$ la forme quadratique $Q=\operatorname{det} f_{1}$ :

$$
Q=\left(x+a_{1} y+b_{1} z\right)\left(t-a_{1} y-b_{1} z\right)-\left(a_{2} y+b_{2} z\right)\left(a_{3} y+b_{3} z\right) .
$$

Puisque $Q$ doit être constant sur les niveaux de Inv nous obtenons en utilisant un argument de 8

$$
Q=\varphi(\operatorname{tr}, \operatorname{det})
$$

avec $\varphi$ un germe d'application holomorphe en 0, l'égalité (3.4) étant comprise au voisinage de 0 . Mais en développant $\varphi$ en série et en utilisant l'expression de $Q$ nous constatons que $Q=\operatorname{det}$ et par suite $a_{1}=b_{1}=0$ et $\left[\begin{array}{ll}a_{2} & b_{2} \\ a_{3} & b_{3}\end{array}\right]$ est l'une des matrices Id ou $\left[\begin{array}{ll}0 & 1 \\ 1 & 0\end{array}\right]$. Dans le premier cas $f_{1}=$ Id et donc $f(M)=M$; le second cas montre que $f_{1}$ est la différentielle de l'application transposition, et par conséquent $f$ coïncide elle-même avec cette transposition (nous utilisons la commutation de $\Phi_{\text {Id }}$ avec $\mathcal{T}$ et le fait que la partie linéaire $f_{1}$ détermine $f$ ). Finalement 
nous avons la

Proposition 3.13. Le groupe $\operatorname{Bir}\left(\mathcal{M}(2 ; \mathbb{C}) ; \Phi_{\text {Id }}\right)$ est engendré par $\operatorname{Aut}\left(\mathcal{M}(2 ; \mathbb{C}) ; \Phi_{\text {Id }}\right)$ $\simeq \operatorname{PGL}(2 ; \mathbb{C}) \rtimes \mathbb{Z} / 2 \mathbb{Z}$ et par l'involution $\iota: \mathrm{M} \mapsto \mathrm{M}^{-1}$.

Remarque 3.14. D'un point de vue abstrait $\operatorname{Bir}\left(\mathcal{M}(2 ; \mathbb{C}) ; \Phi_{\text {Id }}\right)$ s'identifie à $(\operatorname{PGL}(2 ; \mathbb{C}) \rtimes \mathbb{Z} / 2 \mathbb{Z}) \times \mathbb{Z} / 2 \mathbb{Z}$.

Remarque 3.15. L'ensemble des transformations rationnelles qui commutent à $\Phi_{\text {Id }}$ contient $\operatorname{Bir}\left(\mathcal{M}(2 ; \mathbb{C}) ; \Phi_{\text {Id }}\right)$ mais aussi les transformations suivantes

$$
\mathrm{M} \mapsto \mathrm{M}^{k}, \quad \mathrm{M} \mapsto(\operatorname{det} \mathrm{M})^{k} \mathrm{Id}, \quad \mathrm{M} \mapsto\left(\operatorname{det} \mathrm{M}^{k}\right)\left[\begin{array}{ll}
1 & 0 \\
0 & 0
\end{array}\right], \quad k \in \mathbb{Z}
$$

Remarque 3.16. On vérifie que si $\Phi$ appartient à $\operatorname{Bir}\left(\mathcal{M}(2 ; \mathbb{C}) ; \Phi_{\text {Id }}\right)$, alors $\Phi$ commute à toutes les transformations $\Phi_{k}: \mathrm{M} \mapsto \mathrm{M}^{k}$, autrement dit on a les inclusions

$$
\operatorname{Bir}\left(\mathcal{M}(2 ; \mathbb{C}) ; \Phi_{\text {Id }}\right) \subset \operatorname{Bir}\left(\mathcal{M}(2 ; \mathbb{C}) ; \Phi_{k}\right), k \geq 2 .
$$

Ces inclusions ne sont pas des égalités : $\mathrm{M} \mapsto-\mathrm{M}$ commute à $\Phi_{3}$ mais pas à $\Phi_{\mathrm{Id}}$.

Remarque 3.17. Soit $\Phi: \mathcal{M}(2 ; \mathbb{C}) \rightarrow \mathcal{M}(2 ; \mathbb{C})$ une application compatible à la multiplication : $\Phi\left(\mathrm{M}_{1} \mathrm{M}_{2}\right)=\Phi\left(\mathrm{M}_{1}\right) \Phi\left(\mathrm{M}_{2}\right)$. Un tel $\Phi$ commute à $\Phi_{\text {Id }}$ et est donc dans $\operatorname{Bir}\left(\mathcal{M}(2 ; \mathbb{C}) ; \Phi_{\text {Id }}\right)$ dès qu'il est inversible. Visiblement tous les $\sigma_{\mathrm{P}}$ et $\mathrm{M} \mapsto{ }^{\mathrm{t}} \mathrm{M}^{-1}$ ont cette propriété; par contre les involutions $\mathcal{T}$ et $\iota$ ne l'ont pas. Tous les éléments de $\operatorname{Bir}\left(\mathcal{M}(2 ; \mathbb{C}) ; \Phi_{\text {Id }}\right)$ dont la décomposition en les $\sigma_{\mathrm{P}}, \mathcal{T}$ et $\iota$ font apparaître autant de fois $\mathcal{T}$ et $\iota$ satisfont cette propriété.

Problème 2. Quelles sont les transformations rationnelles qui commutent à $\Phi_{\text {Id }}$ ?

\section{4. «PERTURBATIONS SPÉCIALES $\gg$ DES APPLICATIONS MONOMIALES COMPATIBLES À LA CONJUGAISON}

Nous considérons dans ce qui suit quelques transformations spéciales susceptibles de posséder un centralisateur suffisamment « gros » tout en présentant une dynamique plus « riche $\gg$ que celle de $\Phi_{\text {Id }}$.

Les applications monomiales de degré deux $\mathrm{M} \mapsto \mathrm{A}_{1} \mathrm{MA}_{2} \mathrm{MA}_{3}$ sont conjuguées à celles du type $\mathrm{AM}^{2} \mathrm{~B}$ (Remarque 1.3) ; nous allons nous concentrer sur le cas spécial $\mathrm{B}=\mathrm{Id}$. Soit $\mathrm{A}$ dans $\mathrm{GL}(2 ; \mathbb{C})$. Considérons l'application monomiale $\Phi_{\mathrm{A}}$ de $\mathcal{M}(2 ; \mathbb{C})$ dans lui-même définie par $\Phi_{\mathrm{A}}(\mathrm{M})=\mathrm{AM}^{2}$. Remarquons que

$$
\left(\sigma_{\mathrm{P}}^{-1} \Phi_{\mathrm{A}} \sigma_{\mathrm{P}}\right)(\mathrm{M})=\left(\mathrm{P}^{-1} \mathrm{AP}\right) \mathrm{M}^{2} .
$$

Nous pouvons donc prendre A sous forme de JoRDAn. Dans un premier paragraphe nous allons considérer le cas où A est diagonale, dans le suivant nous traiterons l'autre éventualité.

4.1. Cas diagonalisable. Écrivons $\mathrm{A}$ sous la forme $\operatorname{diag}\left(\lambda_{1}, \lambda_{2}\right)$; remarquons que si $\lambda_{1}=\lambda_{2}$, alors $\Phi_{\mathrm{A}}$ est conjuguée à $\Phi_{\mathrm{Id}}$ par une homothétie. Dans la suite nous supposerons donc que $\lambda_{1}$ et $\lambda_{2}$ sont distincts. Quitte à conjuguer $\Phi_{\mathrm{A}}$, toujours par une homothétie, nous pouvons supposer que $\operatorname{det} \mathrm{A}=1$, i.e. $\lambda_{1} \lambda_{2}=1$. Posons $\lambda=\lambda_{1}$ et $\lambda_{2}=\frac{1}{\lambda}$; la condition $\lambda_{1} \neq \lambda_{2}$ implique $\lambda^{2} \neq 1$ ce que nous supposerons dans la suite. La quadrique de dimension 2 formée des matrices nilpotentes est envoyée sur $\mathbf{0}$ par $\Phi_{\mathrm{A}}$ et l'algèbre $\mathfrak{s l}(2 ; \mathbb{C})$ est encore contractée, cette fois sur $\{\mu \mathrm{A} \mid \mu \in \mathbb{C}\}$ qui est contenu dans $\mathcal{D}$. 
La restriction de $\Phi_{\mathrm{A}}$ à $\mathcal{D}$ est conjuguée à $\Phi_{\mathrm{Id}_{\mid \mathcal{D}}}$, ce que l'on voit sur l'expression de $\Phi_{\mathrm{A}}$ :

$$
\left[\begin{array}{ll}
x & y \\
z & t
\end{array}\right] \rightarrow\left[\begin{array}{cc}
\lambda\left(x^{2}+y z\right) & \lambda y(x+t) \\
\frac{1}{\lambda} z(x+t) & \frac{1}{\lambda}\left(t^{2}+y z\right)
\end{array}\right]
$$

Bien que $\Phi_{\mathrm{A}_{\mid \mathcal{D}}}$ soit conjuguée à $\Phi_{\operatorname{Id}_{\mid \mathcal{D}}}$ nous allons voir que $\Phi_{\mathrm{A}}$ n'est pas holomorphiquement conjuguée à $\Phi_{\mathrm{Id}}$. En effet, les points fixes de $\Phi_{\mathrm{A}}$ sont puisque $\lambda^{2} \neq 1$,

$$
\text { 0, } \quad \operatorname{diag}\left(\frac{1}{\lambda}, \lambda\right), \quad\left\{\left[\begin{array}{cc}
0 & 0 \\
z & \lambda
\end{array}\right] \mid z \in \mathbb{C}\right\}, \quad\left\{\left[\begin{array}{cc}
\frac{1}{\lambda} & y \\
0 & 0
\end{array}\right] \mid y \in \mathbb{C}\right\}
$$

alors que $\Phi_{\text {Id }}$ admet un ensemble de points fixes de dimension 2 : ceci est une obstruction à la conjugaison entre $\Phi_{\text {Id }}$ et $\Phi_{\mathrm{A}}$.

4.1.1. Fibrations et fonctions invariantes. Comme pour $\Phi_{\mathrm{Id}}$ la fibration $H:\left[\begin{array}{ll}x & y \\ z & t\end{array}\right] \rightarrow \frac{y}{z}$ est invariante. Néanmoins si $H \circ \Phi_{\mathrm{Id}}=H$, nous avons $H \circ \Phi_{\mathrm{A}}=$ $\lambda^{2} H$ : il y a donc seulement deux fibres fixes, celles correspondant aux matrices triangulaires supérieures, resp. inférieures. Le feuilletage associé à $y \frac{\partial}{\partial y}-z \frac{\partial}{\partial z}$ est encore invariant par $\Phi_{\mathrm{A}}$; en fait $\Phi_{\mathrm{A}}$ commute à toutes les transformations $\left(x, \mathrm{e}^{s} y, \mathrm{e}^{-s} z, t\right)$, $s \in \mathbb{C}$. En particulier l'ensemble des points périodiques, les ensembles invariants maximaux (resp. minimaux) sont eux-mêmes invariants par le flot $y \frac{\partial}{\partial y}-z \frac{\partial}{\partial z}$. Il en résulte que la seule possibilité pour que les points périodiques (de même période) soient isolés est qu'ils soient confinés dans $\mathcal{D}$. Notons que la fibration det $=$ cte est invariante.

Comme nous l'avons fait pour $\Phi_{\text {Id }}$ nous nous intéressons au corps des fonctions invariantes $\mathrm{K}\left(\Phi_{\mathrm{A}}\right)$ pour $\mathrm{A}=\operatorname{diag}\left(\frac{1}{\lambda}, \lambda\right)$ générique. Pour celà nous étudions $\Phi_{\mathrm{A}}$ au voisinage du point fixe diag $\left(\frac{1}{\lambda}, \lambda\right)$. On vérifie que la matrice jacobienne de $\Phi_{\mathrm{A}}$ en ce point fixe est

$$
\left[\begin{array}{cccc}
2 & 0 & 0 & 0 \\
0 & \lambda\left(\lambda+\frac{1}{\lambda}\right) & 0 & 0 \\
0 & 0 & \frac{1}{\lambda}\left(\lambda+\frac{1}{\lambda}\right) & 0 \\
0 & 0 & 0 & 2
\end{array}\right]
$$

Ainsi, pour $\lambda$ non résonant (on demande que $2^{p} \lambda^{q}\left(\lambda+\frac{1}{\lambda}\right)^{r}=1,(p, q, r) \in \mathbb{Z}$ ait une seule solution, la solution triviale $(p, q, r)=(0,0,0))$, le germe de $\Phi_{\mathrm{A}}$ en diag $\left(\lambda, \frac{1}{\lambda}\right)$ est formellement linéarisable. Nous en déduisons qu'à conjugaison formelle près les fonctions méromorphes (formelles) invariantes par le germe de $\Phi_{\mathrm{A}}$ en diag $\left(\frac{1}{\lambda}, \lambda\right)$ sont les fonctions du type $h(x / t), h$ rationnelle. Soit maintenant $f$ dans $\mathrm{K}\left(\Phi_{\mathrm{A}}\right)$ non constante. Il n'est pas difficile de voir, en vertu de ce qui précède, que $f$ est non constante sur le 2-plan $y=z=0$. Mais dans ce 2 -plan la restriction de $\Phi_{\mathrm{A}}$ est donnée par $(x, t) \mapsto\left(\lambda x^{2}, \frac{1}{\lambda} t^{2}\right)$ qui est conjuguée à $\left(x^{2}, t^{2}\right)$. Or les fonctions invariantes par $\left(x^{2}, t^{2}\right)$ sont constantes. Il s'en suit la :

Proposition 4.1. Pour $\lambda$ non résonant $\mathrm{K}\left(\Phi_{\mathrm{A}}\right)=\mathbb{C}$.

Remarque 4.2. Pour certaines valeurs spéciales de $\lambda$ le corps $\mathrm{K}\left(\Phi_{\mathrm{A}}\right)$ ne se réduit pas aux constantes. En effet comme on l'a vu $H \circ \Phi_{\mathrm{A}}=\lambda^{2} H$; en particulier si $\lambda$ est une racine $2 k$-ième de l'unité, alors la fonction $(y / z)^{k}$ est invariante par $\Phi_{\mathrm{A}}$.

Problème 3. Décrire le corps $K\left(\Phi_{\mathrm{A}}\right)$ pour toutes les valeurs de $\lambda$. 
4.1.2. Étude des points périodiques pour $\lambda$ générique. Suivant la nature de $\lambda$ le comportement des points périodiques s'avère différent, et différent aussi du cas $\Phi_{\text {Id }}$ étudié précédemment. Supposons que $\lambda$ ne soit pas racine de l'unité. Puisque $H \circ \Phi_{\mathrm{A}}=\lambda^{2} H$ les points périodiques de $\Phi_{\mathrm{A}}$ sont contenus dans les hyperplans $z=0$ et $y=0$. La restriction $\Phi_{\mathrm{A}_{\mid z=0}}$ est conjuguée à $\Psi(x, y, t)=\left(x^{2}, y\left(x+\lambda^{2} t\right), t^{2}\right)$; on constate que

$$
\Psi^{n}(x, y, t)=\left(x^{2^{n}}, y \prod_{i=0}^{n-1}\left(x^{2^{i}}+\lambda^{2} t^{2^{i}}\right), t^{2^{n}}\right) .
$$

Notons que si $(\underline{x}, \underline{y}, \underline{t})$ est périodique pour $\Psi$, alors $(\underline{x}, \underline{t})$ l'est pour $\chi_{2}$; il y a donc quatre possibilités pour $(\underline{x}, \underline{t})$ :

(a) $(\underline{x}, \underline{t})=(0,0)$;

(b) $\underline{x}$ et $\underline{t}$ sont des racines $\left(2^{n}-1\right)$-ième de l'unité;

(c) $\underline{x}=0$ et $\underline{t}$ est une racine $\left(2^{n}-1\right)$-ième de l'unité;

(d) $\underline{t}=0$ et $\underline{x}$ est une racine $\left(2^{n}-1\right)$-ième de l'unité.

Examinons ces possibilités au cas par cas.

(a) Notons que si $(\underline{x}, \underline{t})=(0,0)$, alors $\underline{y}=0$.

(b) Considérons l'ensemble dénombrable $\Lambda_{n}$ défini par

$$
\Lambda_{n}=\left\{(x, t, \lambda) \in \mathbb{C}^{3} \mid x^{2^{n}-1}=1, t^{2^{n}-1}=1, \lambda \prod_{i=0}^{n-1}\left(x^{2^{i}}+\lambda^{2} t^{2^{i}}\right)=1\right\}
$$

et l'ensemble $\Lambda=\operatorname{pr}_{3}\left(\bigcup_{n} \Lambda_{n}\right)$ où $\operatorname{pr}_{3}$ désigne la troisième projection.

Un argument de BAIRE assure que pour $\lambda$ générique on a $\lambda^{n-1} \prod_{i=0}^{n-1}\left(\underline{x}^{2^{i}}+\lambda^{2} \underline{t}^{2^{i}}\right) \neq$ 1 pour tout choix $\underline{x}$ et $\underline{t}$ de racines $\left(2^{n}-1\right)$-ième de l'unité. Par suite, pour $\lambda$ n'appartenant pas à $\Lambda$, les points périodiques $(\underline{x}, \underline{y}, \underline{t})$ de $\Psi$ tels que $\underline{x t} \neq 0$ sont exactement l'ensemble

$$
\bigcup_{n \geq 0}\left\{(\xi, 0, \eta) \mid \xi^{2^{n}-1}=\eta^{2^{n}-1}=1\right\} .
$$

Lorsque $(\underline{x}, \underline{t}, \lambda)$ appartient à $\Lambda_{n}$ (en particulier $\lambda$ appartient à $\left.\Lambda\right),(\underline{x}, y, \underline{t})$ est périodique pour $\Psi$, pour tout $y$. Par exemple $\left(\mathrm{j}, \mathrm{j}^{2}, 2\right)$ appartient à $\Lambda_{2}$ et $\left(\mathrm{j}, y, 0, \mathrm{j}^{2}\right)$ est un point périodique de période 2 de l'application correspondante.

(c) Passons maintenant au cas où par exemple $\underline{t}=0$ et $\underline{x}^{2^{n}-1}=1$. On vérifie que $\Psi^{n}(\underline{x}, y, 0)=(\underline{x}, y, 0)$.

(d) Lorsque $\underline{x}=0$ et $\underline{t}^{2^{n}-1}=1$ on constate que $\Psi^{n}(0, y, \underline{t})=\left(0, \lambda^{2} y, \underline{t}\right)$.

Bien sûr tout ce qui est dit pour la restriction de $\Phi_{\mathrm{A}}$ à $z=0$ peut être répété pour la restriction à $y=0$. Nous en déduisons la :

Proposition 4.3. Pour $\lambda$ générique (i.e. $\lambda$ n'appartient pas à $\Lambda$ ) l'adhérence des points périodiques de $\Phi_{\mathrm{A}}$ est constituée

- d'un tore $\mathbb{S}^{1} \times \mathbb{S}^{1}$ contenu dans $y=z=0$

- de deux $\mathbb{S}^{1} \times \mathbb{C}$, précisément $\frac{1}{\lambda} \mathbb{S}^{1} \times \mathbb{C} \times\{0\} \times\{0\}$ et $\{0\} \times\{0\} \times \mathbb{C} \times \lambda \mathbb{S}^{1}($ en identifiant $\left.\mathcal{M}(2 ; \mathbb{C})=\left\{\left[\begin{array}{cc}x & y \\ z & t\end{array}\right]\right\} \grave{a} \mathbb{C}^{4}=\{(x, y, z, t) \mid x, y, z, t \in \mathbb{C}\}\right) ;$

- de la matrice nulle $\mathbf{0 .}$

En particulier lorsque $\lambda$ n'est pas dans $\Lambda$ les points périodiques sont d'adhérence de ZARISKI $\{y=z=0\} \cup\{z=t=0\} \cup\{x=y=0\}$ alors que les points 
périodiques de $\Phi_{\text {Id }}$ sont ZARISKI denses. La description des points périodiques de $\Phi_{\mathrm{A}}$ dans le cas non générique (par exemple $\lambda$ racine de l'unité) semble délicate.

Remarque 4.4. La dynamique de $\Phi_{\mathrm{A}}: \mathcal{M}(2 ; \mathbb{C}) \rightarrow \mathcal{M}(2 ; \mathbb{C})$ peut être précisée par l'étude de ses points fixes ou plus généralement périodiques à l'infini. Pour celà considérons l'application $\widetilde{\Phi_{\mathrm{A}}}: \mathbb{P}^{4}(\mathbb{C}) \rightarrow \mathbb{P}^{4}(\mathbb{C})$ définie par

$$
(x: y: z: t: s) \mapsto\left(\lambda\left(x^{2}+y z\right): \lambda y(x+t): \frac{z(x+t)}{\lambda}: \frac{t^{2}+y z}{\lambda}: s^{2}\right) .
$$

On vérifie que $\widetilde{\Phi_{\mathrm{A}}}$ s'exprime de la façon suivante dans la carte $x=1$,

$$
\left(\frac{y(1+t)}{1+y z}, \frac{z(1+t)}{\lambda^{2}(1+y z)}, \frac{t^{2}+y z}{\lambda^{2}(1+y z)}, \frac{s^{2}}{\lambda(1+y z)}\right) .
$$

Dans l'hyperplan $y=0$ qui est invariant, le point $(z=0, t=0, s=0)$ est un attracteur si $|\lambda|>1$. Dans la carte $t=1$, où $\widetilde{\Phi_{\mathrm{A}}}$ s'exprime cette fois sous la forme

$$
\left(\frac{\lambda^{2}\left(x^{2}+y z\right)}{1+y z}, \frac{\lambda^{2} y(1+x)}{1+y z}, \frac{z(1+x)}{1+y z}, \frac{\lambda s^{2}}{1+y z}\right),
$$

on voit que $z=0$ est invariant et dans ce 3-plan le point $(x=0, y=0, s=0)$ est un attracteur si $|\lambda|<1$. Ainsi si $|\lambda| \neq 1$ on trouve un hyperplan invariant contenant un point fixe attractant dans cet hyperplan. De même on peut préciser la nature des points périodiques à l'infini, tout du moins dans ces hyperplans invariants.

Problème 4. Décrire les points périodiques des applications $\Phi_{\mathrm{A}}$ pour toute valeur de $\lambda$.

4.1.3. Étude de quelques orbites non périodiques. Nous allons maintenant préciser la nature de quelques orbites non périodiques. Dans toute étude de systèmes dynamiques on s'intéresse à des problèmes de stabilité, de «non-explosion », ce qui conduit à caractériser autant que faire se peut les orbites bornées. L'idéal serait d'en avoir une description complète pour chaque $\Phi_{\mathrm{A}}$. Nous allons simplement en donner quelques constructions. Puisque nous pouvons nous ramener à $\lambda_{1} \lambda_{2}=1$ nous avons $\operatorname{det} \Phi_{\mathrm{A}}(\mathrm{M})=(\operatorname{det} \mathrm{M})^{2}$ et par suite $\operatorname{det} \Phi_{\mathrm{A}}^{k}(\mathrm{M})=(\operatorname{det} \mathrm{M})^{2^{k}}$. Il en résulte que pour $\mathrm{M}$ tel que $|\operatorname{det} \mathrm{M}|>1$, la suite $\left(\Phi_{\mathrm{A}}^{k}(\mathrm{M})\right)_{k}$ tend vers l'infini. Pour A fixé considérons le bassin d'attraction $\mathrm{W}_{\mathrm{A}}^{s}(\mathbf{0})$ de la matrice nulle; c'est un domaine disqué, i.e. si $\mathrm{M}$ appartient à $\mathrm{W}_{\mathrm{A}}^{s}(\mathbf{0})$, alors le disque $\{\mu \mathrm{M}|| \mu \mid \leq 1\}$ est lui aussi dans $\mathrm{W}_{\mathrm{A}}^{s}(\mathbf{0})$. Le fait que $\Phi_{\mathrm{A}}$ commute aux applications linéaires $f_{s}(x, y, z, t)=\left(x, \mathrm{e}^{s} y, \mathrm{e}^{-s} z, t\right)$ implique que $\mathrm{W}_{\mathrm{A}}^{s}(\mathbf{0})$ est non borné. Désignons par $\|$.$\| la norme \sup \operatorname{sur} \mathcal{M}(2 ; \mathbb{C})$. Soient $K=\sup \left(\lambda, \frac{1}{\lambda}\right)$ et $\mathrm{M}$ dans le polydisque $\Delta(\rho)$ de rayon $\rho$; nous avons l'inégalité $\left\|\Phi_{\mathrm{A}}^{k}(\mathrm{M})\right\| \leq 2 K \rho^{2}$ et par conséquent $\left\|\Phi_{\mathrm{A}}^{k}(\mathrm{M})\right\| \leq(2 K)^{2^{k}-1} \rho^{2^{k}}$. Il en résulte l'inclusion du polydisque $\Delta\left(\frac{1}{2 K}\right)$ dans $\mathrm{W}_{\mathrm{A}}^{s}(\mathbf{0})$ ainsi que de son saturé $\bigcup_{s} f_{s}\left(\Delta\left(\frac{1}{2 K}\right)\right)$ par le flot $f_{s}$. Ceci donne d'ailleurs une preuve du fait que $\mathrm{W}_{\mathrm{A}}^{s}(\mathbf{0})$ est ouvert. Il est assez simple de produire des orbites bornées dans les plans invariants $y=0$ et $z=0$. Plaçons-nous par exemple dans l'hyperplan invariant $z=0$; nous travaillons de nouveau avec $\Psi$ dont l'itéré $n$-ième s'écrit pour $x$ non nul

$$
\Psi^{n}(x, y, t)=\left(x^{2^{n}}, y x^{2^{n}-1} \prod_{i=0}^{n-1}\left(1+\lambda^{2}\left(\frac{t}{x}\right)^{2^{i}}\right), t^{2^{n}}\right) .
$$

En particulier les orbites des points $(x, y, 0)$ sont faciles à décrire et produisent des orbites bornées lorsque $|x|=1$ (ou 0 ). Remarquons que si $x$ et $t$ sont fixés tels 
que $|t|<|x|$, alors le produit infini $\prod_{i=0}^{\infty}\left(1+\lambda^{2}\left(\frac{t}{x}\right)^{2^{i}}\right)$ converge vers un nombre $\mu=\mu\left(\frac{t}{x}\right)$. Considérons l'application $\Psi_{0}$ définie par $\Psi_{0}(x, y, t)=\left(x^{2}, x y, t^{2}\right)$ (i.e. qui correspond au cas $\lambda=0)$. Nous avons

$$
\left\|\Psi^{n}(x, y, t)-\Psi_{0}^{n}(x, y, t)\right\|=|y| \cdot\left|x^{2^{n}-1}\right| \cdot\left|\prod_{i=0}^{n-1}\left(1+\lambda^{2}\left(\frac{t}{x}\right)^{2^{i}}\right)-1\right| .
$$

En particulier pour $|t|<|x|<1$, nous avons $\lim _{n \rightarrow+\infty} \Psi^{n}(x, y, t)=0$ indépendamment de $y$. Ainsi l'ensemble $\{(x, y, t)|| t|<| x \mid<1\}$ est contenu dans le bassin d'attraction de $\mathbf{0}$ pour l'application $\Psi$ ce qui donne un renseignement supplémentaire pour $\mathrm{W}_{\mathrm{A}}^{s}(\mathbf{0})$. Lorsque $|\underline{x}|=1,|\underline{t}|<1$ et $y$ quelconque $\mathcal{O}\left((\underline{x}, \underline{y}, \underline{t}) ; \Psi_{0}\right)$ est contenue dans $|\underline{x}|=1,|y|=|\underline{y}|$ alors que $\lim _{n \rightarrow+\infty} \underline{t}^{2^{n}}=0$. Dans cette situation on constate que $\left\|\Psi^{n}(\underline{x}, \underline{y}, \underline{t})-\Psi_{0}^{n}(\underline{x}, \underline{y}, \underline{t})\right\|$ est bornée et donc que $\mathcal{O}((\underline{x}, \underline{y}, \underline{t}) ; \Psi)$ est bornée.

Remarquons que le plan $x=t$ (qui est dans le bord du domaine $|t|<|x|$ ) est invariant par $\Psi$. La restriction de $\Psi$ à ce plan s'écrit $\Psi(x, y)=\left(x^{2},\left(1+\lambda^{2}\right) x y\right)$; elle est conjuguée via l'application birationnelle $(x, x y)$ à $\varphi=\left(x^{2},\left(1+\lambda^{2}\right) y\right)$ dont l'itéré nième est $\left(x^{2^{n}},\left(1+\lambda^{2}\right)^{n} y\right)$. Pour tout $x$ non nul, la nature des orbites de $\Psi_{\mid x=t}$ peut se déduire de celles de $\varphi$; plus précisément $\varphi$ contracte $x=t=0$ (l'axe des $y$ ) sur la matrice nulle. En dehors de $x=0,\left(x^{2},\left(1+\lambda^{2}\right) x y\right)$ est holomorphiquement conjugué à $\left(x^{2},\left(1+\lambda^{2}\right) y\right)$. Par exemple lorsque $\underline{x}$ est de module 1 générique et $1+\lambda^{2}$ est aussi de module 1 générique, les orbites de $(\underline{x}, y)$ sont d'adhérence des tores réels de dimension 2 .

Une façon plus précise d'appréhender l'étude de l'application $\Psi \sim \Phi_{\mathrm{A} \mid z=0}$ est de la conjuguer par une transformation birationnelle bien choisie. Par exemple si $E: \mathbb{C}^{3} \rightarrow \mathbb{C}^{3}$ est l'application d'éclatement de l'origine définie par $E(x, u, v)=$ $(x, x u, x v)$ nous avons le diagramme commutatif

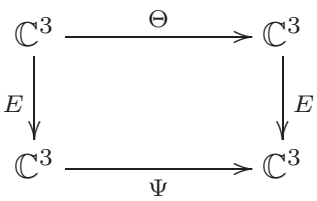

avec $\Theta(x, u, v)=\left(x^{2}, u\left(1+\lambda^{2} v\right), v^{2}\right)$. L'itéré $n$-ième de $\Theta$ est donné par

$$
\Theta^{n}(x, u, v)=\left(x^{2^{n}}, u T_{n}^{\lambda}(v), v^{2^{n}}\right), \quad T_{n}^{\lambda}(v)=\prod_{i=0}^{n-1}\left(1+\lambda^{2} v^{2^{i}}\right) .
$$

Contrôler les $v$ appartenant à $\mathbb{S}^{1}$ pour lesquels les produits $\prod_{i=0}^{n-1}\left(1+\lambda^{2} v^{2^{i}}\right)$ restent bornés permet d'exhiber des orbites bornées de $\Theta$ et une orbite bornée de $\Theta$ induit une orbite bornée de $\Psi$. En particulier chaque fois que l'on sait borner l'ensemble $\left\{T_{n}^{\lambda}(v) \mid n \in \mathbb{N}\right\}$ pour certaines valeurs de $\lambda$ et $v$ on sait construire une orbite bornée de $\Psi$. Voici un exemple : supposons que $\lambda$ soit un réel, $0<\lambda<1$, et 
que $v$ soit une racine cubique de l'unité, par exemple $\mathrm{j}$. Nous avons

$$
\begin{aligned}
& T_{1}^{\lambda}(\mathrm{j})=\left(1+\lambda^{2} \mathrm{j}\right), \\
& T_{2}^{\lambda}(\mathrm{j})=\left(1+\lambda^{2} \mathrm{j}\right)\left(1+\lambda^{2} \mathrm{j}^{2}\right), \\
& T_{3}^{\lambda}(\mathrm{j})=\left(1+\lambda^{2} \mathrm{j}\right)^{2}\left(1+\lambda^{2} \mathrm{j}^{2}\right), \\
& T_{4}^{\lambda}(\mathrm{j})=\left(1+\lambda^{2} \mathrm{j}\right)^{2}\left(1+\lambda^{2} \mathrm{j}^{2}\right)^{2}, \ldots
\end{aligned}
$$

Une induction élémentaire montre que pour tout $n \geq 0$ les $\left|T_{n}^{\lambda}(\mathrm{j})\right|$ sont strictement plus petits que 1 . Par suite si $|x| \leq 1$, alors $\mathcal{O}\left((x, y, \mathrm{j}) ; \Phi_{\mathrm{A}}\right)$ est bornée (toujours sous la condition $0<\lambda<1$ ).

Problème 5. Pour $\lambda$ fixé donner les $v$ de module inférieur ou égal à 1 pour lesquels $\left\{T_{n}^{\lambda}(v) \mid n \in \mathbb{N}\right\}$ est borné.

Soit $\mathrm{M}$ dans $\mathcal{M}(2 ; \mathbb{C})$; supposons que $\mathcal{O}\left(\mathrm{M} ; \Phi_{\mathrm{A}}\right)$ soit bornée. Alors les points limites de $\mathcal{O}\left(\mathrm{M} ; \Phi_{\mathrm{A}}\right)$ sont encore à orbites bornées. Si $|\operatorname{det} \mathrm{M}|=1$, alors $\overline{\mathcal{O}\left(\mathrm{M} ; \Phi_{\mathrm{A}}\right)}$ est contenu dans $\{\mathrm{M} \in \mathcal{M}(2 ; \mathbb{C}) \mid \operatorname{det} \mathrm{M}=0\}$, tandis que si $|\operatorname{det} \mathrm{M}|<1$ ces points limites sont dans l'hypersurface $\{\mathrm{M} \in \mathcal{M}(2 ; \mathbb{C}) \mid \operatorname{det} \mathrm{M}=0\}$. Il est donc naturel de rechercher les orbites bornées dans les deux ensembles

$$
\{\mathrm{M} \in \mathcal{M}(2 ; \mathbb{C}) \mid \operatorname{det} \mathrm{M}=0\} \quad \text { et } \quad\{\mathrm{M} \in \mathcal{M}(2 ; \mathbb{C})|| \operatorname{det} \mathrm{M} \mid=1\}
$$

ce que nous aborderons dans ce qui suit.

4.1.4. Orbites bornées dans $\{\mathrm{M} \in \mathcal{M}(2 ; \mathbb{C}) \mid \operatorname{det} \mathrm{M}=0\}$. Soit $\mathrm{M}=\left[\begin{array}{ll}x & y \\ z & t\end{array}\right]$ dans $\mathcal{M}(2 ; \mathbb{C})$ de déterminant nul ; on constate que $\Phi_{\mathrm{A}}(\mathrm{M})=(x+t)\left(\lambda x, \lambda y, \frac{z}{\lambda}, \frac{t}{\lambda}\right)$. L'application $\mathbb{P}\left(\Phi_{\mathrm{A}}\right): \mathbb{P}^{3}(\mathbb{C}) \rightarrow \mathbb{P}^{3}(\mathbb{C})$ coïncide en restriction à la quadrique $\{\mathrm{M} \in$ $\mathcal{M}(2 ; \mathbb{C}) \mid \operatorname{det} \mathrm{M}=0\}$ avec $(x: y: z: t) \mapsto(\lambda x: \lambda y: z / \lambda: t / \lambda)$ qui est linéaire. Plus généralement nous avons

$$
\Phi_{\mathrm{A}}^{n}(\mathrm{M})=P_{n} \cdot\left(\lambda^{n} x, \lambda^{n} y, \frac{z}{\lambda^{n}}, \frac{t}{\lambda^{n}}\right), \quad P_{n}=\prod_{i=0}^{n-1}\left(\lambda^{i} x+\frac{t}{\lambda^{i}}\right)^{2^{n-i-1}} .
$$

En particulier on constate le phénomène de résonance suivant : si $\mathrm{M}=\left[\begin{array}{ll}x & y \\ z & t\end{array}\right]$ dans $\mathcal{M}(2 ; \mathbb{C})$ satisfait $x t-y z=0$ et $\lambda^{k} x+\frac{t}{\lambda^{k}}=0$ pour un certain $k$, alors $\Phi_{\mathrm{A}}^{n}(\mathrm{M})=\mathbf{0}$ pour $n \geq k$. Nous obtenons donc, tout du moins pour $\lambda$ générique (précisément pour $\lambda$ non racine de l'unité), une infinité de surfaces quadratiques

$$
\mathcal{Q}_{k}(\mathrm{~A})=\left\{\left[\begin{array}{cc}
x & y \\
z & t
\end{array}\right] \in \mathcal{M}(2 ; \mathbb{C}) \mid \operatorname{det} \mathrm{M}=0, \lambda^{k} x+t / \lambda^{k}=0\right\}
$$

qui sont envoyées sur $\mathbf{0}$ après un nombre fini d'itérations. Comme le bassin d'attraction de $\mathbf{0}$ est ouvert, il existe des voisinages ouverts de ces surfaces quadratiques contenus dans $\mathrm{W}_{\mathrm{A}}^{s}(\mathbf{0})$, ce qui produit évidemment des orbites bornées. Rappelons que dans $\{\mathrm{M} \in \mathcal{M}(2 ; \mathbb{C}) \mid \operatorname{det} \mathrm{M}=0\}$ il y a des orbites non bornées (celles de $(x, y, 0,0)$ avec $|x|$ grand) et des orbites bornées non contenues dans $\mathrm{W}_{\mathrm{A}}^{s}(\mathbf{0})$, par exemple celles des points $(x, y, 0,0)$ avec $|x|=1 / \lambda$ qui sont toutefois dans le bord de $\mathrm{W}_{\mathrm{A}}^{s}(\mathbf{0})$.

Sur http ://math.cmaisonneuve.qc.ca/alevesque/chaos_fract/Julia/Julia.html on trouve un programme permettant de tracer l'ensemble de JULIA d'une application holomorphe de $\mathbb{P}^{1}(\mathbb{C})$ dans lui-même. Nous proposons une « adaptation » 
de ce programme à certaines transformations polynomiales réelles. Plus précisément notons $\mathbb{D}(0, r) \subset \mathbb{R}^{2}$ le disque de rayon $r$ centré en 0 et $\Delta(\rho)$ le polydisque de rayon $\rho:$

$$
\Delta(\rho)=\left\{(y, t) \in \mathbb{R}^{2}|| y|<\rho,| t \mid<\rho\right\} .
$$

Soit $f$ une transformation polynomiale du plan $\mathbb{R}^{2}$. Soient $m=\left(x_{0}, x_{1}\right)$ un point de $\Delta(\rho)$ et $\kappa$ un entier strictement positif. On appelle temps de sortie $N(m ; r, \rho, \kappa)$, relatif aux données de contrôle $r, \rho, \kappa$, du point $m$ de l'intersection $\Delta(\rho) \cap \mathbb{D}(0, r)$ le plus grand entier $n$ dans $[0, \ldots, \kappa]$ tel que

$$
f^{k}(m) \in \mathbb{D}(0, r) \quad \forall 0 \leq k \leq n .
$$

Considérons le spectre (continu) des couleurs [rouge... orange... jaune... vert... bleu....indigo...rouge] que l'on discrétise en $\kappa+1$ intervalles $\left[I_{0}, \ldots, I_{\kappa}\right]=$ [rouge... jaune...bleu... rouge]. Soit $(r, \rho, \kappa)$ un triplet de contrôle. Si $N(m ; r, \rho, \kappa)=k$, on colore le point $m$ de la couleur $\operatorname{Col}(m)=I_{k}$. Comme $I_{0}=I_{\kappa}=$ rouge, les points colorés en rouge sont ceux pour lesquels le temps de passage est 0 (sortie immédiate) ou $\kappa$ (pas de sortie au bout de $\kappa$ itérations); sur les figures le bord de l'ensemble $\mathrm{Col}^{-1}\left(\mathrm{I}_{0}\right)$ est approché par la couleur $I_{1} \sim$ orangé.

Nous allons appliquer cette procédure à $\left(\lambda x(x+t), \frac{t(x+t)}{\lambda}\right)$, application qui décrit la dynamique de la restriction de $\Phi_{\mathrm{A}}$ à $\{\mathrm{M} \in \mathcal{M}(2 ; \mathbb{R}) \mid \operatorname{det} \mathrm{M}=0\}$. Nous obtenons pour $\rho=10, r=30$ :

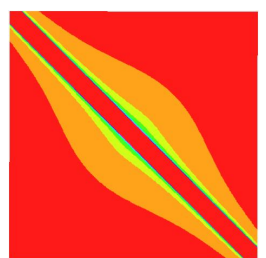

$\kappa=10, \lambda=1$

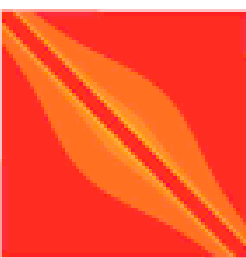

$\kappa=75, \lambda=1$

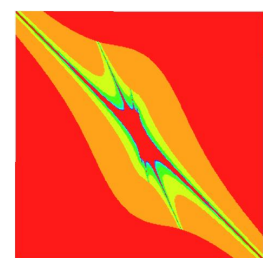

$\kappa=10, \lambda=1.5$

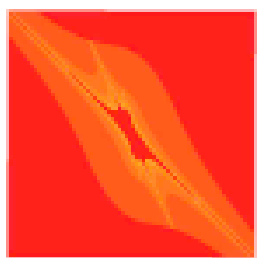

$\kappa=75, \lambda=1.5$

Les deux premières figures représentent une approximation de la projection sur le plan des $(x, t)$ du bassin d'attraction de $\mathbf{0}$ par $\Phi_{\mathrm{Id}}$ intersecté avec $\{\mathrm{M} \in$ $\mathcal{M}(2 ; \mathbb{R}) \mid \operatorname{det} \mathrm{M}=1\}$. On vérifie en effet que, dans ce cas, la bande $|x+t|<1$ est exactement le bassin d'attraction de l'origine pour l'application considérée. Le domaine « étoilé » des troisième et quatrième dessins représente comme précédemment la projection sur le plan des $(x, t)$ de $\mathrm{W}_{\mathrm{A}}^{s}(\mathbf{0})$ intersecté avec $\{\mathrm{M} \in$ $\mathcal{M}(2 ; \mathbb{R}) \mid \operatorname{det} \mathrm{M}=0\}$. On distingue ici quelques droites $\lambda^{k} x+\frac{t}{\lambda^{k}}=0$ et leur voisinage contenus dans le bassin d'attraction. Au vu de ces figures on peut penser que le bord du bassin d'attraction $\mathrm{W}_{\mathrm{A}}^{s}(\mathbf{0})$ n'est plus LEVI-plat.

4.1.5. Orbites bornées dans $\{\mathrm{M} \in \mathcal{M}(2 ; \mathbb{C})|| \operatorname{det} \mathrm{M} \mid=1\}$. La dynamique dans $\{\mathrm{M} \in \mathcal{M}(2 ; \mathbb{C})|| \operatorname{det} \mathrm{M} \mid=1\}$ semble difficile d'abord pour $\lambda$ quelconque. Nous nous contentons de quelques remarques concernant l'hypersurface invariante $\{\mathrm{M} \in$ $\mathcal{M}(2 ; \mathbb{C}) \mid \operatorname{det} \mathrm{M}=1\}$. La matrice $\mathrm{M}_{0}=\left[\begin{array}{cc}\frac{1}{\lambda} & 0 \\ 0 & \lambda\end{array}\right]$ est fixe pour la transformation $\Phi_{\mathrm{A}}$ et appartient à la quadrique $\{\mathrm{M} \in \mathcal{M}(2 ; \mathbb{C}) \mid \operatorname{det} \mathrm{M}=1\}$. La matrice 
jacobienne de $\Phi_{\mathrm{A}}$ en $\mathrm{M}_{0}$ est la suivante

$$
\operatorname{Jac}\left(\Phi_{\mathrm{A}}\right)_{\left(\mathrm{M}_{0}\right)}=\left[\begin{array}{cccc}
2 & 0 & 0 & 0 \\
0 & \lambda\left(\lambda+\frac{1}{\lambda}\right) & 0 & 0 \\
0 & 0 & \frac{1}{\lambda}\left(\lambda+\frac{1}{\lambda}\right) & 0 \\
0 & 0 & 0 & 2
\end{array}\right] ;
$$

on constate un phénomène de résonance entre les valeurs propres. Remarquons que si l'argument de $\lambda$ appartient à ] $-\pi / 4, \pi / 4\left[\right.$, alors les valeurs propres de $\operatorname{Jac}\left(\Phi_{\mathrm{A}}\right)_{\left(\mathrm{M}_{0}\right)}$ sont en module strictement supérieures à 1 . Sous cette hypothèse le point fixe $\mathrm{M}_{0}$ est un répulseur, i.e. il existe un voisinage $\mathcal{V}\left(\mathrm{M}_{0}\right)$ de $\mathrm{M}_{0}$ tel que $\mathcal{V}\left(\mathrm{M}_{0}\right) \subsetneq \Phi_{\mathrm{A}}\left(\mathcal{V}\left(\mathrm{M}_{0}\right)\right)$. Mais ce fait n'est pas universel puisque pour certaines valeurs de $\lambda$ on trouve des valeurs propres de module plus petit que 1 .

En appliquant la « procédure JULIA » introduite précédemment à

$$
\left(\lambda\left(x^{2}+x t-1\right), \frac{\left(t^{2}+x t-1\right)}{\lambda}\right)
$$

qui décrit la dynamique de la restriction de $\Phi_{\mathrm{A}}$ à $\{\mathrm{M} \in \mathcal{M}(2 ; \mathbb{R}) \mid \operatorname{det} \mathrm{M}=1\}$, nous obtenons par exemple les figures qui suivent pour $\rho=10, r=3$ et les paramètres $\lambda=1$ et 1.5 .

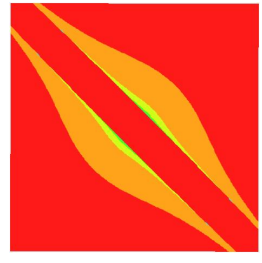

$\kappa=10, \lambda=1$

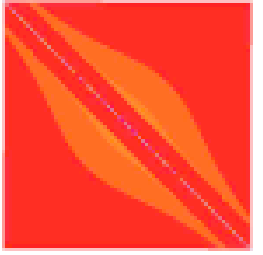

$\kappa=75, \lambda=1$

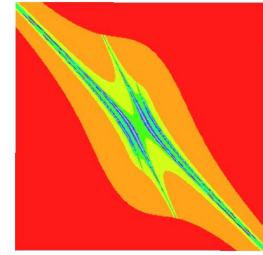

$\kappa=10, \lambda=1.5$

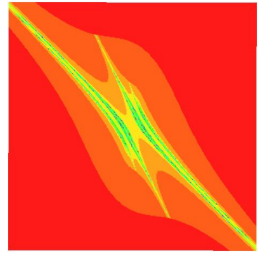

$\kappa=75, \lambda=1.5$

Notons qu'il y a une similitude certaine avec les figures précédentes.

4.1.6. Précisions sur la dynamique dans le cas quaternionique. Lorsque la matrice A est une matrice de quaternions nous pouvons préciser le discours précédent.

L'itération des transformations polynomiales du corps des quaternions a été abordée dans [7] où les auteurs proposent une adaptation de la théorie de FATOU JULIA.

Considérons une application $\Phi_{\mathrm{A}}: \mathrm{M} \mapsto \mathrm{AM}^{2}$ avec cette fois $\mathrm{A}=\left[\begin{array}{cc}a & b \\ -\bar{b} & \bar{a}\end{array}\right]$ quaternionique. Quitte à faire agir une homothétie réelle $\mathrm{M} \mapsto \rho \mathrm{M}$ sur $\Phi_{\mathrm{A}}$ nous pouvons supposer que $\operatorname{det} \mathrm{A}=1$, i.e. que $\mathrm{A}$ est un quaternion de module 1. Mieux en conjuguant par une transformation $\mathrm{M} \mapsto \mathrm{BMB}^{-1}$ où $\mathrm{B}$ est encore un quaternion ad-hoc nous pouvons supposer que $\mathrm{A}$ est diagonale, i.e. $\mathrm{A}=\operatorname{diag}\left(\mathrm{e}^{\mathrm{i} \vartheta}, \mathrm{e}^{-\mathrm{i} \vartheta}\right)$. On constate donc, modulo ces modifications, que le corps $\mathbb{H}$ des quaternions

$$
\mathbb{H}=\left\{\left[\begin{array}{cc}
x & y \\
-\bar{y} & \bar{x}
\end{array}\right] \mid(x, y) \in \mathbb{C}^{2}\right\}
$$

est complètement invariant par $\Phi_{\mathrm{A}}$; il en est de même pour l'ensemble $\mathbb{H}_{1}$ des quaternions de module 1

$$
\mathbb{H}_{1}=\{\mathrm{M} \in \mathbb{H} \mid \operatorname{det} \mathrm{M}=1\} .
$$


Notons que $\mathbb{H}_{<1}=\{\mathrm{M} \in \mathbb{H}|| \operatorname{det} \mathrm{M} \mid<1\}$ est contenu dans le bassin d'attraction $\mathrm{W}_{\mathrm{A}}^{s}(\mathbf{0})$ de l'origine. Étant donné que $\mathbb{H}_{1}$ est invariant, ceci produit des orbites bornées non contenues dans $\partial \mathrm{W}_{\mathrm{A}}^{s}(\mathbf{0})$ mais dans son bord. Puisque les $\sigma_{\mathrm{D}}: \mathrm{M} \mapsto$ $\mathrm{DMD}^{-1}$, avec $\mathrm{D}$ diagonale, commutent avec $\Phi_{\mathrm{A}}$ les ensembles $\sigma_{\mathrm{D}}(\mathbb{H}), \sigma_{\mathrm{D}}\left(\mathbb{H}_{1}\right)$ sont invariants. Ceci donne deux autres exemples d'ensembles invariants non bornés :

- l'ensemble $\mathcal{H}$ de dimension réelle 5 qui est l'union des $\sigma_{\mathrm{D}}(\mathbb{H})$ :

$\mathcal{H}=\left\{\left[\begin{array}{cc}x & \xi y \\ -\frac{\bar{y}}{\xi} & \bar{x}\end{array}\right] \mid(x, y) \in \mathbb{C}^{2}, \xi \in \mathbb{C}^{*}\right\}=\left\{\left[\begin{array}{cc}x & y \\ \alpha \bar{y} & \bar{x}\end{array}\right] \mid(x, y) \in \mathbb{C}^{2}, \alpha \in \mathbb{R}_{<0}\right\} ;$

- ainsi que l'ensemble $\mathcal{H}_{1}$ de dimension réelle 4 qui est l'union des $\sigma_{\mathrm{D}}\left(\mathbb{H}_{1}\right)$ :

$$
\mathcal{H}_{1}=\left\{\left[\begin{array}{cc}
x & \xi y \\
-\frac{\bar{y}}{\xi} & \bar{x}
\end{array}\right] \mid(x, y) \in \mathbb{C}^{2}, \xi \in \mathbb{C}^{*}\right\} .
$$

Chaque élément de $\mathcal{H}_{1}$ a son orbite bornée contenue dans le bord du bassin d'attraction $\mathrm{W}_{\mathrm{A}}^{s}(\mathbf{0})$. Les matrices de la forme $\operatorname{diag}\left(\mathrm{e}^{\mathrm{i} \varphi}, \mathrm{e}^{\mathrm{i} \vartheta}\right)$ ont aussi une orbite bornée par $\Phi_{\mathrm{A}}$ et pour la plupart ne sont pas dans $\mathcal{H}_{1}$.

Nous avons réalisé quelques expériences numériques concernant la restriction de $\Phi_{\mathrm{A}}$ à $\mathbb{H}_{1}$. Avant de les présenter faisons quelques remarques élémentaires. L'étude de la restriction de $\Phi_{\mathrm{A}}$ à $\mathbb{H}$ se ramène bien sûr à celle de l'application analytique réelle de $\mathbb{C}^{2} \simeq \mathbb{R}^{4}$ dans lui-même (on garde la même notation) induite par les deux premières composantes :

$$
\Phi_{\mathrm{A}}:(x, y) \mapsto\left(\mathrm{e}^{\mathrm{i} \vartheta}\left(x^{2}-|y|^{2}\right), \mathrm{e}^{\mathrm{i} \vartheta} y(x+\bar{x})\right) .
$$

Sur $\mathbb{H}_{1}$ nous avons $|x|^{2}+|y|^{2}=1$ de sorte que $\Phi_{\mathrm{A}_{\mathbb{H}_{1}}}$ est fibrée

$$
\Phi_{\mathrm{A}_{\mathbb{H}_{1}}}:(x, y) \mapsto\left(\mathrm{e}^{\mathrm{i} \vartheta}\left(x^{2}+|x|^{2}-1\right), \mathrm{e}^{\mathrm{i} \vartheta} y(x+\bar{x})\right)=\left(\varphi_{\vartheta}(x), \mathrm{e}^{\mathrm{i} \vartheta} y(x+\bar{x})\right),
$$

i.e. la première composante ne dépend que de $x$.

Notons que la famille des cercles verticaux paramétrée par $\eta \mapsto\left(x, y \mathrm{e}^{\mathrm{i} \eta}\right)$ est globalement invariante. La seconde composante de $\Phi_{\mathrm{A}_{\mathbb{H}_{1}}}^{k}$ a pour argument $\varphi+k \vartheta$ avec $y=\rho \mathrm{e}^{\mathrm{i} \varphi}$ qui est aussi l'argument de l'itéré $k$-ième de $y$ suivant la rotation d'angle $\vartheta$. La première composante indique comment $\Phi_{\mathrm{A}}$ fait passer d'un cercle à l'autre. Par exemple si $\underline{x}$ est un point périodique de période $k$ de $\varphi_{\vartheta}$ alors l'application $\varphi_{\vartheta}^{k}$ est une rotation d'angle $k \vartheta$ sur le cercle $\mathcal{C}=\left\{(\underline{x}, y) \in \mathbb{H}_{1}\right\}$ de $\mathbb{H}_{1} \simeq \mathbb{S}^{3}$. Nous allons nous intéresser à cette première composante $\varphi_{\vartheta}$ que l'on identifie via $x=x_{1}+\mathrm{i} x_{2}$ à l'application toujours notée $\varphi_{\vartheta}$ :

$$
\varphi_{\vartheta}:\left(x_{1}, x_{2}\right) \mapsto\left(\cos \vartheta\left(2 x_{1}^{2}-1\right)-2 \sin \vartheta x_{1} x_{2}, 2 \cos \vartheta x_{1} x_{2}+\sin \vartheta\left(2 x_{1}^{2}-1\right)\right) .
$$

Les applications $\varphi_{\vartheta}$ respectent toutes le disque unité $\mathbb{D}(0,1)$ de $\mathbb{R}^{2}$ et c'est la dynamique dans ce disque qui nous intéresse. Dans toute la suite nous considérons la restriction de $\varphi_{\vartheta}$ au disque fermé $\overline{\mathbb{D}(0,1)}$ en gardant la même notation $\varphi_{\vartheta}$. On vérifie sans peine que tous les $\varphi_{\left.\vartheta\right|_{\mathbb{S}^{1}}}: \mathbb{S}^{1} \rightarrow \mathbb{S}^{1}$ sont conjugués à l'application $z \mapsto z^{2}$ $\mathrm{du}$ cercle dans lui-même. Le diamètre $[-1,1]$ va sur le diamètre $\left[-\mathrm{e}^{\mathrm{i} \vartheta}, \mathrm{e}^{\mathrm{i} \vartheta}\right]$ et est en particulier invariant lorsque $\vartheta=0$. Le diamètre vertical $x_{1}=0$ est contracté sur le point $-\mathrm{e}^{\mathrm{i} \vartheta}$. Ainsi un point de type $\left(0, x_{2}\right)$ va à la première itération sur $-\mathrm{e}^{\mathrm{i} \vartheta}$ puis reste par itération sur le cercle $\mathbb{S}^{1}$ où, génériquement sur $\vartheta$, son orbite est dense. Remarquons que les courbes « algébriques $\gg$ réelles $\left(\varphi^{k}\right)^{-1}([-\mathrm{i}, \mathrm{i}])$ présentent une propriété analogue après $k+1$ itérations. La description de $\varphi_{0}:\left(x_{1}, x_{2}\right) \mapsto\left(2 x_{1}^{2}-\right.$ $\left.1,2 x_{1} x_{2}\right)$ est relativement raisonnable. Sur le cercle unité $\varphi_{0}$ coïncide avec $x \mapsto x^{2}$ et est induite par $\mathrm{M} \mapsto \mathrm{M}^{2}$ restreinte à $\mathbb{H}_{1}$. On se souvient que $\mathcal{O}\left(\mathrm{M} ; \Phi_{\mathrm{Id}}\right)$ est 
tracée dans le 2-plan engendré par $\mathrm{M}$, Id et $-\mathrm{Id}$. En se restreignant à $\mathbb{H}_{1}$ nous en déduisons que les orbites de $\Phi_{{\mathrm{Id} \mid \mathbb{H}_{1}}_{1}}$ sont tracées sur les cercles de $\mathbb{S}^{3}$ obtenus comme intersection du 2-plan, réel cette fois, passant par M, Id et -Id (excepté lorsque M est Id ou $-\mathrm{Id})$. Les orbites de $\varphi_{0}$ sont donc tracées sur les projections (par $(x, y) \mapsto(x, 0))$ de ces cercles; ce sont les ellipses passant par les points $(1,0)$ et $(-1,0)$ et tangentes au cercle unité en $(1,0)$ et $(-1,0)$ :

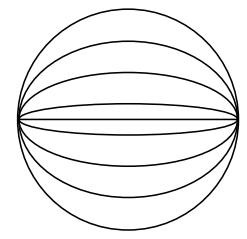

On vérifie facilement que la restriction de $\varphi_{0}$ à chaque ellipse est conjuguée à $x \mapsto x^{2}$, sauf évidemment dans le cas spécial où l'ellipse dégénère sur l'intervalle $[-1,1]$. Dans ce cas on constate que $\varphi_{\left.0\right|_{[-1,1]}}$ est l'application $x_{1} \mapsto 2 x_{1}^{2}-1$ qui n'est rien d'autre que la célèbre application « logistique $\gg$ à conjugaison près (4]). En fait comme nous l'avons dit l'application $\Phi_{\text {Id }}$ est compatible à la conjugaison. Sur $\mathbb{S}^{3} \simeq \mathbb{H}^{1}$ les classes de conjugaison sont déterminées par la trace $x+\bar{x}=2 x_{1}$ et sont donc des 2 -sphères $\left(x_{1}=\right.$ cte $) \cap \mathbb{S}^{3}$. Par exemple si $\mathrm{M} \in \mathbb{H}_{1}$ est périodique pour $\varphi_{\mathrm{Id}}$ alors toute la sphère $\mathbb{S}^{2}=$ classe de $\mathrm{M}$ est formée d'éléments périodiques ce que l'on peut voir directement sur l'application $\varphi_{0}$; celle-ci respecte les droites verticales dans leur ensemble :

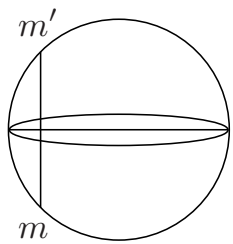

On peut déduire d'ailleurs de façon directe la dynamique de l'application logistique (qui se fait usuellement de façon combinatoire via un codage RLC, voir (4) de la dynamique de l'application du cercle $x \mapsto x^{2}$ (qui elle se fait en utilisant l'écriture diadique des angles). Si on s'intéresse aux points périodiques de période 2 de $\varphi_{0}$ on trouve (outre le point $1 \sim(1,0)$ ) le segment $\left[\mathrm{j}, \mathrm{j}^{2}\right]$. La restriction de $\varphi_{0}$ à $\left[\mathrm{j}, \mathrm{j}^{2}\right]$ est donnée par $\left(-\frac{1}{2}, x_{2}\right) \mapsto\left(-\frac{1}{2},-x_{2}\right)$ qui est évidemment une involution. Son point fixe $\left(-\frac{1}{2}, 0\right)$ correspond au point fixe $-\frac{1}{2}$ de l'application logistique. Remarquons que l'ensemble des points périodiques de $\varphi_{0}$ est une union dense de segments verticaux $\left[m, m^{\prime}\right]$, les segments passant par les points périodiques de $x \mapsto x^{2}$. Comme $\varphi_{\pi}$ est conjugué à $\varphi_{0}$ par $\left(x_{1}, x_{2}\right) \mapsto\left(-x_{1},-x_{2}\right)$ tout ce que nous avons dit pour $\varphi_{0}$ s'adapte via la conjugaison à $\varphi_{\pi}$. Nous conjecturons que pour les applications $\varphi_{\vartheta}$ avec $\vartheta$ générique les points périodiques de période donnée sont en nombre fini à l'inverse de $\varphi_{0}$. Par exemple l'application

$$
\varphi_{\pi / 2}:\left(x_{1}, x_{2}\right) \mapsto\left(-2 x_{1} x_{2}, 2 x_{1}^{2}-1\right)
$$

a les points fixes suivants $(0,-1),\left(\frac{1}{2},-\frac{1}{2}\right),\left(-\frac{1}{2},-\frac{1}{2}\right)$ et ses points 2-périodiques sont en nombre fini contrairement à ceux de $\varphi_{0}$. En effet $\varphi_{\pi / 2}^{2}:\left(x_{1}, x_{2}\right) \mapsto$ 
$\left(4 x_{1} x_{2}\left(2 x_{1}^{2}-1\right), 8 x_{1}^{2} x_{2}^{2}-1\right)$ a pour points fixes les trois points qui précèdent auxquels s'ajoutent $\left(\frac{\sqrt{3}}{2},-\frac{1}{2}\right)\left(-\frac{\sqrt{3}}{2}, \frac{1}{2}\right)$ qui sont les deux racines 3ième de l'unité $\mathrm{j}$ et $j^{2}$.

Nous avons essayé de préciser les points 2-périodiques et leurs bifurcations en utilisant la technique des bases de Grobner qui fonctionne bien lorsque

$\Delta(\vartheta)=\left(4 \cos ^{2} \vartheta-3\right)\left(2 \cos ^{3} \vartheta-3 \cos ^{2} \vartheta+2\right)\left(2 \cos ^{3} \vartheta+3 \cos ^{2} \vartheta-2\right)(\cos \vartheta-1)(\cos \vartheta+1)$

est non nulle. Sous cette hypothèse $(\Delta(\vartheta) \neq 0)$ l'application $\varphi_{\vartheta}$ a sept points périodiques de période 2 , tous réels, i.e. dans $\mathbb{R}^{2}$, qui sont les suivants :

(a) le point fixe $\mathrm{e}^{-\mathrm{i} \vartheta} \simeq(\cos \vartheta,-\sin \vartheta)$;

(b) les deux points fixes

$$
\text { (b.1) }\left(-\frac{1}{2}, \frac{\cos \vartheta-1}{2 \sin \vartheta}\right) \quad \text { et } \quad(\mathfrak{b} .2)\left(\frac{1}{2},-\frac{\cos \vartheta+1}{2 \sin \vartheta}\right) \text {; }
$$

(c) les deux points 2-périodiques de la restriction $\varphi_{\vartheta_{\mathbb{S}^{1}}}: z \mapsto \mathrm{e}^{\mathrm{i} \vartheta} z^{2}$ qui sont je $\mathrm{j}^{-\mathrm{i} \vartheta}$ et $\mathrm{j}^{2} \mathrm{e}^{-\mathrm{i} \vartheta}$

( $\mathfrak{d})$ deux autres points 2-périodiques donnés par

$$
\begin{aligned}
& \text { (d.1) }\left(\frac{\sqrt{1+4 \cos ^{2} \vartheta}-1}{4 \cos \vartheta}, \frac{\sin \vartheta\left(\sqrt{1+4 \cos ^{2} \vartheta}+1\right)}{4 \cos ^{2} \vartheta}\right), \\
& \text { (d.2) }\left(-\frac{\sqrt{1+4 \cos ^{2} \vartheta}+1}{4 \cos \vartheta}, \frac{\sin \vartheta\left(1-\sqrt{1+4 \cos ^{2} \vartheta}\right)}{4 \cos ^{2} \vartheta}\right) .
\end{aligned}
$$

Remarquons que lorsque $\vartheta$ tend vers 0 , les points fixes de type $(\mathfrak{b})$ tendent respectivement vers $\left(-\frac{1}{2}, 0\right)$ qui est point fixe de $\varphi_{0}$ et vers $\ll\left(\frac{1}{2}, \infty\right) \gg$. Lorsque $\vartheta$ tend vers $\pi$ le phénomène inverse se produit : un point fixe tend vers $\left(\frac{1}{2}, 0\right)$ (point fixe de $\varphi_{\pi}$ ) et l'autre s'échappe.

Un point de type (b.1), resp. (b.2) est dans le disque unité fermé si et seulement si $-\frac{2 \pi}{3} \leq \vartheta \leq \frac{2 \pi}{3}$, resp. si et seulement si $\frac{\pi}{3} \leq \vartheta \leq \frac{5 \pi}{3}$. Notons que les points fixes de $\varphi_{\vartheta}$ ne sont jamais contractants. Les points 2-périodiques de type $(\mathfrak{d})$ sont dans le disque unité fermé si et seulement si $-\frac{\pi}{4} \leq \vartheta \leq \frac{\pi}{4}$ ou $\frac{3 \pi}{4} \leq \vartheta \leq \frac{5 \pi}{4}$ et ils le sont simultanément.

Nous avons appliqué la procédure type JuLIA évoquée précédemment aux applications $\varphi_{\vartheta}$ dans le disque unité en choisissant une donnée de contrôle $\rho$ plus petite que 1 mais très voisine de 1 . Les figures suivantes mesurent donc la façon dont les orbites s'approchent du bord du disque et à quelle vitesse. Par exemple dans la première figure où nous appliquons la procédure à $\varphi_{0}$, les points périodiques non situés sur le bord ne s'en approchent pas ce qui produit les lignes verticales « indigo $\sim$ rouge $\gg$. Remarquons dans ce cas spécial la liaison avec le problème suivant : pour la transformation du cercle $z \mapsto z^{2}$ étudier comment les orbites approchent le point fixe 1 et à quelle vitesse. Ceci explique la structure cantorique que l'on observe sur cette première figure.

Nous avons choisi de présenter des figures pour $\kappa$ petit pour la qualité visuelle; on constate expérimentalement qu'elles ne varient pas qualitativement quand $\kappa$ augmente. Le paramètre $r$, resp. $\rho$ prend la valeur 1 , resp. $\sqrt{0.99}$. 


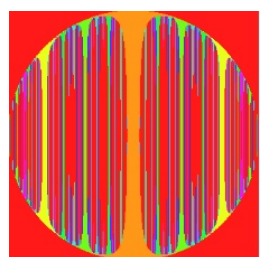

$\vartheta=0$

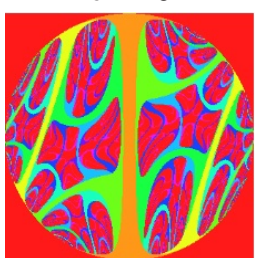

$\vartheta=0.6$

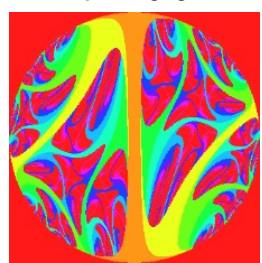

$\vartheta=1.3$

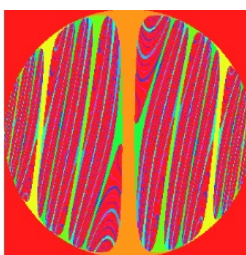

$\vartheta=0.2$

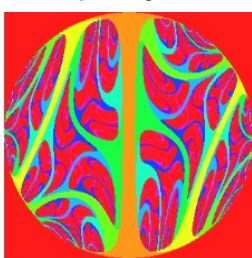

$\vartheta=0.7$

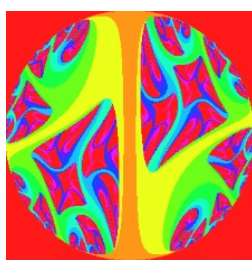

$\vartheta=1.4$

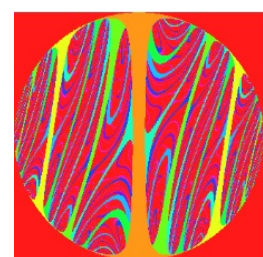

$\vartheta=0.3$

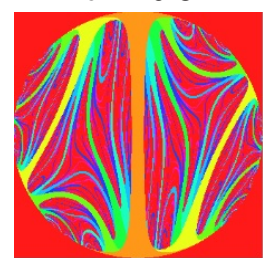

$\vartheta=0.8$

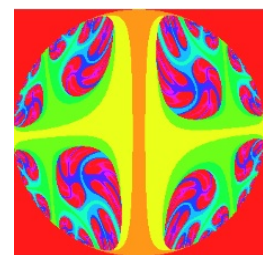

$\vartheta=1.5$

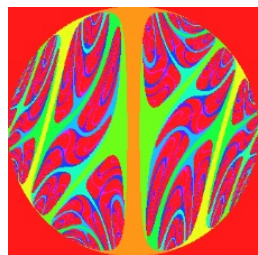

$\vartheta=0.5$

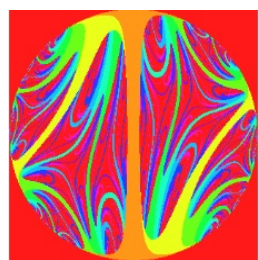

$\vartheta=1$

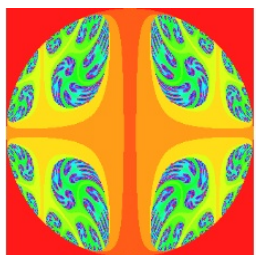

$\vartheta=\frac{\pi}{2}$

Le tracé ponctuel des orbites ne s'avère pas très probant. Pour pallier ce défaut nous avons itéré des segments verticaux par les applications $\varphi_{\vartheta}$. Nous présentons ci-dessous les onze premiers itérés de la droite $\mathcal{D}$ d'équation $x_{1}=0.6$ intersectée avec le disque de rayon 1 par l'application $\varphi_{\pi / 2}$.
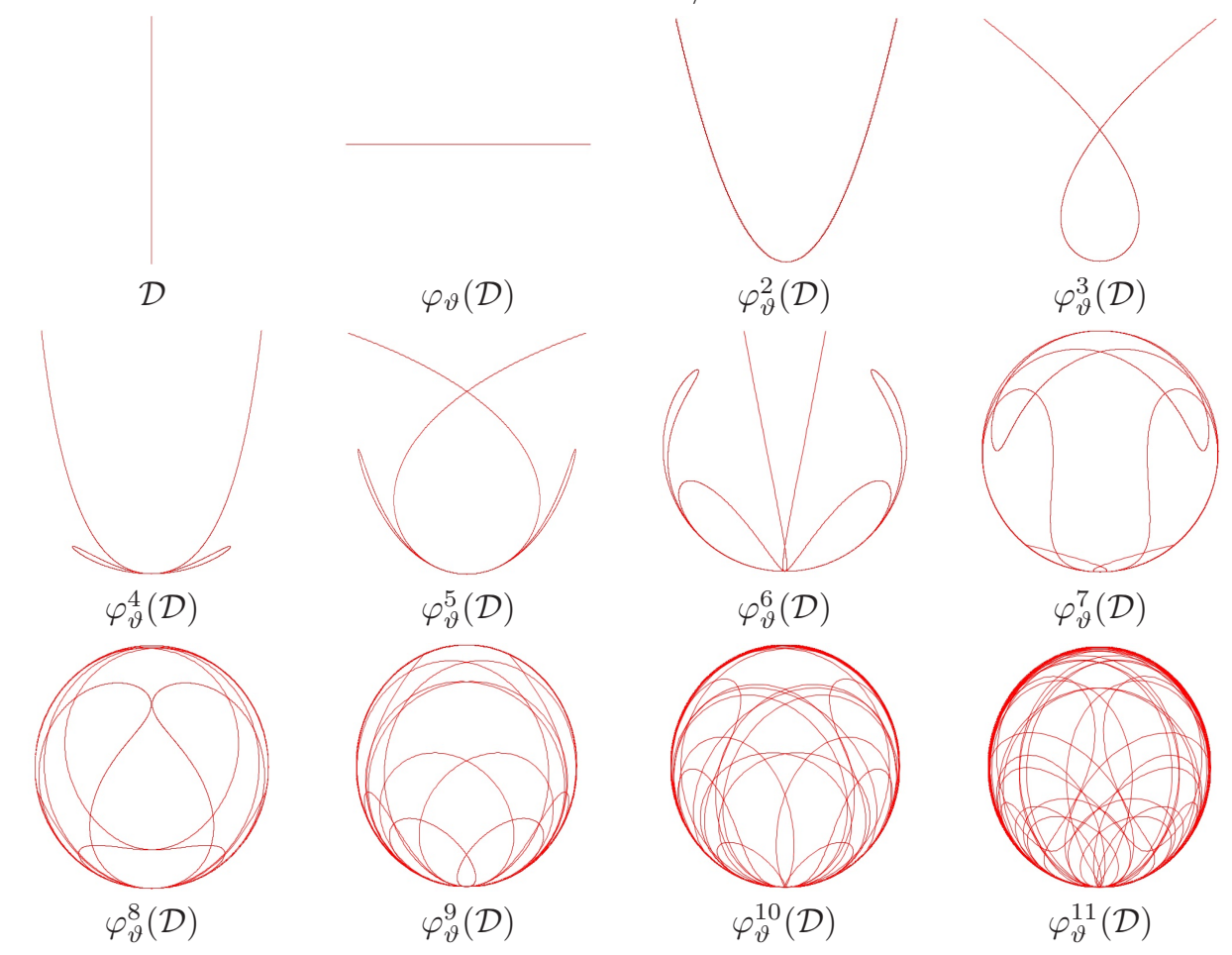
On constate que rapidement les courbes images s'accumulent sur le cercle du bord tout entier.

Problèmes 6. (1) Pour $\mathrm{A}=\operatorname{diag}\left(\lambda, \frac{1}{\lambda}\right)$ caractériser en fonction de $\lambda$ les orbites qui sont bornées en particulier celles qui ne sont pas dans $\mathrm{W}_{\mathrm{A}}^{s}(\mathbf{0})$.

(2) Caractériser le bassin d'attraction $\mathrm{W}_{\mathrm{A}}^{s}(\mathbf{0})$ de l'origine et si possible décrire son bord.

(3) Donner la description précise de la dynamique des $\Phi_{\mathrm{A}}$ dans le cas quaternionique.

4.1.7. Centralisateur. Comme nous l'avons fait au paragraphe précédent nous allons déterminer le groupe $\operatorname{Aut}\left(\mathcal{M}(2 ; \mathbb{C}) ; \Phi_{\mathrm{A}}\right)$ pour $\mathrm{A}=\operatorname{diag}\left(\lambda, \frac{1}{\lambda}\right), \lambda^{2} \neq 1$.

Proposition 4.5. Soit A une matrice de la forme $\operatorname{diag}\left(\lambda, \frac{1}{\lambda}\right)$ avec $\lambda^{2} \neq 1$. Le groupe $\operatorname{Aut}\left(\mathcal{M}(2 ; \mathbb{C}) ; \Phi_{\mathrm{A}}\right)$ est engendré par les $\sigma_{\mathrm{P}}$ avec $\mathrm{P}$ diagonale; en fait $\operatorname{Aut}\left(\mathcal{M}(2 ; \mathbb{C}) ; \Phi_{\mathrm{A}}\right)$ s'identifie à $\mathbb{C}^{*}$ agissant sur $\mathcal{M}(2 ; \mathbb{C})$ de la façon suivante : $(x, y, z, t, \alpha) \mapsto\left(x, \alpha y, \frac{z}{\alpha}, t\right)$. Les orbites de cette action sont aussi celles du champ de vecteurs invariant $y \frac{\partial}{\partial y}-z \frac{\partial}{\partial z}$.

Démonstration. Avec des arguments analogues à ceux utilisés dans la démonstration de la Proposition 3.8 on montre qu'un élément $\varphi$ de $\operatorname{Aut}\left(\mathcal{M}(2 ; \mathbb{C}) ; \Phi_{\mathrm{A}}\right)$ est nécessairement linéaire.

Écrivons $\varphi$ sous la forme $\left(\ell_{1}, \ell_{2}, \ell_{3}, \ell_{4}\right)$ les $\ell_{i}$ désignant des formes linéaires. La fibration $y / z=$ cte est invariante par $\Phi_{\mathrm{A}}$, plus précisément nous avons $\frac{y}{z} \circ \Phi_{\mathrm{A}}=\lambda^{2} \frac{y}{z}$. Nous en déduisons l'égalité $\ell_{3}\left(\ell_{2} \circ \Phi_{\mathrm{A}}\right)=\lambda^{2}\left(\ell_{3} \circ \Phi_{\mathrm{A}}\right) \ell_{2}$. Ceci implique, puisque les seuls 3-plans invariants par $\Phi_{\mathrm{A}}$ sont $y=0$ et $z=0$, l'alternative suivante :

$$
\text { ou bien } \ell_{2}=\alpha y, \ell_{3}=\beta z, \quad \text { ou bien } \ell_{2}=\alpha z, \ell_{3}=\beta y \text {. }
$$

En réécrivant $\frac{\ell_{2}}{\ell_{3}} \circ \Phi_{\mathrm{A}}=\lambda^{2} \frac{\ell_{2}}{\ell_{3}}$ on constate que la seconde éventualité n'arrive que si $\lambda^{4}=1$.

Dans le premier cas la commutation de $\varphi$ et $\Phi_{\mathrm{A}}$ entraîne que $\varphi=\left(x, \alpha y, \frac{z}{\alpha}, t\right)$. Lorsque $\lambda^{4}=1, \lambda$ vaut i ou $-\mathrm{i}$ (les valeurs propres de A sont supposées distinctes). Écrivons $\ell_{1}$ (resp. $\ell_{4}$ ) sous la forme $a_{1} x+b_{1} y+c_{1} z+d_{1} t$ (resp. $\left.a_{4} x+b_{4} y+c_{4} z+d_{4} t\right)$. L'égalité $\Phi_{\mathrm{A}} \varphi=\varphi \Phi_{\mathrm{A}}$ conduit à

$$
b_{1}=c_{1}=b_{4}=c_{4}=a_{1} d_{1}=a_{4} d_{4}=0
$$

et

$$
\begin{gathered}
a_{4}=-1-a_{1}, \quad d_{4}=-1-d_{1}, \quad a_{1}^{2}=a_{1}, \quad d_{1}^{2} \lambda^{2}=d_{1}, \\
\lambda \alpha \beta=\frac{d_{1}}{\lambda}+a_{1}, \quad a_{4}^{2}=\lambda^{2} a_{4}, \quad a_{4}+\frac{d_{4}}{\lambda}=\frac{\alpha \beta}{\lambda} .
\end{gathered}
$$

Un calcul montre que si $a_{1}$ est nul, alors $\lambda=-1$, sinon $d_{1}=0$ et $\lambda=1$. Ces deux cas sont exclus par l'hypothèse $\lambda^{2} \neq 1$.

4.2. Cas non diagonalisable. Considérons les applications de la forme $\mathrm{M} \mapsto \mathrm{AM}^{2}$ avec A inversible non diagonalisable. Nous nous ramenons après conjugaison ad-hoc à $\mathrm{A}=\left[\begin{array}{ll}1 & 1 \\ 0 & 1\end{array}\right]$; par suite

$$
\Phi_{\mathrm{A}}\left(\left[\begin{array}{cc}
x & y \\
z & t
\end{array}\right]\right)=\left[\begin{array}{cc}
\left(x^{2}+y z\right)+z(x+t) & y(x+t)+\left(t^{2}+y z\right) \\
z(x+t) & t^{2}+y z
\end{array}\right] .
$$


La quadrique de dimension 2 formée des matrices nilpotentes est toujours envoyée sur 0 par $\Phi_{\mathrm{A}}$ et $\mathfrak{s l}(2 ; \mathbb{C})$ est encore contractée, cette fois sur $\mathbb{C} A$. La fibration $\frac{x-t}{z}=$ cte est invariante par $\Phi_{\mathrm{A}}$; la seule fibre invariante est $z=0$. Le feuilletage $z \frac{\partial}{\partial x}+(t-x) \frac{\partial}{\partial y}-z \frac{\partial}{\partial t}$ est invariant par $\Phi_{\mathrm{A}}$. On constate que l'ensemble des matrices non inversibles est invariant par $\Phi_{\mathrm{A}}$, les 2-plans $x=z=0, y=t=0, z=$ $t=0$ aussi. Toute matrice de la forme $\left[\begin{array}{cc}\lambda & \mu \\ 0 & \lambda\end{array}\right]$ commute à $\mathrm{A}$; par conséquent $\left\{\left[\begin{array}{ll}\lambda & \mu \\ 0 & \lambda\end{array}\right] \mid \lambda, \mu \in \mathbb{C}\right\}$ est invariant par multiplication par A et par $\Phi_{\mathrm{A}}$.

Comme au 4.1.7 on démontre l'énoncé suivant.

Proposition 4.6. Soit A la matrice $\left[\begin{array}{ll}1 & 1 \\ 0 & 1\end{array}\right]$. Le groupe $\operatorname{Aut}\left(\mathcal{M}(2 ; \mathbb{C}) ; \Phi_{\mathrm{A}}\right)$ est engendré par les $\sigma_{\mathrm{P}}$ où $\mathrm{P}$ commute à $\mathrm{A}$.

4.2.1. Étude des points fixes et périodiques. On peut vérifier que les points fixes de $\Phi_{\mathrm{A}}$ sont

$$
\text { 0, } \quad\left[\begin{array}{cc}
1 & -1 \\
0 & 1
\end{array}\right], \quad\left\{\left[\begin{array}{ll}
1 & y \\
0 & 0
\end{array}\right] \mid y \in \mathbb{C}\right\} \text {. }
$$

D'après ce qui précède les points périodiques de $\Phi_{\mathrm{A}}$ sont contenus dans l'hyperplan $z=0$. Un calcul montre que

$$
\Phi_{\mathrm{A}}^{n}\left(\left[\begin{array}{ll}
x & y \\
0 & t
\end{array}\right]\right)=\left[\begin{array}{cc}
x^{2^{n}} & \left(\prod_{i=0}^{n-1}\left(x^{2^{i}}+t^{2^{i}}\right)\right) y+t^{2^{n}}+\sum_{k=1}^{n-1}\left(t^{2^{k}} \prod_{i=k}^{n-1}\left(x^{2^{i}}+t^{2^{i}}\right)\right) \\
0 & t^{2^{n}}
\end{array}\right] .
$$

Si $(\underline{x}, \underline{y}, \underline{z}, \underline{t})$ est un point périodique de $\Phi_{\mathrm{A}}$ de période $n$, alors $\underline{z}=0$ et

(a) ou bien $\underline{x}=\underline{t}=0$;

(b) ou bien $\underline{x}^{2^{n}-1}=1$ et $\underline{t}=0$;

(c) ou bien $\underline{x}=0$ et $\underline{t}^{2^{n}-1}=1$;

(d) ou bien $\underline{x}^{2^{n}-1}=\underline{t}^{2^{n}-1}=1$.

Examinons ces éventualités au cas par cas.

(a) Si $\underline{x}=\underline{t}=0$ alors nécessairement $y=0$, i.e. $\mathbf{0}$ est périodique.

(b) Si $\underline{x}^{2^{n}-1}=1$ et $\underline{t}=0$, on constate que $(\underline{x}, y, 0,0), \underline{x}^{2^{n}-1}=1$, est périodique de période $n$.

(c) Si $\underline{x}=0$ et $\underline{t}^{2^{n}-1}=1$, alors $\Phi_{\mathrm{A}}^{n}(0, \underline{y}, 0, \underline{t})=(0, \underline{y}+n \underline{t}, 0, \underline{t})$.

(d) Plaçons-nous maintenant dans le second cas : $\underline{x}^{\overline{2}^{n}-1}=\underline{t}^{2^{n}-1}=1$. Nous allons raisonner suivant que $\underline{t}=\underline{x}$ et $\underline{t} \neq \underline{x}$.

Dans un premier temps supposons que $\underline{t}=\underline{x}$ auquel cas $\Phi_{\mathrm{A}}^{n}(\underline{x}, y, 0, \underline{x})=\left(\underline{x}^{2^{n}}, 2^{n} y\right.$ $\left.+\left(2^{n}-1\right) \underline{x}, 0, \underline{x}^{2^{n}}\right)$; ces considérations produisent les points périodiques suivants $\bigcup_{n \geq 0}\left\{(\underline{x},-\underline{x}, 0, \underline{x}) \mid \underline{x}^{2^{n}-1}=1\right\}$.

Reste l'éventualité $\underline{x} \neq \underline{t}$. Posons

$$
B_{n}(\underline{x}, \underline{t})=\prod_{i=0}^{n-1}\left(\underline{x}^{2^{i}}+\underline{t}^{2^{i}}\right), \quad C_{n}(\underline{x}, \underline{t})=\underline{t}^{2^{n}}+\sum_{k=1}^{n-1} t^{2^{k}}\left(\prod_{i=k}^{n-1}\left(\underline{x}^{2^{i}}+\underline{t}^{2^{i}}\right)\right) .
$$


Sous toutes ces notations et hypothèses nous avons

$$
\Phi_{\mathrm{A}}=\left(\underline{x}^{2^{n}}, B_{n}(\underline{x}, \underline{t}) y+C_{n}(\underline{x}, \underline{t}), 0, \underline{t}^{2^{n}}\right), \quad \underline{x}^{2^{n}-1}=\underline{t}^{2^{n}-1}=1, \quad \underline{x} \neq \underline{t} .
$$

Nous sommes donc ramenés à considérer l'équation $B_{n}(\underline{x}, \underline{t}) y+C_{n}(\underline{x}, \underline{t})=y$ où $\underline{x}$, $\underline{t}$ sont des racines $\left(2^{n}-1\right)$-ième de l'unité distinctes. On peut vérifier que $B_{n}(\underline{x}, \underline{t})=$ 1. Si pour chaque couple $(\underline{x}, \underline{t})$ de racines $\left(2^{n}-1\right)$-ième de l'unité distinctes $C_{n}(\underline{x}, \underline{t})$ est non nul, il n'y a pas de point périodique de période $n$ de la forme $(\underline{x}, y, 0, \underline{t})$ avec $\underline{x} \neq \underline{t}, \underline{x}^{2^{n}-1}=\underline{t}^{2^{n}-1}=1$; sinon, pour tous les $(\underline{x}, \underline{t})$ tels que

$$
\underline{x}^{2^{n}-1}=\underline{t}^{2^{n}-1}=1, \quad \underline{x} \neq \underline{t}, \quad C_{n}(\underline{x}, \underline{t})=0,
$$

les $(\underline{x}, y, 0, \underline{t})$ sont des points périodiques de période $n$.

Notons qu'il arrive que $C_{n}(\underline{x}, \underline{t})$ soit non nul, par exemple lorsque $n=2, \underline{t}=\mathrm{j}$ et $\underline{x}=1$.

4.2.2. Étude de quelques orbites non périodiques. Comme précédemment nous avons $\operatorname{det} \Phi_{\mathrm{A}}(\mathrm{M})=(\operatorname{det} \mathrm{M})^{2}$ et $\operatorname{donc} \operatorname{det} \Phi_{\mathrm{A}}^{k}(\mathrm{M})=(\operatorname{det} \mathrm{M})^{2^{k}}$. Ainsi, pour tout $\mathrm{M}$ satisfaisant $|\operatorname{det} \mathrm{M}|>1$, nous avons $\lim _{k \rightarrow+\infty}\left\|\Phi_{\mathrm{A}}^{k}(\mathrm{M})\right\|=+\infty$.

Comme dans le cas diagonal, si $\mathrm{M}$ appartient au polydisque $\Delta(\rho)$, alors $\left\|\Phi_{\mathrm{A}}(\mathrm{M})\right\|$ $\leq 2 \rho^{2}$ et $\left\|\Phi_{\mathrm{A}}^{k}(\mathrm{M})\right\| \leq 2^{2^{k}-1} \rho^{2^{k}}$ qui entraîne l'inclusion

$$
\Delta\left(\frac{1}{2}\right) \subset \mathrm{W}_{\mathrm{A}}^{s}(\mathbf{0}) \text {. }
$$

Donnons quelques exemples d'orbites bornées. Remarquons que $\Phi_{\mathrm{A}}^{n}(x, y, 0, x)=$ $x^{2^{n}-1}\left(x,\left(2^{n}-1\right) x+2^{n} y, 0, x\right)$; par suite, dès que $\mathrm{M}$ est de la forme

$$
\begin{gathered}
{\left[\begin{array}{cc}
x & -x \\
0 & x
\end{array}\right] \text { avec }|x|<1 \text { ou }} \\
{\left[\begin{array}{cc}
x & y \\
0 & x
\end{array}\right] \text { avec }|x|<1 \text { et }\left(2^{n}-1\right) x+2^{n} y=0 \text { pour un certain } n}
\end{gathered}
$$

l'orbite de M est bornée.

Puisque $\Phi_{\mathrm{A}}^{n}(0, y, 0, t)=t^{2^{n}-1}(0, n t+y, 0, t)$, l'orbite de $\left[\begin{array}{ll}0 & y \\ 0 & t\end{array}\right]$, avec $|t|<1$ et $y=-n t$ pour un certain entier $n$, est bornée.

Étant donné que $\Phi_{\mathrm{A}}^{n}(x, y, 0,0)=x^{2^{n}-1}(x, y, 0,0)$, toute matrice $\left[\begin{array}{ll}x & y \\ 0 & 0\end{array}\right]$ avec $|x|<1$ a une orbite bornée.

Problèmes 7. (1) Décrire les points périodiques de l'application $\Phi_{\mathrm{A}}$ et leur adhérence.

(2) Décrire le bassin d'attraction $\mathrm{W}_{\mathrm{A}}^{s}(\mathbf{0})$ et son bord.

Remerciements. Nous remercions M. BAKer, D. Boucher, S. CAntat, G. Casale, S. Gouëzel et F. Loray pour leur disponibilité.

\section{REFERENCES}

[1] E. Bedford and J. Smillie. Fatou-Bieberbach domains arising from polynomial automorphisms. Indiana Univ. Math. J., 40(2):789-792, 1991. MR:1119197(92k:32044)

[2] F. Berteloot and J. J. Lob. Holomorphic equivalence between basins of attraction in $\mathbf{C}^{2}$. Indiana Univ. Math. J., 45(2):583-589, 1996. MR1414343(97f:32037)

[3] D. Cerveau and A. Lins Neto. Hypersurfaces exceptionnelles des endomorphismes de CP $(n)$. Bol. Soc. Brasil. Mat. (N.S.), 31(2):155-161, 2000. MR1785266 (2002h:32014) 
[4] P. Collet and J.-P. Eckmann. Iterated maps on the interval as dynamical systems. Modern Birkhäuser Classics. Birkhäuser Boston Inc., Boston, MA, 2009. Reprint of the 1980 edition. MR2541754(2010j:37060)

[5] G. Fischer. Complex analytic geometry. Lecture Notes in Mathematics, Vol. 538. SpringerVerlag, Berlin, 1976. MR0430286 (55:3291)

[6] S. Friedland and J. Milnor. Dynamical properties of plane polynomial automorphisms. Ergodic Theory Dynam. Systems, 9(1):67-99, 1989. MR991490 (90f:58163)

[7] R. Galeeva and A. Verjovsky. Quaternion dynamics and fractals in $\mathbf{R}^{4}$. Internat. J. Bifur. Chaos Appl. Sci. Engrg., 9(9):1771-1775, 1999. Discrete dynamical systems. MR 1728736 (2000g:37052)

[8] B. Malgrange. Frobenius avec singularités. II. Le cas général. Invent. Math., 39(1):67-89, 1977. MR0508170(58:22685b)

[9] J. Milnor. Pasting together Julia sets: a worked out example of mating. Experiment. Math., 13(1):55-92, 2004. MR2065568 (2005c:37087)

Membre de l'Institut Universitaire de France. IRMAR, UMR 6625 du CNRS, UniverSité de Rennes 1, 35042 Rennes, France

E-mail address: dominique.cerveau@univ-rennes1.fr

Institut de Mathématiques de Jussieu, Université Paris 7, Projet Géométrie et Dynamique, Site Chevaleret, Case 7012, 75205 Paris Cedex 13, France

E-mail address: deserti@math.jussieu.fr

Current address: Universität Basel, Mathematisches Institut, Rheinsprung 21, CH-4051, Basel, Switzerland 